\title{
On Bar Recursion and Choice in a Classical Setting
}

\author{
Valentin Blot and Colin Riba \\ ENS de Lyon, Université de Lyon, LIP * \\ valentin.blot@ens-lyon.fr http://perso.ens-lyon.fr/valentin.blot/ \\ colin.riba@ens-lyon.fr http://perso.ens-lyon.fr/colin.riba/
}

\begin{abstract}
We show how Modified Bar-Recursion, a variant of Spector's Bar-Recursion due to Berger and Oliva can be used to realize the Axiom of Choice in Parigot's Lambda-Mu-calculus, a direct-style language for the representation and evaluation of classical proofs.

We rely on Hyland-Ong innocent games. They provide a model to perform the usual infinitary reasoning on Bar-Recursion needed for the realization of classical choice, and where, moreover, the standard datatype of natural numbers is in the image of a CPS-translation.
\end{abstract}

\section{Introduction}

Peano's Arithmetic in all finite types $\left(\mathrm{PA}^{\omega}\right)$ is a multisorted version of firstorder Peano's Arithmetic, with one sort for each simple type, together with the constants of Gödel's System T and their defining equations. When augmenting $\mathrm{PA}^{\omega}$ with the Axiom of Choice (CAC), we obtain a system known to contain large parts of classical analysis (see e.g. [9, 16]). A similar system can be obtained by extending Peano's Arithmetic to Second-Order Logic (see e.g. [16]).

We are interested here in the realizability interpretation of PA ${ }^{\omega}+$ CAC. Realizability is a mathematical tool, part of the Curry-Howard correspondence, used to extract computational content from formal proofs.

The usual route to get a computational interpretation of (some extension of) $\mathrm{PA}^{\omega}$ is to apply a negative translation, yielding proofs in (some extension of) Heyting's Arithmetic in all finite types ( $\mathrm{HA}^{\omega}$, the intuitionist variant of $\mathrm{PA}{ }^{\omega}$, see e.g. [19]), followed by a computational interpretation of the translated proofs. Realizability for $\mathrm{HA}^{\omega}$ can be obtained in simply-typed settings, typically using Gödel's System T. In this way, CAC is translated to a formula which can be realized by combining a realizer of the Intuitionistic Axiom of Choice (IAC) with a realizer of the Double Negation Shift (DNS, see Sect. 3). Intuitionistic choice is easily realizable, and realizers of DNS can be obtained by adapting Spector's Bar-Recursion to realizability [3, 4].

We are interested here in a computational interpretation of $\mathrm{PA}^{\omega}+\mathrm{CAC}$ based on a realizability interpretation directly for classical proofs. It has been noted by Griffin [6] that the control operator call/cc of the functional language Scheme

* UMR 5668 CNRS ENS Lyon UCBL INRIA 
can be typed using Peirce's Law, which gives full Classical Logic when added to Intuitionistic Logic. Since then, there have been much work on calculi for Classical Logic, starting from Parigot's $\lambda \mu$-calculus [14]. Moreover, Krivine has developed a notion of Classical Realizability for Second-Order Peano's Arithmetic which relies on Girard's System F [10] (see also [13, 12]).

In this paper, we investigate a version of Spector's Bar-Recursion in a classical realizability setting for $\mathrm{PA}^{\omega}$, obtained by adapting Krivine's Realizability to a simply-typed extension of Parigot's $\lambda \mu$-calculus. Our main point concerns Bar-Recursion. Handling Bar-Recursion in realizability (typically to show that it realizes DNS) involves some reasoning on infinite non-constructive objects. This infinitary reasoning can be made directly at the level of a programming language extended with infinite terms, as in [3]. Another possibility, as done in [4], is to internalize realizability in the logic, and reason within the logic on finite terms under some axioms (typically bar-induction) which can only be validated in an infinitary model (typically a model of PCF). Similarly to [3] and contrary to [4], our notion of realizability is not internalized in the logic. For extraction of programs from proofs, our approach is similar to [4]: we separate the programming language, which is kept finitary, from the infinitary model in which the realizability argument is made.

It is well-known that most non-degenerate models and operational semantics for the $\lambda \mu$-calculus rely on CPS translations. We work here with the call-by-name translation of Lafont-Reus-Streicher (see e.g. [18, 15]). However, a difficulty arises with Bar-Recursion: the CPS translation makes its types seemingly too high to conclude with the usual argument [4,3]. A solution is given by the innocent unbracketed Hyland-Ong game model of $\mathrm{PCF}[8,11]$ : the usual flat game arena of natural numbers can be built as a CPS translation in the coproduct completion of the model (this was observed, but not exploited, in [11]).

We define a notion of classical realizability in this game model. Our main result is that the usual realizer of classical choice obtained by combining a realizer of IAC with Berger-Oliva's variant of Bar-Recursion [4], is indeed a realizer of classical choice in our framework. We then obtain an extraction result for the $\lambda \mu$-terms by a logical relation argument (see e.g. [2]), relating the operational semantics and the model.

The paper is organized as follows: We begin by presenting PA ${ }^{\omega}$ in Sect. 2. We then briefly discuss the usual computational interpretation of CAC by negative translation in Sect. 3. In Sect. 4, we present the bare minimum we need on Hyland-Ong games. Parigot's $\lambda \mu$-calculus, as well as its game interpretation and its operational semantics are discussed in Sect. 5. We then devise our notion of realizability in Sect. 6 and discuss the realization of CAC in Sect. 7. Detailed proofs are given in Appendices.

\section{Peano's Arithmetic in All Finite Types}

In this section, we briefly discuss the logical system on which we work in this paper, namely PA ${ }^{\omega}$ (Peano's Arithmetic in all finite types), as well as its extension 
with the axiom of choice. We build on usual versions of $\mathrm{HA}^{\omega}$ (see e.g. [19, 9]), with ideas of $[14,10]$ for classical logic.

Language. The language of $\mathrm{PA}^{\omega}$ is multisorted, with one sort for each simple type. We use the following syntax of simple types, where $\iota$ is intended to be the base type of natural numbers:

$$
\sigma, \tau \in \mathcal{T} \quad::=\quad \iota \quad|\quad \sigma \rightarrow \tau| \sigma \times \tau
$$

We assume given, for each simple type $\tau$, a countable set $\mathcal{V}_{\tau}=\left\{x^{\tau}, y^{\tau}, \ldots\right\}$ of individual variables of type $\tau$. Individuals are simply-typed terms

$$
a, b \in \mathcal{I} \quad::=x^{\tau}|a b \quad| \quad \text { с }
$$

where $(a b)^{\tau}$ provided $a^{\sigma \rightarrow \tau}, b^{\sigma}$ for some $\sigma$, and c ranges over the constants $0^{\iota}, \mathrm{S}^{\iota \rightarrow \iota}, \operatorname{Rec}^{\tau \rightarrow(\iota \rightarrow \tau \rightarrow \tau) \rightarrow \iota \rightarrow \tau}$, Pair ${ }^{\sigma \rightarrow \tau \rightarrow \sigma \times \tau}, \mathrm{P}_{i}^{\tau_{1} \times \tau_{2} \rightarrow \tau_{i}} \quad(i=1,2), \mathrm{k}^{\sigma \rightarrow \tau \rightarrow \sigma}$ and $\mathbf{s}^{(\rho \rightarrow \sigma \rightarrow \tau) \rightarrow(\rho \rightarrow \sigma) \rightarrow \rho \rightarrow \tau}$. Let $\mathcal{I}_{0}$ be the set of closed individuals and $\mathcal{I}_{0}^{\tau}$ be the set of closed individuals of type $\tau$.

Formulas are defined as follows:

$$
A, B \in \mathcal{F} \quad::=\left(a^{\tau} \neq_{\tau} b^{\tau}\right) \quad|\quad \perp \quad| \quad A \Rightarrow B \quad|\quad A \wedge B \quad| \forall x^{\tau} A
$$

Note the atomic inequality $\left({ }_{-} \neq_{\tau}-\right)$. It is inspired from Krivine's work [10] and will greatly eases our realizability interpretation (see Sect. 6).

We use the following abbreviations:

$$
\begin{aligned}
& \neg A \quad:=A \Rightarrow \perp \quad \exists x^{\tau} A \quad:=\neg \forall x^{\tau} \neg A \\
& \left(a={ }_{\tau} b\right) \quad:=\neg\left(a \neq_{\tau} b\right) \quad A \vee B \quad:=\quad \neg(\neg A \wedge \neg B)
\end{aligned}
$$

Deduction. We consider the following deduction system, parametrized by a set $A x$ of axioms (containing only closed formulas).

$$
\begin{gathered}
\frac{\Gamma, A \vdash A \mid \Delta}{\Gamma, A \vdash B \mid \Delta} \frac{\Gamma \vdash \perp \mid \Delta}{\Gamma \vdash A \Rightarrow B \mid \Delta \quad} \quad \frac{\Gamma \vdash A x) \quad \frac{\Gamma \vdash A}{\Gamma \vdash a^{\tau} \neq_{\tau} b^{\tau} \mid \Delta}}{\Gamma \vdash B \mid \Delta} \\
\frac{\Gamma \vdash A \Rightarrow B \mid \Delta}{\Gamma \vdash A|\Delta \quad \Gamma \vdash B| \Delta} \frac{\frac{\Gamma \vdash A_{1} \wedge A_{2} \mid \Delta}{\Gamma \vdash A_{i} \mid \Delta}(i=1,2)}{\Gamma \vdash A \wedge B \mid \Delta} \quad \frac{\Gamma \vdash \forall x^{\tau} A \mid \Delta}{\Gamma \vdash A\left[a^{\tau} / x\right] \mid \Delta} \\
\frac{\Gamma \vdash A \mid \Delta}{\Gamma \vdash \forall x^{\tau} A \mid \Delta}(x \notin \mathrm{FV}(\Gamma, \Delta)) \quad \frac{(\Gamma \vdash \Delta, A)}{\Gamma \vdash A \mid \Delta}
\end{gathered}
$$


This system is chosen so as to have a direct extraction of realizers in Parigot's $\lambda \mu$-calculus (see Sect. 5 and 6 ).

Note that the Ex Falso rule is restricted to atomic formulas. For each formula $A$ one can easily derive $\Gamma \vdash A \mid \Delta$ from $\Gamma \vdash \perp \mid \Delta$. The introduction rules for existential quantification and disjunction are easy to derive:

$$
\frac{\Gamma \vdash A \mid \Delta}{\Gamma \vdash A \vee B \mid \Delta} \quad \frac{\Gamma \vdash A\left[a^{\tau} / x\right] \mid \Delta}{\Gamma \vdash \exists x^{\tau} A \mid \Delta}
$$

Moreover, one can derive Peirce's Law and Double Negation Elimination:

$$
\overline{\Gamma \vdash((A \Rightarrow B) \Rightarrow A) \Rightarrow A \mid \Delta} \quad \overline{\Gamma \vdash((A \Rightarrow \perp) \Rightarrow \perp) \Rightarrow A \mid \Delta}
$$

as well as the elimination rules of disjunction and existential quantification: $\Gamma \vdash C \mid \Delta$ provided $\Gamma \vdash A \vee B|\Delta, \Gamma, A \vdash C| \Delta$ and $\Gamma, B \vdash C \mid \Delta$; and $\Gamma \vdash C \mid \Delta$ provided $\Gamma \vdash \exists x^{\tau} A \mid \Delta$ and $\Gamma, A \vdash C \mid \Delta$ with $x$ not free in $\Gamma, C, \Delta$.

Axioms for Equality and Arithmetic. The axioms of $\mathrm{PA}^{\omega}$ are the universal closures of the following formulas:

- Equality axioms are reflexivity $\forall x^{\tau}\left(x={ }_{\tau} x\right)$ and Leibniz's scheme:

$$
\text { for all formula } A, \quad \forall x^{\tau} y^{\tau}\left(A[x / z] \Rightarrow \neg A[y / z] \Rightarrow x \neq_{\tau} y\right)
$$

Note that the usual version of Leibniz's scheme is derivable:

$$
\forall x^{\tau} y^{\tau}\left(x={ }_{\tau} y \Rightarrow A[x / z] \Rightarrow A[y / z]\right)
$$

- Equational axioms (with variables of the appropriate types):

$$
\begin{gathered}
\mathrm{k} x y={ }_{\tau} x \quad \mathrm{~s} x y z={ }_{\tau} x z(y z) \quad \mathrm{P}_{i}\left(\operatorname{Pair} x_{1} x_{2}\right)=_{\tau_{i}} x_{i} \quad(i=1,2) \\
\operatorname{Rec} x y 0={ }_{\tau} x \quad \operatorname{Rec} x y(\mathrm{~S} z)=_{\tau} y z(\operatorname{Rec} x y z)
\end{gathered}
$$

- Arithmetic axioms are $\forall x^{\iota}(\mathrm{S} x \neq \iota 0)$ and the Induction scheme:

$$
\text { for all formula } A, \quad A[0 / x] \Rightarrow \forall x^{\iota}(A \Rightarrow A[\mathrm{~S} x / x]) \Rightarrow \forall x^{\iota} A
$$

We write $\mathrm{PA}^{\omega} \vdash A$ if $\vdash A \mid$ is derivable using the axioms of $\mathrm{PA}^{\omega}$.

Axiom of Choice. Given $\tau \in \mathcal{T}$, we write CAC $^{\iota, \tau}$ for the following version of the axiom (scheme) of choice:

$$
\text { for all formula } A, \quad\left(\forall x^{\iota} \exists y^{\tau} A\right) \Rightarrow \exists f^{\iota \rightarrow \tau} \forall x^{\iota} A[f x / y]
$$

Note that this unfolds to

$$
\forall x^{\iota}\left(\forall y^{\tau}(A \Rightarrow \perp) \Rightarrow \perp\right) \Rightarrow \forall f^{\iota \rightarrow \tau}\left(\forall x^{\iota} A[f x / y] \Rightarrow \perp\right) \Rightarrow \perp
$$

We write $\mathrm{PA}^{\omega}+\mathrm{CAC}^{\iota,-}$ for provability in $\mathrm{PA}^{\omega}$ using any $\mathrm{CAC}^{\iota, \tau}$ for $\tau \in \mathcal{T}$. 


\section{Intuitionistic Modified Realizability and Bar-Recursion}

In this section, we briefly and informally recall the realization of CAC via negative translation to $\mathrm{HA}^{\omega}+$ DNS, and discuss some aspects of our realization of CAC.

$\mathrm{HA}^{\omega}$ can be obtained from our presentation of $\mathrm{PA}^{\omega}$ by restricting deduction to intuitionistic sequents, i.e. sequents of the form $\Gamma \vdash A \mid$. One also has to take a primitive notion of equality (instead of our primitive $\left({ }_{-} \neq_{\tau_{-}}\right)$), and primitive existential quantification (disjunction can be coded). Gödel's negative translation can be adapted from $\mathrm{PA}^{\omega}$ to $\mathrm{HA}^{\omega}$ : let $\left.\left({ }_{-}\right)\right\urcorner$commute over the connectives of $\mathrm{PA}^{\omega}$ (remember that there is no $\vee, \exists$ in $\mathcal{F}$ ), and put $\neg \neg$ in front of atomic formulas, after having replaced $\left(a \neq_{\tau} b\right)$ by $\neg\left(a=_{\tau} b\right)$. It is equivalent to leave $\perp$ unchanged and map $\left(a \neq_{\tau} b\right)$ to $\neg\left(a=_{\tau} b\right)$.

Let us briefly discuss Modified Realizability. To each closed formula $A$ is associated a simple type $A^{*}$ of potential realizers of $A$. Actual realizers of $A$ are closed terms of type $A^{*}$ satisfying a property, usually written $t \Vdash A$, defined by induction on $A$. Typical clauses are:

$$
\begin{aligned}
& t^{\iota} \Vdash \perp:=\perp \quad t^{\iota} \Vdash\left(a={ }_{\tau} b\right):=\left(a={ }_{\tau} b\right) \\
& t \Vdash(A \Rightarrow B):=\forall u(u \Vdash A \Rightarrow t u \Vdash B) \quad t \Vdash \forall x^{\tau} A:=\forall x^{\tau}(t x \Vdash A) \\
& t \Vdash(A \wedge B):=\left(\mathrm{P}_{1} t \Vdash A \wedge \mathrm{P}_{2} t \Vdash B\right) \quad t \Vdash \exists x^{\tau} A:=\left(\mathrm{P}_{2} t \Vdash A\left[\mathrm{P}_{1} t / x\right]\right)
\end{aligned}
$$

Note that this provides a realizer, written $t_{\mathrm{IAC}}$, of intuitionistic choice $\left(\mathrm{IAC}^{\sigma, \tau}\right)^{1}$ :

$$
\lambda z . \operatorname{Pair}\left(\lambda x . \mathrm{P}_{1}(z x)\right)\left(\lambda x . \mathrm{P}_{2}(z x)\right) \quad \Vdash \quad\left(\forall x^{\sigma} \exists y^{\tau} A\right) \Rightarrow \exists f^{\sigma \rightarrow \tau} \forall x^{\sigma} A[f x / y]
$$

A proof in $\mathrm{PA}^{\omega}$ of a formula $A$ can be mapped to a realizer of the negative translation $A^{\urcorner}$of $A^{2}$. For CAC $C^{\iota, \tau}$, this leads (modulo the intuitionistic equivalence $\neg \forall \neg \longleftrightarrow \neg \neg \exists$ ) to find a realizer of

$$
\left.\left.\forall x^{\iota} \neg \neg \exists y^{\tau} A\right\urcorner \Rightarrow \neg \neg \exists f^{\iota \rightarrow \tau} \forall x^{\iota} A\right\urcorner[f x / y]
$$

It is well-known (see e.g. [3, 4, 9]) that such a realizer can be obtained by combining a realizer of $\mathrm{IAC}^{\iota, \tau}$ with a realizer of the Double Negation Shift

$$
\left(\forall x^{\iota} \neg \neg B\right) \Rightarrow \neg \neg \forall x^{\iota} B
$$

for the instance $B:=\exists y^{\tau} A$. Assuming $\Psi$ realizes this instance of DNS, we get

$$
\left.\lambda z . \lambda k . \Psi z\left(\lambda a . k\left(t_{\mathrm{IAC}} a\right)\right) \quad \Vdash \quad \forall x^{\iota} \neg \neg \exists y^{\tau} A \Rightarrow \Rightarrow \neg \neg \exists f^{\iota \rightarrow \tau} \forall x^{\iota} A\right\urcorner[f x / y]
$$

The reader can check that we obtain the following realizer of CAC:

$$
\begin{aligned}
t_{\mathrm{CAC}}:=\quad \lambda z \cdot \lambda c \cdot \Psi\left(t_{\neg \neg \exists} z\right)\left(\lambda a . c\left(\lambda x \cdot \mathrm{P}_{1}(a x)\right)\left(\lambda x \cdot \mathrm{P}_{2}(a x)\right) \quad \Vdash\right. \\
\forall x^{\iota}\left(\forall y^{\tau}(A \Rightarrow \perp) \Rightarrow \perp\right) \Rightarrow \forall f^{\iota \rightarrow \tau}\left(\forall x^{\iota} A[f x / y] \Rightarrow \perp\right) \Rightarrow \perp
\end{aligned}
$$

$\overline{1}$ We use the $\lambda$-notation for individual terms in $\mathcal{I}$.

${ }^{2}$ To get extraction for $\Pi_{2}^{0}$-formulas, one can adapt Friedman's trick by defining $\left(t^{\iota} \Vdash\right.$ $\perp)$ as $\Perp(t)$, where $\Perp$ is a given predicate, see e.g. [4] and also Sect. 6 . 
with $t_{\neg \neg \exists}:=\lambda a . \lambda x \cdot \lambda k . a x(\lambda y \cdot \lambda z . k($ Pair $y z)) \Vdash \forall x^{\iota} \neg \forall y^{\tau} \neg A \Rightarrow \forall x^{\iota} \neg \neg \exists y^{\tau} A$

Realizers $\Psi$ of DNS can be obtained by adapting Spector's Bar-Recursion to realizability $[3,4]$.

In this paper, we show that (the interpretation in a suitable model of) $t_{\text {CAC }}$ realizes $\mathrm{CAC}^{\iota, \tau}$, for a notion of realizability defined for (the interpretation in a suitable model of) an extension of Parigot's $\lambda \mu$-calculus [14]. We actually show that Berger and Oliva's version of Bar-Recursion [4] realizes a version of CAC with partially relativized quantifiers (see Sect. 6 and 7). For PA ${ }^{\omega}$, the realizers we obtain seem to translate to the same realizers as obtained by a negative translation from $\mathrm{PA}^{\omega}$ to $\mathrm{HA}^{\omega}$ followed by Friedman's translation and a realizability interpretation, as devised at the beginning of this section. However, a difficulty arises with Bar-Recursion: if we directly CPS-translate Bar-Recursion, we obtain a term of type

$$
(\iota\urcorner \rightarrow(\tau \rightarrow \iota\urcorner) \rightarrow \iota\urcorner) \rightarrow((\iota\urcorner \rightarrow \tau) \rightarrow \iota\urcorner) \rightarrow \iota\urcorner
$$

where $\iota\urcorner:=(\iota \rightarrow \iota) \rightarrow \iota$. But the types seem too high to conclude using the usual argument $[4,3]$.

A solution is to perform a CPS translation such that $\iota\urcorner$ is not too different from a type of natural numbers in a model of PCF. A natural candidate would be something like

$$
\iota\urcorner \quad:=(\iota \rightarrow \mathrm{R}) \rightarrow \mathrm{R}
$$

Obvious choices for $\mathrm{R}$ (e.g. a one-point object) tend to give degenerated results: typically, in domains (and even predomains [18]), taking $R=\{\perp\}(R=\emptyset)$ gives a unique inhabitant in $\iota\urcorner$.

We show that this can be solved by using the coproduct completion (given by the Fam construction, see e.g. [1]) of Hyland-Ong innocent unbracketed games for PCF. We rely on the fact (observed, but not exploited, in [11]) that in the coproduct completion of these games, the basic type of natural numbers is of the form $(\llbracket \mathbb{N} \rrbracket \rightarrow R) \rightarrow R$, for the one-move game $R$ and the countable family of empty games $\llbracket \mathbb{N} \rrbracket$ (see Sect. 4 ).

\section{The Model of Hyland-Ong Games}

In this section, we present the bare minimum we need on Hyland-Ong games. We use innocent unbracketed games, combined with the coproduct completion provided by the Fam construction. Details can be found in e.g. [8, 7, 11, 1].

\subsection{Arenas and Strategies}

Definition 4.1 (Arena). An arena is a countable forest of moves. Each move is given a polarity $O$ (for Opponent) or $P$ (for Player or Proponent):

- A root is of polarity $O$.

- A move which is not a root has the inverse polarity of that of his parent. 
A root of an arena is also called an initial move. We will often identify an arena with its set of moves.

Definition 4.2 (Justified sequence). Given an arena $\mathcal{A}$, we define a justified sequence on $\mathcal{A}$ to be a finite word $s$ on $\mathcal{A}$ together with a partial justifying function $f:|s| \rightarrow|s|$ such that:

- If $f(i)$ is undefined, then $s_{i}$ is an initial move.

- If $f(i)$ is defined, then $f(i)<i$ and $s_{i}$ is a child of $s_{f(i)}$.

We denote the empty justified sequence by $\epsilon$. Remark here that by definition of the polarity, if $f(i)$ is undefined ( $s_{i}$ is initial), then $s_{i}$ is of polarity $O$, and if $f(i)$ is defined, then $s_{i}$ and $s_{f(i)}$ are of opposite polarities. Also, $f(0)$ is never defined, and so $s_{0}$ is always an initial $O$-move. A justified sequence is represented for example as:

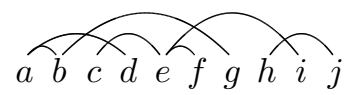

If $\mathcal{A}$ is an arena, $X$ is a subset of $\mathcal{A}$ and $s$ is a justified sequence on $\mathcal{A}$, then $s_{\mid X}$ is the subsequence of $s$ consisting of the moves of $s$ which are in $X$.

Definition 4.3 (Play). $A$ play $s$ on $\mathcal{A}$ is an even and alternating justified sequence of $\mathcal{A}$, i.e., for any $i, s_{2 i}$ is a $O$-move and $s_{2 i+1}$ is a $P$-move. We denote the set of plays of $\mathcal{A}$ by $\mathcal{P}_{\mathcal{A}}$.

A play on an arena is the trace of an interaction between a program and a context, each one performing an action alternatively.

Definition 4.4 (Strategy). A strategy $\sigma$ on $\mathcal{A}$ is a non-empty even-prefixclosed set of finite plays on $\mathcal{A}$ such that:

- $\sigma$ is deterministic

- $\sigma$ is innocent

The definitions of determinism and innocence are standard and can be found for example in $[7,8]$.

Cartesian Closed Structure. The constructions we use will sometimes contain multiple copies of the same arena (for example $\mathcal{A} \rightarrow \mathcal{A}$ ), so we distinguish the instances with superscripts (for example $\mathcal{A}^{(1)} \rightarrow \mathcal{A}^{(2)}$ ).

Let $\mathcal{U}$ be the empty arena and $\mathcal{V}$ be the arena with only one (opponent) move. If $\mathcal{A}$ and $\mathcal{B}$ are arenas consisting of the trees $\mathcal{A}_{1} \ldots \mathcal{A}_{p}$ and $\mathcal{B}_{1} \ldots \mathcal{B}_{q}$, then the arenas $\mathcal{A} \rightarrow \mathcal{B}$ and $\mathcal{A} \times \mathcal{B}$ can be represented as follows:

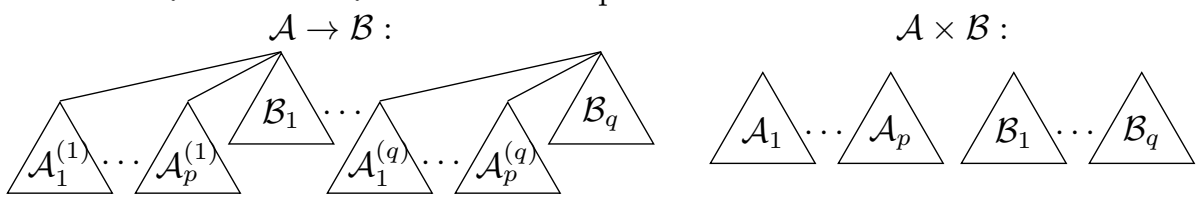

The constructions described here define a cartesian closed category whose objects are arenas and morphisms are innocent strategies. Details of the construction can be found in $[7,8]$. In the following this category will be denoted as $\mathcal{G}$. 


\subsection{The Fam Construction}

Our model is built as a continuation category [18]. In order to make explicit the double negation translation of the base types, we base the model on the category of continuations $\mathrm{R}^{\mathrm{Fam}(\mathcal{G})}$, where $\operatorname{Fam}(\mathcal{G})$ is a variant of the coproduct completion described in [1] applied to the category $\mathcal{G}$ defined in Sect. 4.1.

Definition $4.5(\operatorname{Fam}(\mathcal{G}))$. The objects of $\operatorname{Fam}(\mathcal{G})$ are families of objects of $\mathcal{G}$ indexed by at most countable sets, and a morphism from $\left\{A_{i} \mid i \in I\right\}$ to $\left\{B_{j} \mid j \in\right.$ $J\}$ is a function $f: I \rightarrow J$ together with a family of morphisms of $\mathcal{G}$ from $A_{i}$ to $B_{f(i)}$, for $i \in I$.

See [5] for details on the differences with [1]. Note that $\operatorname{Fam}(\mathcal{G})$ is a distributive category with finite products and coproducts, and has exponentials of all singleton families. The empty product and terminal object is the singleton family $\{\mathcal{U}\}$, the empty sum and initial object is the empty family \{\} , and:

$$
\begin{aligned}
\left\{A_{i} \mid i \in I\right\} \times\left\{B_{j} \mid j \in J\right\} & :=\left\{A_{i} \times B_{j} \mid(i, j) \in I \times J\right\} \\
\left\{A_{i} \mid i \in I\right\}+\left\{B_{j} \mid j \in J\right\} & :=\left\{C_{k} \mid k \in I \uplus J\right\} \text { where } C_{k}:=\left\{\begin{array}{l}
A_{k} \text { if } k \in I \\
B_{k} \text { if } k \in J
\end{array}\right. \\
\left\{B_{0}\right\}\left\{A_{i} \mid i \in I\right\} & :=\left\{\Pi_{i \in I} B_{0}^{A_{i}}\right\}
\end{aligned}
$$

We fix once and for all:

$$
\mathrm{R}:=\{\mathcal{V}\}
$$

which is an object of $\operatorname{Fam}(\mathcal{G})$ as a singleton family. $\mathrm{R}$ has all exponentials as stated above. Note that the canonical morphism $\delta_{A}: A \rightarrow \mathrm{R}^{\left(\mathrm{R}^{A}\right)}$ is a mono.

The category of continuations $\mathrm{R}^{\mathrm{Fam}(\mathcal{G})}$ is the full subcategory of $\operatorname{Fam}(\mathcal{G})$ consisting of the objects of the form $\mathrm{R}^{A}$. The objects of $\mathrm{R}^{\mathrm{Fam}(\mathcal{G})}$ are singleton families, and $\mathrm{R}^{\mathrm{Fam}(\mathcal{G})}$ is isomorphic to $\mathcal{G}$. We will consider that objects and morphisms of $\mathrm{R}^{\mathrm{Fam}(\mathcal{G})}$ are arenas and strategies and we will use the vocabulary defined at the end of Sect. 4.1 on $\mathrm{R}^{\mathrm{Fam}(\mathcal{G})}$ also.

\subsection{The Type Structure}

We use the lambda notation in $\mathrm{R}^{\mathrm{Fam}(\mathcal{G})}$, i.e. we build simply-typed $\lambda$-terms with constants in $\mathrm{R}^{\mathrm{Fam}(\mathcal{G})}$. We write them using bold symbols (such as $\boldsymbol{\lambda},\left\langle_{-},{ }_{-}\right\rangle$etc) in order make no confusion with the syntactic $\lambda \mu$-terms of Section 5 .

Interpretation of Simple Types. Let $\llbracket \mathbb{N} \rrbracket$ be the object $\left\{\mathcal{U}_{n} \mid n \in \mathbb{N}\right\}$ of $\operatorname{Fam}(\mathcal{G})$. We use the interpretation of simple types proposed in [18] (see also [15]). Given a simple type $\tau \in \mathcal{T}$, we associate two objects of $\mathrm{R}^{\mathrm{Fam}(\mathcal{G})}$ : the object $[\tau]$ of programs of type $\tau$, and the object $\llbracket \tau \rrbracket$ of continuations of type $\tau$. We let

$$
\llbracket \iota \rrbracket:=\mathrm{R}^{\llbracket \mathbb{N} \rrbracket} \quad \llbracket \sigma \rightarrow \tau \rrbracket:=\mathrm{R}^{\llbracket \sigma \rrbracket} \times \llbracket \tau \rrbracket \quad \llbracket \sigma \times \tau \rrbracket:=\llbracket \sigma \rrbracket+\llbracket \tau \rrbracket \quad[\tau]:=\mathrm{R}^{\llbracket \tau \rrbracket}
$$

Note that $\llbracket \sigma \rightarrow \tau \rrbracket=[\sigma] \times \llbracket \tau \rrbracket$, and moreover

$$
[\sigma \rightarrow \tau] \quad=\quad \mathrm{R}^{[\sigma] \times \llbracket \tau \rrbracket} \simeq \mathrm{R}^{\llbracket \tau \rrbracket^{\mathrm{R}^{\llbracket \sigma \rrbracket}}} \quad \text { and } \quad[\sigma \times \tau] \quad \simeq \quad \mathrm{R}^{\llbracket \sigma \rrbracket} \times \mathrm{R}^{\llbracket \tau \rrbracket}
$$


Representation of Arithmetic Constants. In $\operatorname{Fam}(\mathcal{G})$ a morphism from the terminal object $\{\mathcal{U}\}$ to $\llbracket \mathbb{N} \rrbracket=\left\{\mathcal{U}_{n} \mid n \in \mathbb{N}\right\}$ is given by a function from the singleton set to $\mathbb{N}$ together with a strategy from $\mathcal{U}$ to $\mathcal{U}$. Since there is only one such strategy, such a morphism is given by a natural number. We will call this morphism $\widetilde{n}$. Similarly a morphism from $\llbracket \mathbb{N} \rrbracket$ to $\llbracket \mathbb{N} \rrbracket$ is given by a function from $\mathbb{N}$ to $\mathbb{N}$. This leads to a morphism $\widetilde{\text { succ }}: \llbracket \mathbb{N} \rrbracket \rightarrow \llbracket \mathbb{N} \rrbracket$ for the successor function on $\llbracket \mathbb{N} \rrbracket$.

Moreover, given $a:[\tau]$ (officially, $a:\{\mathcal{U}\} \rightarrow[\tau]$ in $\operatorname{Fam}(\mathcal{G})$ ), and $b: \llbracket \mathbb{N} \rrbracket \rightarrow$ $[\tau] \rightarrow[\tau]$, we can define by induction on $n \in \mathbb{N}$ a morphism $\widetilde{r}_{a, b}: \llbracket \mathbb{N} \rrbracket \rightarrow[\tau]$ such that $\widetilde{r}_{a, b} \widetilde{0}=a$ and $\widetilde{r}_{a, b}(\widetilde{n+1})=b \widetilde{n}\left(\widetilde{r}_{a, b}(\widetilde{n})\right)$. This leads to $\widetilde{\text { rec }}:=\boldsymbol{\lambda} a \cdot \boldsymbol{\lambda} b \cdot \widetilde{r}_{a, b}$ in $[\tau] \rightarrow(\llbracket \mathbb{N} \rrbracket \rightarrow[\tau] \rightarrow[\tau]) \rightarrow \llbracket \mathbb{N} \rrbracket \rightarrow[\tau]$

We now discuss the object of $\mathrm{R}^{\mathrm{Fam}(\mathcal{G})}$ associated to the base type $\iota$. We have:

$$
[\iota]:=\mathrm{R}^{\mathrm{R}^{\mathbb{N} \rrbracket}}=\mathrm{R}^{\mathrm{R}^{\left\{\mathcal{U}_{n} \mid n \in \mathbb{N}\right\}}} \simeq \mathrm{R}^{\Pi_{n \in \mathbb{N} R}} \simeq\left\{\mathcal{V}^{\Pi_{n \in \mathbb{N}} \mathcal{V}}\right\}
$$

Note that this is the usual flat arena of natural numbers:

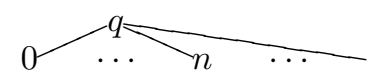

It is easy to see that $\lambda k . k \tilde{n}$ corresponds to the strategy answering $n$ to the initial opponent question $q$. Moreover, the only inhabitants of $[\iota]$ are the empty strategy $\perp_{[\iota]}$ and the strategies $\lambda k . k \tilde{n}$ for $n \in \mathbb{N}$.

The arithmetical constants of System $\mathrm{T}$ will be interpreted in $\mathrm{R}^{\mathrm{Fam}(\mathcal{G})} \mathrm{using}$ succ $:[\iota] \rightarrow[\iota]$ defined as succ $:=\boldsymbol{\lambda} n \cdot \boldsymbol{\lambda} k \cdot n(\boldsymbol{\lambda} x \cdot k(\widetilde{\operatorname{succ}} x))$ and rec $:[\tau] \rightarrow[\iota \rightarrow$ $\tau \rightarrow \tau] \rightarrow[\iota] \rightarrow[\tau]$ with rec := $\boldsymbol{\lambda} u \cdot \boldsymbol{\lambda} v \cdot \boldsymbol{\lambda} n \cdot \boldsymbol{\lambda} k \cdot n\left(\boldsymbol{\lambda} x \cdot \widetilde{\operatorname{rec}} u\left(\boldsymbol{\lambda} y \cdot v^{\bullet}(\boldsymbol{\lambda} k . k y)\right) x k\right)$, where $v^{\bullet}:=\boldsymbol{\lambda} x \cdot \boldsymbol{\lambda} y \cdot \boldsymbol{\lambda} z . v\langle x, y, z\rangle$ (see [5] for details).

It is convenient to use the notations $\left(_{-}\right)^{\bullet}$ and $\left(_{-}\right)^{\circ}$ for resp. curryfication and uncurryfication. Note that as with $v^{\bullet}$ above, the amount to which an expression is curryfied/uncurryfied depends on the context, and moreover that in $\mathcal{G},\left(_{-}\right)^{\bullet}$ and $\left({ }_{-}\right)^{\circ}$ are the identity.

\section{Lambda-Mu-Calculus}

We present here an extension of Parigot's $\lambda \mu$-calculus [14] that we will use as a programming language for our realizers. We begin by a basic language, which essentially adds pairs and products to the original calculus. We then present an extension with the arithmetic constants of Gödel's System T, which will be used for the realization of PA ${ }^{\omega}$. Finally, we discuss the interpretation, along the lines of $[15]$, of the calculus in the model $\mathrm{R}^{\mathrm{Fam}(\mathcal{G})}$, and present an operational semantics using an abstract machine adapted from [18].

Syntax and Typing. We assume given two countable sets $\operatorname{Var}=\{x, y, z, \ldots\}$ and $\mathrm{CVar}=\{\alpha, \beta, \gamma, \ldots\}$ of respectively term and continuation variables. The $\lambda \mu$-terms are defined as follows:

$t, u \in \Lambda \quad:=x \mid=x$. $\quad x \quad \mid \begin{array}{lllllllll} & t u & \mid \mu \alpha . v & \langle t, u\rangle & \mathrm{p}_{1}(t) & \mathrm{p}_{2}(t)\end{array}$ where $v$ is a named term: $\quad v \quad::=[\alpha] t$ 
They are typed by extending Parigot's system [14] with rules for product types:

$$
\begin{gathered}
\frac{\Gamma \vdash t: \tau \mid \Delta, \alpha: \tau}{\Gamma, x: \tau \vdash x: \tau \mid \Delta} \quad \frac{v:(\Gamma \vdash \Delta, \alpha: \tau)}{[\alpha] t:(\Gamma \vdash \Delta, \alpha: \tau)} \quad \frac{\Gamma \vdash \mu \alpha \cdot v: \tau \mid \Delta}{\Gamma \vdash \mu} \\
\frac{\Gamma, x: \tau \vdash t: \sigma \mid \Delta}{\Gamma \vdash \lambda x . t: \tau \rightarrow \sigma \mid \Delta} \quad \frac{\Gamma \vdash t: \sigma \rightarrow \tau|\Delta \quad \Gamma \vdash u: \sigma| \Delta}{\Gamma \vdash t u: \tau \mid \Delta} \\
\frac{\Gamma \vdash t: \tau|\Delta \quad \Gamma \vdash u: \sigma| \Delta}{\Gamma \vdash\langle t, u\rangle: \tau \times \sigma \mid \Delta} \quad \frac{\Gamma \vdash t: \tau_{1} \times \tau_{2} \mid \Delta}{\Gamma \vdash \mathrm{p}_{i}(t): \tau_{i} \mid \Delta}(i=1,2)
\end{gathered}
$$

Extension with Arithmetic Constants. We write $\Lambda_{T}$ for the set of $\lambda \mu$-terms obtained by extending the grammar of $\Lambda$ with the following productions:

$$
t, u \quad::=\ldots|\bar{n}| \operatorname{succ} \mid \operatorname{rec}(t, u)
$$

where $n \in \mathbb{N}$. We extend the typing rules of $\Lambda$ with the following ones:

$$
\overline{\Gamma \vdash \bar{n}: \iota \mid \Delta} \quad \overline{\Gamma \vdash \operatorname{succ}: \iota \rightarrow \iota \mid \Delta} \quad \frac{\Gamma \vdash t: \tau|\Delta \quad \Gamma \vdash u: \iota \rightarrow \tau \rightarrow \tau| \Delta}{\Gamma \vdash \operatorname{rec}(t, u): \iota \rightarrow \tau \mid \Delta}
$$

Interpretation in $\mathrm{R}^{\mathrm{Fam}(\mathcal{G})}$. The interpretation of $\Lambda_{T}$ in $\mathrm{R}^{\mathrm{Fam}(\mathcal{G})}$ follows the lines of [15]. A term $\vdash t: \tau \mid$ is interpreted by $[t] \in[\tau]$. To make the presentation simpler, we use $\boldsymbol{\lambda}$-expressions in $\mathrm{R}^{\mathrm{Fam}(\mathcal{G})}$ build from the variables of $\Lambda_{T}$ with the following convention: a term variable $x$ of type $\tau$ (resp. a continuation variable $\alpha$ of type $\sigma$ ) in $\Lambda_{T}$ becomes a variable $x$ of type [ $\left.\tau\right]$ (resp. a variable $\alpha$ of type $\llbracket \sigma \rrbracket)$ in the $\boldsymbol{\lambda}$-calculus of $\mathrm{R}^{\mathrm{Fam}(\mathcal{G})}$ :

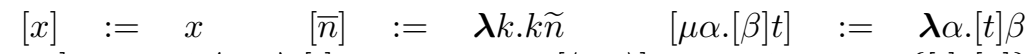

$$
\begin{aligned}
& {[\lambda x . t]:=\lambda\langle x, k\rangle \cdot[t] k \quad[\langle t, u\rangle] \quad:=\lambda k \text {.case } k\{[t],[u]\}} \\
& {[t u]:=\lambda k \cdot[t]\langle[u], k\rangle \quad\left[\mathrm{p}_{i}(t)\right]:=\boldsymbol{\lambda} k \cdot[t]\left(\mathbf{i n}_{i} k\right)} \\
& {[\text { succ }] \quad:=\lambda\langle n, k\rangle \text {. succ } n k \quad[\operatorname{rec}(t, u)]:=\quad \lambda\langle n, k\rangle \cdot \operatorname{rec}[t][u] n k}
\end{aligned}
$$

Operational Semantics. We now present an operational semantics for $\Lambda_{T}$ using an abstract machine. The machine is derived from the interpretation of $\Lambda_{T}$ in $\mathrm{R}^{\mathrm{Fam}(\mathcal{G})}$, following the method of [18]. Our machine is actually an adaptation of the machine of [18] to a typed language with arithmetic constants.

The machine evaluates triples of the form $(t, e, \pi)$, where $t$ is a $\lambda \mu$-term, $e$ is an environment and $\pi$ is a stack. Environments map term variables to closures and continuation variables to stacks. Stacks, closures and environments are defined by mutual induction as usual:

$$
\begin{aligned}
& \text { Env. } \quad e \in \mathrm{E} \quad::=\varepsilon|(x, c):: e \quad| \quad(\alpha, \pi):: e \\
& \text { Closures } \quad c \in \mathrm{C} \quad::=(t, e) \\
& \text { Stacks } \pi \in \Pi \quad::=\star|\langle c, \pi\rangle| \operatorname{kp}_{i}(\pi)|\operatorname{ksucc}(\pi)| \operatorname{krec}(t, u, c, \pi)
\end{aligned}
$$


We let $e(x):=c$ if $(x, c)$ is the first occurrence of the form $\left(x, c^{\prime}\right)$ in $e$, and define $e(\alpha)$ similarly. Let $\operatorname{dom}(e)$ be the domain of the partial map $e(-)$.

The evaluation rules are the following:

$$
\begin{array}{rlr}
(x, e, \pi) & \succ\left(t, e^{\prime}, \pi\right) & \text { if } e(x)=\left(t, e^{\prime}\right) \\
(t u, e, \pi) & \succ(t, e,\langle(u, e), \pi\rangle) & \\
(\lambda x . t, e,\langle c, \pi\rangle) & \succ(t,(x, c):: e, \pi) & \\
(\mu \alpha \cdot[\beta] t, e, \pi) & \succ\left(t,(\alpha, \pi):: e, \pi^{\prime}\right) & \text { if }((\alpha, \pi):: e)(\beta)=\pi^{\prime} \\
\left(\mathrm{p}_{i}(t), e, \pi\right) & \succ\left(t, e, \mathrm{kp}_{i}(\pi)\right) & i=1,2 \\
\left(\left\langle t_{1}, t_{2}\right\rangle, e, \mathrm{kp}_{i}(\pi)\right) & \succ\left(t_{i}, e, \pi\right) & i=1,2 \\
\left(\operatorname{succ}, e,\left\langle\left(t, e^{\prime}\right), \pi\right\rangle\right) & \succ\left(t, e^{\prime}, \operatorname{ksucc}(\pi)\right) & \\
(\bar{n}, e, \operatorname{ksucc}(\pi)) & \succ(n+1, e, \pi) & \\
\left(\operatorname{rec}(t, u), e,\left\langle\left(v, e^{\prime}\right), \pi\right\rangle\right) & \succ\left(v, e^{\prime}, \operatorname{krec}(t, u, e, \pi)\right) & \\
\left(\overline{0}, e, \operatorname{krec}\left(t, u, e^{\prime}, \pi\right)\right) & \succ\left(t, e^{\prime}, \pi\right) & \\
\left(\overline{n+1}, e, \operatorname{krec}\left(t, u, e^{\prime}, \pi\right)\right) & \succ\left(u, e^{\prime},\left\langle(\bar{n}, e),\left\langle\left(\operatorname{rec}(t, u) \bar{n}, e^{\prime}\right), \pi\right\rangle\right\rangle\right)
\end{array}
$$

The correctness of the machine (i.e. reduction preserves semantics) can be proved as usual ${ }^{3}$ (see e.g. [18]). For extraction, we actually only need the property stated in Prop. 7.3, to be discussed in presence of Bar-Recursion.

\section{Classical Realizability}

In this section, we present our notion of realizability. It is highly inspired from Krivine's Realizability [10], but adapted to the simply-typed model $\mathrm{R}^{\mathrm{Fam}(\mathcal{G}) \text {. }}$

The main idea, adapting Krivine's ideas to the typed continuation category $\mathrm{R}^{\mathrm{Fam}(\mathcal{G})}$, would be to fix a Pole $\Perp \subseteq\{[\bar{n}] \mid n \in \mathbb{N}\}$, and then associate to each formula $A$ a type $A^{*}$ and a set $\mathcal{A} \subseteq \llbracket A^{*} \rrbracket$ defined by induction on $A$. Realizers would then be terms in $\mathcal{A}^{\Perp} \subseteq\left[A^{*}\right]$, the Orthogonal of $\mathcal{A}$.

We choose to have $\Perp \subseteq[\iota]$ to get extraction (see Prop. 7.4). This causes difficulties since $[\iota]=R^{\llbracket \iota \rrbracket}$ is not a base type in $R^{\mathrm{Fam}(\mathcal{G})}$. Roughly speaking, our choice for $\Perp$ leads to $\perp^{*}:=\iota$, but there are not enough contexts in $\llbracket \iota \rrbracket=\left\{\perp_{\llbracket \iota \rrbracket}\right\}$, since applying $\perp_{\llbracket \iota \rrbracket}$ to a numeral $[\bar{n}]$ gives the empty strategy on $\mathrm{R}$. A solution is to add some space in the interpretations, and have $\mathcal{A} \subseteq \llbracket \iota \rrbracket \rightarrow \llbracket A^{*} \rrbracket$ and $\mathcal{A}^{\Perp} \subseteq \llbracket \iota \rrbracket \rightarrow\left[A^{*}\right]$ for a formula $A$. For instance, we can then have $\boldsymbol{\lambda} k . k$ as a basic context "at type" $\llbracket \iota$ (actually $\llbracket \iota \rrbracket \rightarrow \llbracket \iota \rrbracket)$.

The definition of realizability involves two additional translations, that we present now. First, to each formula $A$, we associate the simple type $A^{*}$ :

$$
\begin{array}{cccc}
\left(a^{\tau} \neq_{\tau} b^{\tau}\right)^{*}:=\iota \quad \perp^{*}:=\iota & \left(\forall x^{\tau} A\right)^{*}:=\tau \rightarrow A^{*} \\
(A \Rightarrow B)^{*}:=A^{*} \rightarrow B^{*} & & (A \wedge B)^{*}:=A^{*} \times B^{*}
\end{array}
$$

\footnotetext{
${ }^{3}$ Since the model $\mathrm{R}^{\mathrm{Fam}(\mathcal{G})}$ is typed, this would involve typing rules for environments and stacks.
} 
Moreover, we map each individual term $a \in \mathcal{I}$ to a $\lambda \mu$-term $a^{\dagger} \in \Lambda_{T}$ :

$$
\begin{aligned}
& x^{\tau^{\dagger}}:=x \quad(a b)^{\dagger}:=a^{\dagger} b^{\dagger} \quad \mathrm{s}^{\dagger}:=\lambda x y z \cdot x z(y z) \\
& \mathrm{k}^{\dagger}:=\lambda x y \cdot x \quad 0^{\dagger}:=\overline{0} \quad \mathrm{~S}^{\dagger}:=\text { succ } \\
& \operatorname{Rec}^{\dagger}:=\lambda x y \cdot \operatorname{rec}(x, y) \quad \text { Pair }^{\dagger}:=\lambda x y \cdot\langle x, y\rangle \quad \mathrm{P}_{i}^{\dagger}:=\lambda x \cdot \mathrm{p}_{i}(x)
\end{aligned}
$$

The Realizability Construction. To a formula $A$, we will associate two sets $\|A\| \subseteq \llbracket \iota \rrbracket \rightarrow \llbracket A^{*} \rrbracket$ and $|A| \subseteq \llbracket \iota \rrbracket \rightarrow\left[A^{*}\right]$. These sets will only be defined for closed formulas. It is convenient (and necessary to deal with CAC in Sect. 7) to allow parameters in $\mathrm{R}^{\mathrm{Fam}(\mathcal{G})}$. In order to realize the induction axiom, we must restrict to the total elements of $\mathrm{R}^{\mathrm{Fam}(\mathcal{G})}$. For a simple type $\tau$, the set $\tau^{\mathrm{t}} \subseteq[\tau]$ of its total elements is defined by induction on $\tau$. Let $\iota^{\mathrm{t}}:=\{[\bar{n}] \mid n \in \mathbb{N}\}$, and using curryfied notation:

$$
\begin{aligned}
(\sigma \rightarrow \tau)^{\mathrm{t}} & :=\left\{a \mid \forall b \in \sigma^{\mathrm{t}}, a b \in \tau^{\mathrm{t}}\right\} \\
(\sigma \times \tau)^{\mathrm{t}} & :=\left\{a \mid \mathbf{p}_{1}(a) \in \sigma^{\mathrm{t}} \& \mathbf{p}_{2}(a) \in \tau^{\mathrm{t}}\right\}
\end{aligned}
$$

Lemma 6.1. For all $a \in \mathcal{I}_{0}^{\tau},\left[a^{\dagger}\right] \in \tau^{\mathrm{t}}$.

We now only consider closed formulas with parameters of the appropriate type in $\tau^{\mathrm{t}}(\tau \in \mathcal{T})$. Let $\Perp \subseteq \iota^{\mathrm{t}}$.

First, given $\mathcal{A} \subseteq \llbracket \iota \rrbracket \rightarrow \llbracket A^{*} \rrbracket$, we define $\mathcal{A}^{\Perp} \subseteq \llbracket \iota \rrbracket \rightarrow\left[A^{*}\right]$ as

$$
\mathcal{A}^{\Perp} \quad:=\quad\left\{a \in \llbracket \iota \rrbracket \rightarrow\left[A^{*}\right] \mid \forall b \in \mathcal{A}, \quad \boldsymbol{\lambda} k \cdot a k(b k) \in \Perp\right\}
$$

If moreover $\mathcal{B} \subseteq \llbracket \iota \rrbracket \rightarrow \llbracket \mathcal{B}^{*} \rrbracket$, we let

$$
\mathcal{A}^{\Perp} \cdot \mathcal{B} \quad:=\quad\left\{\boldsymbol{\lambda} k \cdot\langle a k, b k\rangle \in \llbracket \iota \rrbracket \rightarrow\left[A^{*}\right] \times \llbracket B^{*} \rrbracket \mid a \in \mathcal{A}^{\Perp} \& b \in \mathcal{B}\right\}
$$

We now define the sets $|A| \subseteq \llbracket \iota \rrbracket \rightarrow\left[A^{*}\right]$ and $\|A\| \subseteq \llbracket \iota \rrbracket \rightarrow \llbracket A^{*} \rrbracket$ for a formula $A$. We let $|A| \subseteq \llbracket \iota \rrbracket \rightarrow\left[A^{*}\right]$ be $\|A\|^{\Perp}$, and define $\|A\| \subseteq \llbracket \iota \rrbracket \rightarrow \llbracket A^{*} \rrbracket$ by induction on $A$ as follows:

$$
\begin{aligned}
\|\perp\| & :=\{\boldsymbol{\lambda} k . k\} \quad\|\quad\| A \Rightarrow B\|\quad:=|A| \cdot\| B \| \\
\left\|a \neq_{\tau} b\right\| & := \begin{cases}\emptyset & \text { if }\left[a^{\dagger}\right] \neq\left[b^{\dagger}\right] \\
\{\boldsymbol{\lambda} k . k\} & \text { otherwise }\end{cases} \\
\|A \wedge B\| & :=\left\{\boldsymbol{\lambda} k . \mathbf{i n}_{1}(a k) \mid a \in\|A\|\right\} \cup\left\{\boldsymbol{\lambda} k . \mathbf{i n}_{2}(b k) \mid b \in\|B\|\right\} \\
\left\|\forall x^{\tau} A\right\| & :=\bigcup_{a \in \tau^{\mathrm{\tau}}}\{\boldsymbol{\lambda} k .\langle a, b k\rangle \mid b \in\|A[a / x]\|\}
\end{aligned}
$$

Realization of Equality and Arithmetic Axioms. We now discuss the realization of the axioms of $\mathrm{PA}^{\omega}$.

First, it is easy to see that all equational axioms (including reflexivity) are realized by the identity:

Lemma 6.2. We have $\boldsymbol{\lambda} k .[\lambda x . x] \in\left|a=_{\tau} a\right|$. Moreover,

$$
\begin{array}{ccc}
\lambda k \cdot[\lambda x \cdot x] \in\left|\mathrm{k} a b={ }_{\tau} a\right| & \lambda k \cdot[\lambda x \cdot x] \in\left|\mathrm{s} a b c={ }_{\tau} a c(b c)\right| \\
\lambda k \cdot[\lambda x \cdot x] \in\left|\operatorname{Rec} a b 0={ }_{\tau} a\right| & \lambda k \cdot[\lambda x \cdot x] \in \mid \operatorname{Rec} a b(\mathrm{~S} c) & ={ }_{\tau} b c(\operatorname{Rec} a b c) \mid
\end{array}
$$

where in each case, individuals $a, b, c$ are in the appropriate $\tau^{\mathrm{t}}, \sigma^{\mathrm{t}}, \rho^{\mathrm{t}}$. 
The realization of our version of Leibniz's scheme is obtained by applying realizers of the first premise to realizers of the second premise.

Lemma 6.3. $\lambda k$. $[\lambda x . \lambda y . y x] \in\left|A\left[a^{\tau} / z^{\tau}\right] \Rightarrow \neg A\left[b^{\tau} / z^{\tau}\right] \Rightarrow a^{\tau} \neq_{\tau} b^{\tau}\right|$.

For the Arithmetic axioms, it is easy to see that $(\mathrm{S} a \neq \iota)$ is realized by any natural number. As expected, the recursor rec $\left({ }_{-},-\right)$realizes induction.

Lemma 6.4. (i) For all $n \in \mathbb{N}$ and all $a \in \iota^{\mathrm{t}}$, we have $\boldsymbol{\lambda} k \cdot[\bar{n}] \in \mid \mathrm{S} a \neq \iota$. $0 \mid$.

(ii) $\quad \lambda k \cdot[\lambda x \cdot \lambda y \cdot \operatorname{rec}(x, y)] \in\left|A[0 / x] \Rightarrow \forall x^{\iota}(A \Rightarrow A[\mathrm{~S} x / x]) \Rightarrow \forall x^{\iota} A\right|$.

Adequacy for Classical Proofs. Adequacy of the realizability interpretation is proved as usual (see App. A).

Theorem 6.5. Let $\Gamma, A, \Delta$ with $\Gamma=A_{1}, \ldots, A_{n}, \Delta=B_{1}, \ldots, B_{m}$, and such that $F V(\Gamma, A, \Delta) \subseteq\left\{x_{1}^{\tau_{1}}, \ldots, x_{k}^{\tau_{k}}\right\}$.

From a proof of $\Gamma \vdash A \mid \Delta$ in $\mathrm{PA}^{\omega}$ one can build a term

$$
x_{1}: \tau_{1}, \ldots, x_{k}: \tau_{k}, y_{1}: A_{1}{ }^{*}, \ldots, y_{n}: A_{n}{ }^{*} \vdash t: A^{*} \mid \alpha_{1}: B_{1}{ }^{*}, \ldots, \alpha_{m}: B_{m}{ }^{*}
$$

such that for all $c_{1} \in \tau_{1}{ }^{\mathrm{t}}, \ldots, c_{k} \in \tau_{k}{ }^{\mathrm{t}}$, all $a_{1} \in\left|A_{1}[\boldsymbol{c} / \boldsymbol{x}]\right|, \ldots, a_{n} \in\left|A_{n}[\boldsymbol{c} / \boldsymbol{x}]\right|$, and all $b_{1} \in\left\|B_{1}[\boldsymbol{c} / \boldsymbol{x}]\right\|, \ldots, b_{m} \in\left\|B_{m}[\boldsymbol{c} / \boldsymbol{x}]\right\|$, we have

$$
\boldsymbol{\lambda} k \cdot[t][\boldsymbol{c} / \boldsymbol{x}]\left[a_{1} k / y_{1}, \ldots, a_{n} k / y_{n}, b_{1} k / \alpha_{1}, \ldots, b_{m} k / \alpha_{m}\right] \in|A[\boldsymbol{c} / \boldsymbol{x}]|
$$

In particular, from a proof of $\vdash A \mid$ in $\mathrm{PA}^{\omega}$ with $A$ closed, one can build a term $\vdash t: A^{*} \mid$ such that $\lambda k .[t] \in|A|$.

Extraction. Extraction of witnessing programs from realizable (and hence from provable) $\Pi_{2}^{0}$ statements is performed as usual. We come back on this point in Sect. 7 (Prop. 7.4) in presence of CAC and Bar-Recursion.

\section{Realization of Classical Choice}

In this section we discuss the realization of the classical axiom of choice $\mathrm{CAC}^{\ell,-}$. Our realizer is based on Berger \& Oliva's variant of Spector's Bar-Recursion [4].

Extension of the $\boldsymbol{\lambda} \boldsymbol{\mu}$-Calculus with Bar-Recursion. We extend the set $\Lambda_{T}$ with constants for bar-recursion: $t, u \in \Lambda_{\Psi}::=\ldots \mid \Psi_{\tau}(t, u)\left\langle s_{0}, \ldots, s_{n}\right\rangle$, where $n \in \mathbb{N}$ and $\tau \in \mathcal{T}$.

These constants are typed as follows: $\Gamma \vdash \Psi_{\tau}(t, u)\left\langle s_{0}, \ldots, s_{n}\right\rangle: \iota \mid \Delta$ whenever $\Gamma \vdash t: \iota \rightarrow(\tau \rightarrow \iota) \rightarrow \iota|\Delta, \Gamma \vdash u:(\iota \rightarrow \tau) \rightarrow \iota| \Delta$ and $\Gamma \vdash s_{i}: \tau \mid \Delta$ for all $0 \leq i \leq n$.

The operational semantics uses some auxiliary terms. We define by induction on $\tau$ the terms $\vdash \mathrm{ex}_{\tau}: \iota \rightarrow \tau \mid$. Let $\mathrm{ex}_{\iota}:=\lambda x . x, \mathrm{ex}_{\tau \rightarrow \sigma}:=\lambda x \cdot \lambda_{-} \cdot \mathrm{ex}_{\sigma} x$ and $\mathrm{ex}_{\tau \times \sigma}:=\lambda x \cdot\left\langle\operatorname{ex}_{\tau} x, \mathrm{ex}_{\sigma} x\right\rangle$. 
Moreover, given $n \in \mathbb{N}, s_{0}, \ldots, s_{n}, t \in \Lambda_{\Psi}$, we let $\left\langle s_{0}, \ldots, s_{n}\right\rangle @ t$ be a term (written using rec) such that for all $e, e^{\prime} \in \mathrm{E}, \pi \in \Pi$ and $m \in \mathbb{N}$,

$$
\left(\left\langle s_{0}, \ldots, s_{n}\right\rangle @ t, e,\left\langle\left(\bar{m}, e^{\prime}\right), \pi\right\rangle\right) \succ \begin{cases}\left(s_{m}, e, \pi\right) & \text { if } m \leq n \\ \left(t, e,\left\langle\left(\overline{m-(n+1)}, e^{\prime}\right), \pi\right\rangle\right) & \text { otherwise }\end{cases}
$$

The operational semantics of $\Psi_{\tau}(t, u)\left\langle s_{1}, \ldots, s_{n}\right\rangle$ is given by:

$$
\begin{aligned}
&\left(\Psi_{\tau}(t, u)\left\langle s_{0}, \ldots, s_{n}\right\rangle, e, \pi\right) \succ \\
&\left(u, e,\left\langle\left(\left\langle s_{0}, \ldots, s_{n}\right\rangle @ \lambda_{-} . \operatorname{ex}_{\tau}\left(t \overline{n+1} \lambda x \cdot \Psi_{\tau}(t, u)\left\langle s_{0}, \ldots, s_{n}, x\right\rangle\right), e\right), \pi\right\rangle\right)
\end{aligned}
$$

The Bar-Recursor in $\mathrm{R}^{\mathrm{Fam}(\mathcal{G})}$. We now define the strategies interpreting $\Psi_{\tau}$ in $\mathrm{R}^{\mathrm{Fam}(\mathcal{G})}$. Fix $\tau \in \mathcal{T}$. First, given $a_{0}, \ldots, a_{n} \in[\tau]$, and $b \in[\iota \rightarrow \tau]$, let

$$
\left\langle a_{0}, \ldots, a_{n}\right\rangle @ b:=\left[\left\langle x_{0}, \ldots, x_{n}\right\rangle @ y\right]\left[a_{0} / x_{0}, \ldots, a_{n} / x_{n}, b / y\right]
$$

For each $m \in \mathbb{N}$, we will define by induction on $m$ a family of strategies $\left(\widetilde{\Psi}_{n}^{m}\right)_{n \in \mathbb{N}}$. Each $\widetilde{\Psi}_{n}^{m}$ will be in $[\tau]^{n} \rightarrow \widetilde{\tau_{\Psi}}$, where $[\tau]^{0}:=\{\mathcal{U}\},[\tau]^{n+1}:=[\tau] \times[\tau]^{n}$ and

$$
\widetilde{\tau_{\Psi}}:=[\iota \rightarrow(\tau \rightarrow \iota) \rightarrow \iota] \rightarrow[(\iota \rightarrow \tau) \rightarrow \iota] \rightarrow[\iota]
$$

We let $\widetilde{\Psi}_{n}^{0}:=\boldsymbol{\lambda}\left\langle x_{1}, \ldots, x_{n}\right\rangle \cdot \perp_{\widetilde{\tau_{\Psi}}}$ and

$$
\begin{aligned}
\widetilde{\Psi}_{n}^{m+1}:=\quad \boldsymbol{\lambda}\left\langle x_{1}, \ldots, x_{n}\right\rangle \cdot \boldsymbol{\lambda} b \cdot \boldsymbol{\lambda} c . c^{\bullet}\left(\left\langle x_{1}, \ldots, x_{n}\right\rangle @\right. \\
\left.\boldsymbol{\lambda}_{-.}\left[\mathrm{ex}_{\tau}\right]^{\bullet}\left(b^{\bullet}[\overline{n+1}]\left(\boldsymbol{\lambda} x . \widetilde{\Psi}_{n}^{m}\left\langle x_{1}, \ldots, x_{n}, x\right\rangle b c\right)^{\circ}\right)\right)
\end{aligned}
$$

Given $a_{0}, \ldots, a_{n} \in[\tau]$, we now define a strategy $\widetilde{\Psi}_{\left\langle a_{0}, \ldots, a_{n}\right\rangle}^{\tau}$ using the CPO structure on $\mathcal{G}$ (and hence on $\operatorname{Fam}(\mathcal{G})$ ). Note that the family $\left(\widetilde{\Psi}_{n+1}^{m}\left\langle a_{0}, \ldots, a_{n}\right\rangle\right)_{m \in \mathbb{N}}$ is directed. We let $\widetilde{\Psi}_{\left\langle a_{0}, \ldots, a_{n}\right\rangle}^{\tau}:=\bigvee_{m \in \mathbb{N}} \widetilde{\Psi}_{n+1}^{m}\left\langle a_{0}, \ldots, a_{n}\right\rangle$ and $\left[\Psi_{\tau}(t, u)\left\langle s_{0}, \ldots, s_{n}\right\rangle\right]:=\widetilde{\Psi}_{\left\langle\left[s_{0}\right], \ldots,\left[s_{n}\right]\right\rangle}^{\tau}[t][u]$.

Realization of CAC ${ }^{\iota,-}$. We discuss here the realization of CAC $^{\iota, \tau}$ using (the interpretation in $\Lambda_{\Psi}$ ) of the term $t_{\text {CAC }}$ build in Sect. 3, where we take suitable instances of $\Psi_{\tau}\left({ }_{-},-\right)\langle\ldots\rangle$ for Bar-Recursion. We let

$t_{\text {CAC }}^{\tau, A}:=\lambda z \cdot \lambda c \cdot \Psi_{\tau \times A^{*}}\left(t_{\neg \neg \exists} z, \lambda a \cdot c\left(\lambda x \cdot \mathrm{p}_{1}(a x)\right)\left(\lambda x \cdot \mathrm{p}_{2}(a x)\right)\right)\langle\rangle$

Proposition 7.1. $\lambda k \cdot\left[t_{\text {CAC }}^{\tau, A}\right] \in$ $\left|\forall x^{\iota}\left(\forall y^{\tau}(A \Rightarrow \perp) \Rightarrow \perp\right) \stackrel{\Rightarrow}{\Rightarrow} f^{\iota \rightarrow \tau}\left(\forall x^{\iota} A[f x / y] \Rightarrow \perp\right) \Rightarrow \perp\right|$.

The proof of Prop. 7.1 is deferred to App. C. Contrary to e.g. [3, 4], we do not rely on the decomposition of CAC as IAC + DNS discussed in Sect. 3. Rather, we show directly that Bar-Recursion realizes a form of choice. 
The main point is to decompose the notion of realizability proposed in Sect. 6 w.r.t. the relativization of quantifiers. We first extend the formulas:

$$
A, B \quad:=\ldots\left|\tilde{\forall} x^{\tau} A \quad\right| \quad\left(\mathrm{r}_{\tau}\left(a^{\tau}\right) \times A\right) \Rightarrow B
$$

Hence, in extended formulas, the construction $\left(r_{\tau}(a) \times A\right)$ is only allowed to appear to the left of an implication. Realizability is extended as follows:

$$
\begin{aligned}
\left\|\tilde{\forall} x^{\tau} A\right\| & :=\bigcup_{a \in \tau^{\mathrm{t}}}\|A[a / x]\| \\
\left\|\left(\mathrm{r}_{\tau}(c) \times A\right) \Rightarrow B\right\| & :=\left\{\boldsymbol{\lambda} k .\left\langle\boldsymbol{\lambda} k^{\prime} \text {.case } k^{\prime}\{c, a k\}, b k\right\rangle|a \in| A \mid \& b \in\|B\|\right\}
\end{aligned}
$$

Extended formulas and their realizability interpretation rely on ideas introduced in Krivine's Realizability [10] (see also [12]). We also extend the mapping $\left({ }_{-}\right)^{*}$ :

$$
\left(\tilde{\forall} x^{\tau} A\right)^{*}:=A^{*} \quad\left(\left(\mathrm{r}_{\tau}(a) \times A\right) \Rightarrow B\right)^{*} \quad:=\tau \times A^{*} \rightarrow B^{*}
$$

The following is the key for Prop. 7.1. It is shown as usual, see e.g. [3, 4].

Lemma 7.2. Let $B$ such that $(B \Rightarrow \perp)$ is an extended formula.

Assume $b \in\left|\forall x^{\iota}\left(\tilde{\forall} y^{\tau}(B \Rightarrow \perp) \Rightarrow \perp\right)\right|$ and $c \in\left|\tilde{\forall} f^{\iota \rightarrow \tau}\left(\forall x^{\iota} B[f x / y] \Rightarrow \perp\right)\right|$.

Then $\boldsymbol{\lambda} k . \widetilde{\Psi}_{\langle\rangle}^{B^{*}}(b k)(c k) \in|\perp|$.

Computational Adequacy and Extraction. For extraction, we rely on the following property relating the evaluation of $\lambda \mu$-terms with their interpretation in $\mathrm{R}^{\mathrm{Fam}(\mathcal{G})}$. The proof is deferred to App. B.

Proposition 7.3. (i) If $\vdash t: \iota \mid$ in $\Lambda_{\Psi}$, then for all $n \in \mathbb{N}$ we have $(t, \varepsilon, \star) \succ$ $(\bar{n}, e, \star)$ if $[t]=[\bar{n}]$.

(ii) Let $\vdash t: \iota \rightarrow \iota$ in $\Lambda_{\Psi}$. For all $n, m \in \mathbb{N}$, if $\boldsymbol{\lambda} k .[t]\langle[\bar{n}], k\rangle=[\bar{m}]$ then $(t \bar{n}, \varepsilon, \star) \succ(\bar{m}, e, \star)$.

Extraction of witnessing programs from realizable (and hence from provable) $\Pi_{2}^{0}$ statements is performed as usual:

Proposition 7.4. From a proof of $\mathrm{PA}^{\omega}+\mathrm{CAC}^{\iota,-} \vdash \forall x^{\iota} \exists y^{\iota}\left(a={ }_{\iota} 0\right)$ (where $F V(a) \subseteq\{x, y\})$, we can extract a term $\vdash t: \iota \rightarrow \iota \mid$ such that for all $n \in \mathbb{N}$, there is $m \in \mathbb{N}$ such that $(t \bar{n}, \varepsilon, \star) \succ(\bar{m}, e, \star)$ and $\left[a^{\dagger}\right][[\bar{n}] / x,[\bar{m}] / y]=[\overline{0}]$.

Proof (sketch). By adequacy, we get $u$ s.t. $\lambda k$. $[u] \in\left|\forall x^{\iota} \neg \forall y^{\iota}(a \neq \iota)\right|$. Let $n \in \mathbb{N}$ and fix $\Perp:=\left\{[\bar{m}] \mid\left[a^{\dagger}\right][[\bar{n}] / x,[\bar{m}] / y]=[\overline{0}]\right\}$. We thus have $\boldsymbol{\lambda} k .[u \bar{n}(\lambda x . x)] \in|\perp|$. This implies $[u \bar{n}(\lambda x . x)]=[\bar{m}]$ with $[\bar{m}] \in \Perp$. We conclude by Prop. 7.3.(ii).

\section{Conclusion}

We presented a notion of classical realizability for PA ${ }^{\omega}+$ CAC based on HylandOng innocent unbracketed games for a simply-typed extension of Parigot's $\lambda \mu$ calculus. It is not clear whether in our approach decomposes CAC as IAC+DNS, because of the interaction of the CPS translation with Friedman's trick.

Further works will concern this question, a comparison with [17], where BarRecursion is used in an untyped Classical Realizablity model, as well as trying to extend the result to non-innocent games (along the lines of [5]), known to rise problems with Bar-Recursion [3]. 


\section{References}

[1] S. Abramsky and G. McCusker. Call-by-Value Games. In Proceedings of CSL'97, LNCS, pages 1-17. Springer, 1997.

[2] R. M. Amadio and P.-L. Curien. Domains and Lambda-Calculi. Cambridge Tracts in Theoretical Computer Science. Cambridge University Press, 1998.

[3] S. Berardi, M. Bezem, and T. Coquand. On the Computational Content of the Axiom of Choice. Journal of Symbolic Logic, 63(2):600-622, 1998.

[4] U. Berger and P. Oliva. Modified bar recursion and classical dependent choice. Lecture Notes in Logic, 20:89-107, 2005.

[5] V. Blot. Realizability for Peano Arithmetic with Winning Conditions in HO Games. To appear in Proceedings of TLCA'13, 2013.

[6] T. Griffin. A Formulae-as-Types Notion of Control. In POPL'90, pages 47-58. ACM Press, 1990.

[7] R. Harmer. Games and Full Abstraction for Nondeterministic Languages. PhD thesis, Imperial College, London, 1999.

[8] J. M. E. Hyland and C.-H. Ong. On Full Abstraction for PCF: I, II, and III. Information and Computation, 163(2):285-408, 2000.

[9] U. Kohlenbach. Applied Proof Theory: Proof Interpretations and their Use in Mathematics. Springer Monographs in Mathematics. Springer, 2008.

[10] J.-L. Krivine. Realizability in classical logic. In Interactive models of computation and program behaviour, volume 27 of Panoramas et synthèses, pages 197-229. Société Mathématique de France, 2009.

[11] J. Laird. A Semantic analysis of control. PhD thesis, University of Edimbourgh, 1998.

[12] A. Miquel. Existential witness extraction in classical realizability and via a negative translation. Logical Methods in Computer Science, 7(2), 2011.

[13] P. Oliva and T. Streicher. On Krivine's Realizability Interpretation of Classical Second-Order Arithmetic. Fundam. Inform., 84(2):207-220, 2008.

[14] M. Parigot. Lambda-My-Calculus: An Algorithmic Interpretation of Classical Natural Deduction. In $L P A R$, volume 624 of $L N C S$, pages 190-201. Springer, 1992.

[15] P. Selinger. Control Categories ans Duality: on the Categorical Semantics of the Lambda-Mu Calculus. Mathematical Structures in Computer Science, 11:207-260, 2001.

[16] S. G. Simpson. Subsystems of Second Order Arithmetic. Perspectives in Logic. Cambridge University Press, 2nd edition, 2010.

[17] T. Streicher. A Classical Realizability Model araising from a Stable Model of Untyped Lambda-Calculus. Unpublished Notes, 2013.

[18] T. Streicher and B. Reus. Classical Logic, Continuation Semantics and Abstract Machines. J. Funct. Program., 8(6):543-572, 1998.

[19] A. S. Troelstra. Metamathematical Investigation of Intuitionistic Arithmetic and Analysis, volume 344 of LNM. Springer Verlag, 1973. 


\section{A Adequacy of the Realizability Interpretation}

In this appendix, we give a proof of adequacy of our realizability interpretation (Thm. 6.5).

Lemma A.1. If $c \in \llbracket \iota \rrbracket \rightarrow\left[\tau \rightarrow A^{*}\right]$, then:

$$
c \in\left|\forall x^{\tau} A\right| \Leftrightarrow \forall e \in \tau^{\mathrm{t}}, \boldsymbol{\lambda} k \cdot[x y][c k / x, e / y] \in|A[e / x]|
$$

Proof. One one hand:

$$
\begin{aligned}
c \in\left|\forall x^{\tau} A\right| & \Leftrightarrow \quad \forall d \in\left\|\forall x^{\tau} A\right\|, \boldsymbol{\lambda} k \cdot c k(d k) \in \Perp \\
& \Leftrightarrow \quad \forall e \in \tau^{\mathrm{t}}, \forall b \in\|A[e / x]\|, \boldsymbol{\lambda} k \cdot c k\left(\left(\boldsymbol{\lambda} k^{\prime} .\left\langle e, b k^{\prime}\right\rangle\right) k\right) \in \Perp \\
& \Leftrightarrow \quad \forall e \in \tau^{\mathrm{t}}, \forall b \in\|A[e / x]\|, \boldsymbol{\lambda} k . c k\langle e, b k\rangle \in \Perp
\end{aligned}
$$

and on the other hand if $e \in \tau^{\mathrm{t}}$ and $b \in\|A[e / x]\|$ then:

$$
\begin{aligned}
\boldsymbol{\lambda} k \cdot\left(\boldsymbol{\lambda} k^{\prime} \cdot[x y]\left[c k^{\prime} / x, e / y\right]\right) k(b k) & =\boldsymbol{\lambda} k \cdot([x y][c k / x, e / y])(b k) \\
& =\boldsymbol{\lambda} k \cdot\left(\left(\boldsymbol{\lambda} k^{\prime} \cdot x\left\langle y, k^{\prime}\right\rangle\right)[c k / x, e / y]\right)(b k) \\
& =\boldsymbol{\lambda} k \cdot\left(\boldsymbol{\lambda} k^{\prime} \cdot c k\left\langle e, k^{\prime}\right\rangle\right)(b k) \\
& =\boldsymbol{\lambda} k \cdot c k\langle e, b k\rangle
\end{aligned}
$$

therefore:

$$
c \in|A \Rightarrow B| \quad \Leftrightarrow \quad \forall e \in \tau^{\mathrm{t}}, \forall b \in\|A[e / x]\|, \boldsymbol{\lambda} k \cdot\left(\boldsymbol{\lambda} k^{\prime} \cdot[x y]\left[c k^{\prime} / x, e / y\right]\right) k(b k) \in \Perp
$$

that is:

$$
c \in|A \Rightarrow B| \quad \Leftrightarrow \quad \forall e \in \tau^{\mathrm{t}}, \boldsymbol{\lambda} k^{\prime} \cdot[x y]\left[c k^{\prime} / x, e / y\right] \in|A[e / x]|
$$

Lemma A.2. If $A$ is such that $F V(A)=\left\{x^{\tau}\right\}$ and if for all $e \in \tau^{\mathrm{t}}, b \in|A[e / x]|$, then $\boldsymbol{\lambda} k \cdot[\lambda x . y][b k / y] \in\left|\forall x^{\tau} A\right|$.

Proof. From lemma A.1 we have:

$\boldsymbol{\lambda} k \cdot[\lambda x . y][b k / y] \in\left|\forall x^{\tau} A\right| \Leftrightarrow \forall e \in \tau^{\mathrm{t}}, \boldsymbol{\lambda} k \cdot\left[x y^{\prime}\right]\left[\left(\boldsymbol{\lambda} k^{\prime} .[\lambda x . y]\left[b k^{\prime} / y\right]\right) k / x, e / y^{\prime}\right] \in|A[e / x]|$ but since:

$$
\begin{aligned}
\boldsymbol{\lambda} k \cdot\left[x y^{\prime}\right]\left[\left(\boldsymbol{\lambda} k^{\prime} \cdot[\lambda x \cdot y]\left[b k^{\prime} / y\right]\right) k / x, e / y^{\prime}\right] & =\boldsymbol{\lambda} k \cdot\left[x y^{\prime}\right]\left[[\lambda x \cdot y][b k / y] / x, e / y^{\prime}\right] \\
& =\boldsymbol{\lambda} k \cdot\left[(\lambda x \cdot y) y^{\prime}\right]\left[b k / y, e / y^{\prime}\right] \\
& =\boldsymbol{\lambda} k \cdot[y]\left[b k / y, e / y^{\prime}\right] \\
& =\boldsymbol{\lambda} k \cdot y\left[b k / y, e / y^{\prime}\right] \\
& =\boldsymbol{\lambda} k \cdot b k \\
& =b
\end{aligned}
$$

We have:

$$
\lambda k .[\lambda x . y][b k / y] \in\left|\forall x^{\tau} A\right| \Leftrightarrow \forall e \in \tau^{\mathrm{t}}, b \in|A[e / x]|
$$

from which we conclude since $b \in|A[e / x]|$. 
Lemma A.3. If $c \in \llbracket \iota \rrbracket \rightarrow\left[A^{*} \rightarrow B^{*}\right]$, then:

$$
c \in|A \Rightarrow B| \Leftrightarrow \forall a \in|A|, \lambda k \cdot[x y][c k / x, a k / y] \in|B|
$$

Proof. One one hand:

$$
\begin{aligned}
c \in|A \Rightarrow B| & \Leftrightarrow \quad \forall d \in\|A \Rightarrow B\|, \boldsymbol{\lambda} k . c k(d k) \in \Perp \\
& \Leftrightarrow \quad \forall a \in|A|, \forall b \in\|B\|, \boldsymbol{\lambda} k . c k\left(\left(\boldsymbol{\lambda} k^{\prime} .\left\langle a k^{\prime}, b k^{\prime}\right\rangle\right) k\right) \in \Perp \\
& \Leftrightarrow \quad \forall a \in|A|, \forall b \in\|B\|, \boldsymbol{\lambda} k . c k\langle a k, b k\rangle \in \Perp
\end{aligned}
$$

and on the other hand if $a \in|A|$ and $b \in\|B\|$ then:

$$
\begin{aligned}
\boldsymbol{\lambda} k \cdot\left(\boldsymbol{\lambda} k^{\prime} \cdot[x y]\left[c k^{\prime} / x, a k^{\prime} / y\right]\right) k(b k) & =\boldsymbol{\lambda} k \cdot([x y][c k / x, a k / y])(b k) \\
& =\boldsymbol{\lambda} k \cdot\left(\left(\boldsymbol{\lambda} k^{\prime} \cdot x\left\langle y, k^{\prime}\right\rangle\right)[c k / x, a k / y]\right)(b k) \\
& =\boldsymbol{\lambda} k \cdot\left(\boldsymbol{\lambda} k^{\prime} \cdot c k\left\langle a k, k^{\prime}\right\rangle\right)(b k) \\
& =\boldsymbol{\lambda} k \cdot c k\langle a k, b k\rangle
\end{aligned}
$$

therefore:

$$
c \in|A \Rightarrow B| \quad \Leftrightarrow \quad \forall a \in|A|, \forall b \in\|B\|, \boldsymbol{\lambda} k .\left(\boldsymbol{\lambda} k^{\prime} \cdot[x y]\left[c k^{\prime} / x, a k^{\prime} / y\right]\right) k(b k) \in \Perp
$$

that is:

$$
c \in|A \Rightarrow B| \quad \Leftrightarrow \quad \forall a \in|A|, \boldsymbol{\lambda} k^{\prime} .[x y]\left[c k^{\prime} / x, a k^{\prime} / y\right] \in|B|
$$

Lemma A.4. If $|A| \subseteq|B|$, then $\boldsymbol{\lambda} k .[\lambda x . x] \in|A \Rightarrow B|$

Proof. From lemma A.3 we have:

$$
\boldsymbol{\lambda} k \cdot[\lambda x . x] \in|A \Rightarrow B| \Leftrightarrow \forall a \in|A|, \boldsymbol{\lambda} k \cdot[x y]\left[\left(\boldsymbol{\lambda} k^{\prime} .[\lambda x . x]\right) k / x, a k / y\right] \in|B|
$$

but since:

$$
\begin{aligned}
\boldsymbol{\lambda} k \cdot[x y]\left[\left(\boldsymbol{\lambda} k^{\prime} \cdot[\lambda x \cdot x]\right) k / x, a k / y\right] & =\boldsymbol{\lambda} k \cdot[x y][[\lambda x \cdot x] / x, a k / y] \\
& =\boldsymbol{\lambda} k \cdot[(\lambda x \cdot x) y][a k / y] \\
& =\boldsymbol{\lambda} k \cdot[y][a k / y] \\
& =\boldsymbol{\lambda} k \cdot y[a k / y] \\
& =\boldsymbol{\lambda} k \cdot a k \\
& =a
\end{aligned}
$$

We have:

$$
\lambda k .[\lambda x . x] \in|A \Rightarrow B| \Leftrightarrow \forall a \in|A|, a \in|B|
$$

from which we conclude using $|A| \subseteq|B|$.

Lemma A.5. If $c \in \llbracket \iota \rrbracket \rightarrow\left[A^{*} \times B^{*}\right]$, then:

$$
\begin{aligned}
& c \in|A \wedge B| \Leftrightarrow \forall a \in|A|, \lambda k \cdot\left[\mathrm{p}_{1}(x)\right][c k / x] \in|A| \\
& \quad \text { and } \forall b \in|B|, \lambda k \cdot\left[\mathrm{p}_{2}(x)\right][c k / x] \in|B|
\end{aligned}
$$


Proof. One one hand:

$$
\begin{aligned}
c \in|A \wedge B| & \Leftrightarrow \quad \forall d \in\|A \wedge B\|, \boldsymbol{\lambda} k \cdot c k(d k) \in \Perp \\
\Leftrightarrow & \forall a \in\|A\|, \boldsymbol{\lambda} k \cdot c k\left(\left(\boldsymbol{\lambda} k^{\prime} \cdot \mathbf{i n}_{1}\left(a k^{\prime}\right)\right) k\right) \in \Perp \\
& \quad \text { and } \forall b \in\|B\|, \boldsymbol{\lambda} k \cdot c k\left(\left(\boldsymbol{\lambda} k^{\prime} \cdot \mathbf{i n}_{2}\left(b k^{\prime}\right)\right) k\right) \in \Perp \\
\Leftrightarrow & \forall a \in\|A\|, \boldsymbol{\lambda} k \cdot c k\left(\mathbf{i n}_{1}(a k)\right) \in \Perp \\
& \quad \text { and } \forall b \in\|B\|, \boldsymbol{\lambda} k \cdot c k\left(\mathbf{i n}_{2}(b k)\right) \in \Perp
\end{aligned}
$$

and on the other hand if $a \in\|A\|$ then:

$$
\begin{aligned}
\boldsymbol{\lambda} k \cdot\left(\boldsymbol{\lambda} k^{\prime} \cdot\left[\mathrm{p}_{1}(x)\right]\left[c k^{\prime} / x\right]\right) k(a k) & =\boldsymbol{\lambda} k \cdot\left(\left[\mathrm{p}_{1}(x)\right][c k / x]\right)(a k) \\
& \left.=\boldsymbol{\lambda} k \cdot\left(\boldsymbol{\lambda} k^{\prime} \cdot x\left(\mathbf{i n}_{1} k^{\prime}\right)\right)[c k / x]\right)(a k) \\
& =\boldsymbol{\lambda} k \cdot\left(\boldsymbol{\lambda} k^{\prime} \cdot c k\left(\mathbf{i n}_{1} k^{\prime}\right)\right)(a k) \\
& =\boldsymbol{\lambda} k \cdot c k\left(\mathbf{i n}_{1}(a k)\right)
\end{aligned}
$$

and similarly, if $b \in\|B\|$ then:

$$
\boldsymbol{\lambda} k \cdot\left(\boldsymbol{\lambda} k^{\prime} \cdot\left[\mathrm{p}_{2}(x)\right]\left[c k^{\prime} / x\right]\right) k(b k)=\boldsymbol{\lambda} k \cdot c k\left(\mathbf{i n}_{2}(2 k)\right)
$$

therefore:

$$
\begin{aligned}
c \in|A \wedge B| \quad \Leftrightarrow \quad \forall a \in\|A\|, \boldsymbol{\lambda} k \cdot\left(\boldsymbol{\lambda} k^{\prime} \cdot\left[\mathrm{p}_{1}(x)\right]\left[c k^{\prime} / x\right]\right) k(a k) \in \Perp \\
\quad \text { and } \forall B \in\|B\|, \boldsymbol{\lambda} k \cdot\left(\boldsymbol{\lambda} k^{\prime} \cdot\left[\mathrm{p}_{2}(x)\right]\left[c k^{\prime} / x\right]\right) k(b k) \in \Perp
\end{aligned}
$$

that is:

$$
c \in|A \wedge B| \Leftrightarrow \lambda k^{\prime} \cdot\left[\mathrm{p}_{1}(x)\right]\left[c k^{\prime} / x\right] \in|A| \text { and } \lambda k^{\prime} \cdot\left[\mathrm{p}_{2}(x)\right]\left[c k^{\prime} / x\right] \in|B|
$$

Lemma A.6. We have $\boldsymbol{\lambda} k .[\lambda x . x] \in \mid e={ }_{\tau}$ el. Moreover,

$$
\begin{array}{cc}
\lambda k \cdot[\lambda x . x] \in\left|\mathrm{k} e f={ }_{\tau} e\right| & \lambda k \cdot[\lambda x . x] \in \mid \mathrm{s} e f g={ }_{\tau} \text { eg }(f g) \mid \\
\lambda k \cdot[\lambda x . x] \in\left|\operatorname{Rec} e f 0={ }_{\tau} e\right| & \lambda k \cdot[\lambda x . x] \in \mid \operatorname{Rec} e f(\mathrm{~S} g)={ }_{\tau} \text { fg }(\operatorname{Rec} e f g) \mid
\end{array}
$$

where in each case, individuals e, $f, g \in \tau^{\mathrm{t}}$ have the appropriate types $\tau$.

Proof. First remark that if $e, f \in \tau^{\mathrm{t}}$ are such that $e=f$, then $\left\|e \neq_{\tau} f\right\|=$ $\{\boldsymbol{\lambda} k . k\}=|| \perp||$, so $\left|e \neq_{\tau} f\right|=|\perp|$. Since $\left|e={ }_{\tau} f\right|$ is $\left|e \neq_{\tau} f \Rightarrow \perp\right|$, we have $\boldsymbol{\lambda} k .[\boldsymbol{\lambda} x . x] \in\left|e=_{\tau} f\right|$ by lemma A.4. All the results of the lemma are then instances of this, using the interpretation of the terms in the model and its adequacy. For example if $e \in \tau^{\mathrm{t}}$ and $f \in \sigma^{\mathrm{t}}$, then:

$$
\left[\mathrm{k}^{\dagger} x y\right][e / x, f / y]=[(\lambda x y . x) x y][e / x, f / y]=[x][e / x, f / y]=x[e / x, f / y]=e
$$

Lemma A.7. $\lambda k \cdot[\lambda x . \lambda y . y x] \in\left|A[e / z] \Rightarrow \neg A[f / z] \Rightarrow e \neq_{\tau} f\right|$ for $e, f \in \tau^{\mathrm{t}}$. 
Proof. From lemma A.3 we have:

$$
\begin{aligned}
& \lambda k \cdot[\lambda x . \lambda y . y x] \in\left|A[e / z] \Rightarrow \neg A[f / z] \Rightarrow e \neq_{\tau} f\right| \\
& \quad \Leftrightarrow \forall a \in|A[e / z]|, \lambda k \cdot\left[x^{\prime} y^{\prime}\right]\left[[\lambda x \cdot \lambda y . y x] / x^{\prime}, a k / y^{\prime}\right] \in\left|\neg A[f / z] \Rightarrow e \neq_{\tau} f\right|
\end{aligned}
$$

but:

$$
\begin{aligned}
\lambda k \cdot\left[x^{\prime} y^{\prime}\right]\left[[\lambda x \cdot \lambda y \cdot y x] / x^{\prime}, a k / y^{\prime}\right] & =\lambda k \cdot\left[(\lambda x \cdot \lambda y \cdot y x) y^{\prime}\right]\left[a k / y^{\prime}\right] \\
& =\lambda k \cdot\left[\lambda y \cdot y y^{\prime}\right]\left[a k / y^{\prime}\right] \\
& =\lambda k \cdot[\lambda y \cdot y x][a k / x]
\end{aligned}
$$

Then we have:

$$
\begin{aligned}
& \lambda k \cdot[\lambda x . \lambda y \cdot y x] \in\left|A[e / z] \Rightarrow \neg A[f / z] \Rightarrow e \neq_{\tau} f\right| \\
& \quad \Leftrightarrow \forall a \in|A[e / z]|, \lambda k \cdot[\lambda y \cdot y x][a k / x] \in\left|\neg A[f / z] \Rightarrow e \neq_{\tau} f\right| \\
& \quad \Leftrightarrow \forall a \in|A[e / z]|, \forall b \in|\neg A[f / z]|, \lambda k \cdot\left[x^{\prime} y^{\prime}\right]\left[[\lambda y \cdot y x][a k / x] / x^{\prime}, b k / y^{\prime}\right] \in\left|e \neq_{\tau} f\right|
\end{aligned}
$$

but:

$$
\begin{aligned}
\lambda k \cdot\left[x^{\prime} y^{\prime}\right]\left[[\lambda y \cdot y x][a k / x] / x^{\prime}, b k / y^{\prime}\right] & =\boldsymbol{\lambda} k \cdot\left[(\lambda y \cdot y x) y^{\prime}\right]\left[a k / x, b k / y^{\prime}\right] \\
& =\boldsymbol{\lambda} k \cdot\left[y^{\prime} x\right]\left[a k / x, b k / y^{\prime}\right] \\
& =\boldsymbol{\lambda} k \cdot[y x][a k / x, b k / y] \\
& =\boldsymbol{\lambda} k \cdot[x y][b k / x, a k / y]
\end{aligned}
$$

therefore:

$$
\begin{aligned}
\lambda k \cdot[\lambda x . \lambda y \cdot y x] \in \mid A[e / z] & \Rightarrow \neg A[f / z] \Rightarrow e \neq_{\tau} f \mid \\
\Leftrightarrow \forall a \in|A[e / z]|, \forall b & \in|\neg A[f / z]|, \lambda k \cdot[x y][b k / x, a k / y] \in\left|e \neq_{\tau} f\right|
\end{aligned}
$$

If $e \neq f$, then $\left|e \neq_{\tau} f\right|=\llbracket \iota \rrbracket \rightarrow[\iota]$ and we are done. If $e=f$, then $|\neg A[f / z]|=$ $|\neg A[e / z]|=|A[e / z] \Rightarrow \perp|$, so by lemma A.3, if $a \in|A[e / z]|$ and $b \in|\neg A[f / z]|$, then $\boldsymbol{\lambda} k \cdot[x y][b k / x, a k / y] \in|\perp|=\left|e \neq_{\tau} f\right|($ since $e=f)$.

Lemma A.8. If e, $f \in \mathcal{I}_{0}^{\tau}$ are closed first order terms with parameters such that $\left[e^{\dagger}\right]=\left[f^{\dagger}\right]$, then for any formula $A$ with $F V(A)=\left\{x^{\tau}\right\}$, we have $\|A[e / x]\|=$ $\|A[f / x]\|$.

Proof. We first prove that for any $g \in \mathcal{I}^{\sigma}$ with parameters and $\operatorname{FV}(g)=\left\{x^{\tau}\right\}$, we have $\left[g[e / x]^{\dagger}\right]=\left[g[f / x]^{\dagger}\right]$ by induction on the structure of the term $g$. Then the proof goes by induction on the structure of the formula $A$.

Lemma A.9. (i) For all $n \in \mathbb{N}$ and all $e \in \iota^{\mathrm{t}}$, we have $\boldsymbol{\lambda} k \cdot[\bar{n}] \in \mid \mathrm{S} e \neq \iota$ 이.

(ii) $\lambda k \cdot[\lambda x \cdot \lambda y \cdot \operatorname{rec}(x, y)] \in\left|A[0 / x] \Rightarrow \forall x^{\iota}(A \Rightarrow A[\mathrm{~S} x / x]) \Rightarrow \forall x^{\iota} A\right|$.

Proof. (i) Since for any $e \in \iota^{\mathrm{t}}$ there is some $m \in \mathbb{N}$ such that $e=[\bar{m}]$, we have:

$$
\begin{aligned}
{\left[\mathrm{S}^{\dagger} x\right][e / x] } & =\left[\mathrm{S}^{\dagger} x\right][[\bar{n}] / x] \\
& =\left[\mathrm{S}^{\dagger} \bar{n}\right] \\
& =[\operatorname{succ} \bar{n}] \\
& =[n+1]
\end{aligned}
$$


so $\left[\mathrm{S}^{\dagger} x\right][e / x]=[\overline{n+1}] \neq[\overline{0}]=\left[0^{\dagger}\right]$. Therefore $\| \mathrm{S} e \neq \iota 0||=\emptyset$, so $|\mathrm{S} e \neq \iota 0|=$ $\llbracket \iota \rrbracket \rightarrow[\iota]$, and therefore for any $n \in \mathbb{N}$ we have $\lambda k \cdot[\bar{n}] \in \mid \mathrm{S} e \neq \iota$ o|.

(ii) We have by lemmas A.3 and A.1:

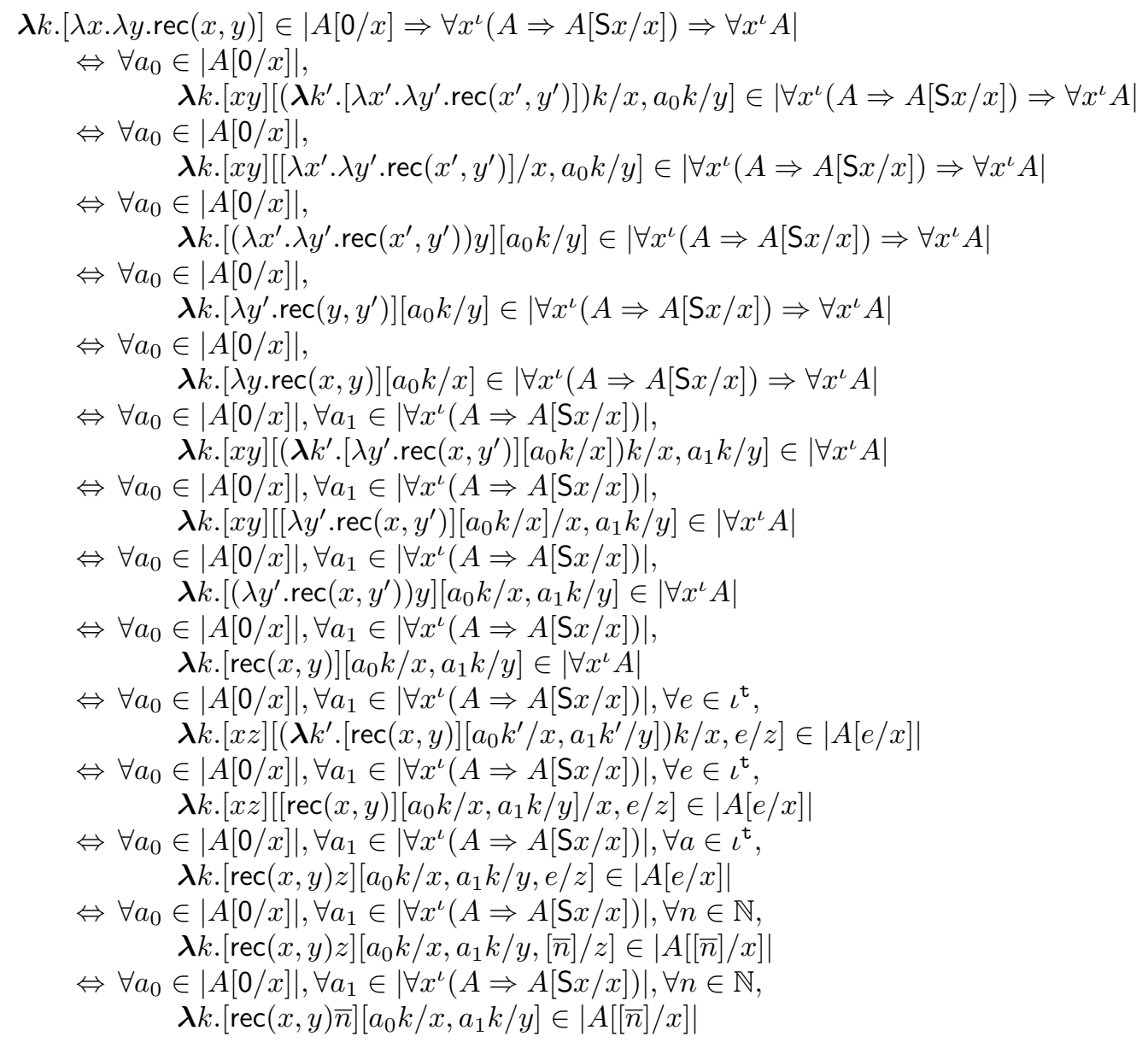

Since for all $e \in \iota^{\mathrm{t}}$ there is some $n \in \mathbb{N}$ such that $e=[\bar{n}]$, and therefore $|A[e / x]|=|A[[\bar{n}] / x]|$. Let us fix $a_{0} \in|A[0 / x]|$ and $a_{1} \in\left|\forall x^{\iota}(A \Rightarrow A[\mathrm{~S} x / x])\right|$. We have:

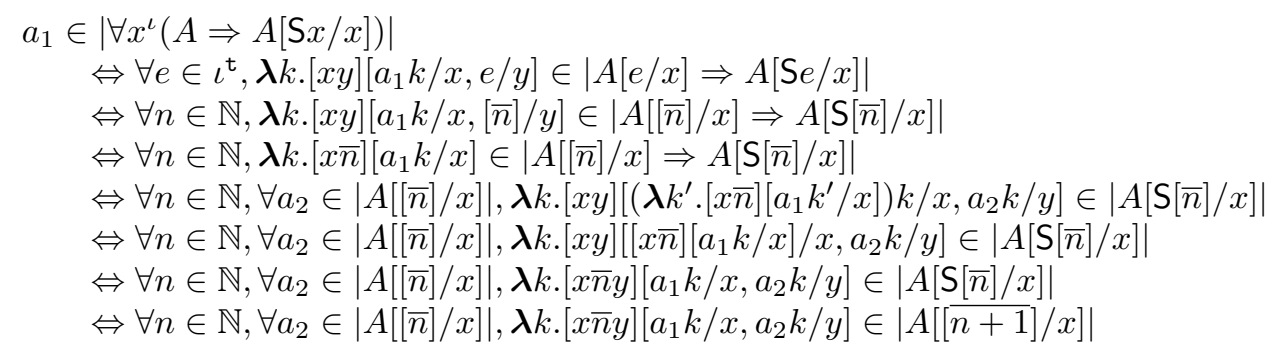


by lemma A.8, since $\left[(\mathrm{S}[\bar{n}])^{\dagger}\right]=\left[(\mathrm{S} x)^{\dagger}\right][[\bar{n}] / x]=[\operatorname{succ} x][[\bar{n}] / x]=[\operatorname{succ} \bar{n}]=$ $[\overline{n+1}]$. We now prove by induction on $n \in \mathbb{N}$ that:

$$
\lambda k \cdot[\operatorname{rec}(x, y) \bar{n}]\left[a_{0} k / x, a_{1} k / y\right] \in|A[[\bar{n}] / x]|
$$

$-n=0$ :

$$
\begin{aligned}
\lambda k \cdot[\operatorname{rec}(x, y) \overline{0}]\left[a_{0} k / x, a_{1} k / y\right] & =\lambda k \cdot[x]\left[a_{0} k / x, a_{1} k / y\right] \\
& =\lambda k \cdot x\left[a_{0} k / x, a_{1} k / y\right] \\
& =\lambda k \cdot a_{0} k \\
& =a_{0} \in|A[0 / x]|=\left|A\left[\left[0^{\dagger}\right] / x\right]\right|=|A[[\overline{0}] / x]|
\end{aligned}
$$

$-n=m+1:$ the induction hypothesis gives:

$$
\boldsymbol{\lambda} k \cdot[\operatorname{rec}(x, y) \bar{m}]\left[a_{0} k / x, a_{1} k / y\right] \in|A[[\bar{m}] / x]|
$$

and $a_{1}$ is such that:

$$
\forall a_{2} \in|A[[\bar{m}] / x]|, \boldsymbol{\lambda} k \cdot[x \bar{m} y]\left[a_{1} k / x, a_{2} k / y\right] \in|A[[\overline{m+1}] / x]|
$$

therefore:

$$
\boldsymbol{\lambda} k \cdot[x \bar{m} y]\left[a_{1} k / x,\left(\boldsymbol{\lambda} k^{\prime} \cdot\left[\operatorname{rec}\left(x^{\prime}, y^{\prime}\right) \bar{m}\right]\left[a_{0} k^{\prime} / x^{\prime}, a_{1} k^{\prime} / y^{\prime}\right]\right) k / y\right] \in|A[[\overline{m+1}] / x]|
$$

but:

$$
\begin{aligned}
\boldsymbol{\lambda} k \cdot[ & x \bar{m} y]\left[a_{1} k / x,\left(\boldsymbol{\lambda} k^{\prime} \cdot\left[\operatorname{rec}\left(x^{\prime}, y^{\prime}\right) \bar{m}\right]\left[a_{0} k^{\prime} / x^{\prime}, a_{1} k^{\prime} / y^{\prime}\right]\right) k / y\right] \\
& =\boldsymbol{\lambda} k \cdot[x \bar{m} y]\left[a_{1} k / x,\left[\operatorname{rec}\left(x^{\prime}, y^{\prime}\right) \bar{m}\right]\left[a_{0} k / x^{\prime}, a_{1} k / y^{\prime}\right] / y\right] \\
& =\lambda k \cdot\left[x \bar{m}\left(\operatorname{rec}\left(x^{\prime}, y^{\prime}\right) \bar{m}\right)\right]\left[a_{1} k / x, a_{0} k / x^{\prime}, a_{1} k / y^{\prime}\right] \\
& =\boldsymbol{\lambda} k \cdot\left[y^{\prime} \bar{m}\left(\operatorname{rec}\left(x^{\prime}, y^{\prime}\right) \bar{m}\right)\right]\left[a_{0} k / x^{\prime}, a_{1} k / y^{\prime}\right] \\
& =\boldsymbol{\lambda} k \cdot[y \bar{m}(\operatorname{rec}(x, y) \bar{m})]\left[a_{0} k / x, a_{1} k / y\right] \\
& =\boldsymbol{\lambda} k \cdot[\operatorname{rec}(x, y) \overline{m+1})]\left[a_{0} k / x, a_{1} k / y\right]
\end{aligned}
$$

so we conclude:

$$
\boldsymbol{\lambda} k \cdot[\operatorname{rec}(x, y) \bar{n})]\left[a_{0} k / x, a_{1} k / y\right] \in|A[[\bar{n}] / x]|
$$

We associate to each axiom $A$ a closed typed $\lambda \mu$-term $\xi_{A}$ as follows:

$$
\begin{aligned}
& \xi_{\forall x^{\tau}\left(x={ }_{\tau} x\right)}=\lambda x y \cdot y: \tau \rightarrow \iota \rightarrow \iota \\
& \xi_{\forall x^{\tau} \forall y^{\tau}\left(A[x] \Rightarrow \neg A[y] \Rightarrow x \neq_{\tau} y\right)}=\lambda x y u v . v u: \tau \rightarrow \tau \rightarrow A^{*} \rightarrow\left(A^{*} \rightarrow \iota\right) \rightarrow \iota \\
& \left.\xi_{\forall x^{\tau} \forall y^{\sigma}\left(\mathrm{k} x y={ }_{\tau} x\right)}=\lambda x y u . u: \tau \rightarrow \sigma \rightarrow \iota \rightarrow \iota\right) \rightarrow \iota \\
& \xi_{\forall x^{\tau} \forall y^{\sigma} \forall z^{\nu}\left(\operatorname{sxyz}={ }_{\nu} x z(y z)\right)}=\lambda x y z u . u: \tau \rightarrow \sigma \rightarrow \nu \rightarrow \iota \rightarrow \iota \\
& \xi_{\forall x^{\iota}(S x \neq \iota)}=\lambda x \cdot \overline{0}: \iota \rightarrow \iota \\
& \xi_{\forall x^{\tau} \forall y^{\iota \rightarrow \tau \rightarrow \tau}\left(\operatorname{Rec} x y 0={ }_{\tau} x\right)}=\lambda x y u . u: \tau \rightarrow(\iota \rightarrow \tau \rightarrow \tau) \rightarrow \iota \rightarrow \iota \\
& \xi_{\forall x^{\tau} \forall y^{\iota \rightarrow \tau \rightarrow \tau} \forall z^{\iota}\left(\operatorname{Rec} x y \mathrm{~S} z={ }_{\tau} y z(\operatorname{Rec} x y z)\right)}=\lambda x y z u . u: \tau \rightarrow(\iota \rightarrow \tau \rightarrow \tau) \rightarrow \iota \rightarrow \iota \rightarrow \iota \\
& \xi_{A[0]} \Rightarrow \forall y^{\tau}(A[y] \Rightarrow A[\mathrm{~S} y]) \Rightarrow \forall x^{\iota} A[x] \quad=\quad \lambda u v \cdot \operatorname{rec}(u, v): A^{*} \rightarrow\left(\iota \rightarrow A^{*} \rightarrow A^{*}\right) \rightarrow \iota \rightarrow A^{*}
\end{aligned}
$$

We extend the translation $\left({ }_{-}\right)^{*}$ to contexts in the obvious way: $\left(A_{1}, \ldots, A_{n}\right)^{*}$ is translated to $x_{1}: A_{1}{ }^{*}, \ldots, x_{n}: A_{n}{ }^{*}$. 
We translate each derivation $\frac{\varepsilon}{\Gamma \vdash A_{\varepsilon} \Delta}$ to a typing derivation of $\boldsymbol{x}: \boldsymbol{\tau}, \Gamma^{*} \vdash$ $\varepsilon^{*}: A^{*} \mid \Delta^{*}$ and each derivation $\frac{\varepsilon}{(\Gamma \vdash \Delta)}$ to a typing derivation of $\varepsilon^{*}:(\boldsymbol{x}:$ $\left.\boldsymbol{\tau}, \Gamma^{*} \vdash \Delta^{*}\right)$, where the free variables $x^{\tau}$ of $\Gamma, A, \Delta$ occur as $x: \tau$ in $\boldsymbol{x}: \boldsymbol{\tau}$ :

$$
\left(\frac{}{\Gamma, A \vdash A \mid \Delta}\right)^{*}=\frac{\boldsymbol{x}: \boldsymbol{\tau}, \Gamma^{*}, x: A^{*} \vdash x: A^{*} \mid \Delta^{*}}{\text { where } \boldsymbol{x}} \quad=\quad \operatorname{FV}(\Gamma, A, \Delta)
$$$$
\left(\frac{\vdots}{\Gamma \vdash A \mid \Delta}(A \text { axiom })\right)^{*}=\frac{\vdots}{\boldsymbol{x}: \boldsymbol{\tau}, \Gamma^{*} \vdash \xi_{A}: A^{*} \mid \Delta^{*}} \quad \text { where } \boldsymbol{x}=\operatorname{FV}(\Gamma, \Delta)
$$$$
\left(\frac{\frac{\varepsilon}{\Gamma \vdash \perp^{\prime} \Delta}}{\Gamma \vdash a^{\tau} \neq_{\tau} b^{\tau} \mid \Delta}\right)^{*}=\frac{\vdots}{\boldsymbol{x}: \boldsymbol{\tau}, \boldsymbol{y}: \boldsymbol{\sigma}, \Gamma^{*} \vdash \varepsilon^{*}: \iota \mid \Delta^{*}} \quad \text { where } \boldsymbol{y}=\mathrm{FV}\left(a^{\tau}, b^{\tau}\right)
$$

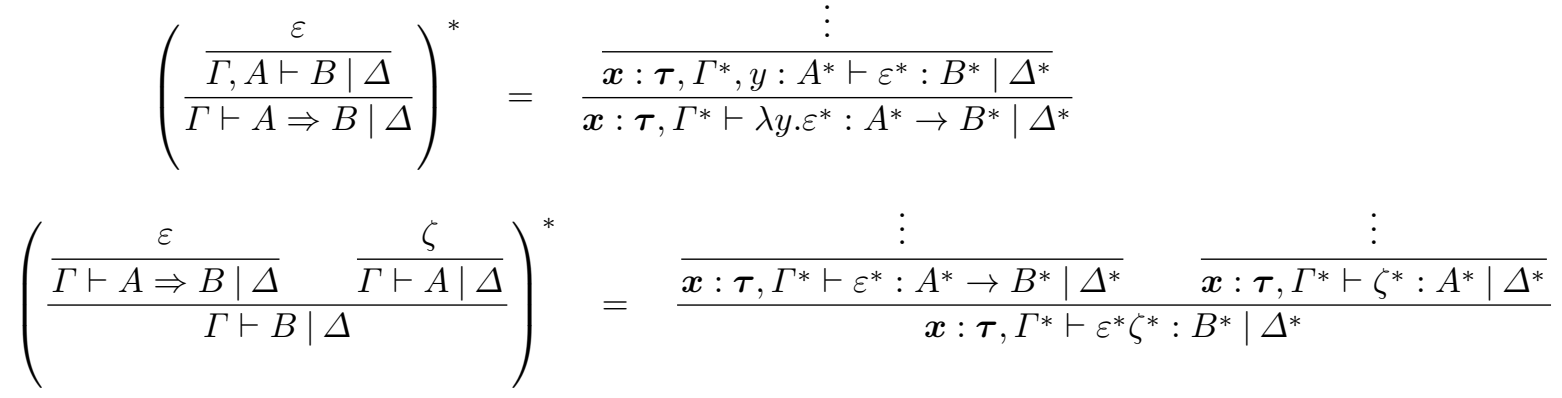$$
\left(\frac{\frac{\varepsilon}{\Gamma \vdash A \mid \Delta} \frac{\zeta}{\Gamma \vdash B \mid \Delta}}{\Gamma \vdash A \wedge B \mid \Delta}\right)^{*}=\frac{\frac{\vdots}{x: \tau, \Gamma^{*} \vdash \varepsilon^{*}: A^{*} \mid \Delta^{*}}}{\boldsymbol{x}: \boldsymbol{\tau}, \Gamma^{*} \vdash\left\langle\varepsilon^{*}, \zeta^{*}\right\rangle: A^{*} \times B^{*} \mid \Delta^{*}}
$$$$
\left(\frac{\frac{\varepsilon}{\Gamma \vdash A_{1} \wedge A_{2} \mid \Delta}}{\Gamma \vdash A_{i} \mid \Delta}(i=1,2)\right)^{*}=\frac{\vdots}{\frac{\boldsymbol{x}: \boldsymbol{\tau}, \Gamma^{*} \vdash \varepsilon^{*}: A^{*} \times B^{*} \mid \Delta^{*}}{\boldsymbol{x}: \boldsymbol{\tau}, \Gamma^{*} \vdash \mathrm{p}_{i}\left(\varepsilon^{*}\right): A_{i}^{*} \mid \Delta^{*}}}
$$$$
\left(\frac{\frac{\varepsilon}{\Gamma \vdash A \mid \Delta}}{\Gamma \vdash \forall x^{\tau} A \mid \Delta}(x \notin \mathrm{FV}(\Gamma, \Delta))\right)^{*}=\frac{\frac{\vdots}{\boldsymbol{x}: \boldsymbol{\tau}, x: \tau, \Gamma^{*} \vdash \varepsilon^{*}: A^{*} \mid \Delta^{*}}}{\boldsymbol{x}: \boldsymbol{\tau}, \Gamma^{*} \vdash \lambda x . \varepsilon^{*}: \tau \rightarrow A^{*} \mid \Delta^{*}}
$$$$
\left(\frac{\varepsilon}{\Gamma \vdash \forall x^{\tau} A \mid \Delta} \frac{\vdots}{\Gamma \vdash A\left[a^{\tau} / x\right] \mid \Delta}\right)^{*}=\frac{\frac{\boldsymbol{x}: \boldsymbol{\tau}, \boldsymbol{y}: \boldsymbol{\sigma}, \Gamma^{*} \vdash \varepsilon^{*}: \tau \rightarrow A^{*} \mid \Delta^{*}}{\boldsymbol{x}: \boldsymbol{\tau}, \boldsymbol{y}: \boldsymbol{\sigma}, \Gamma^{*} \vdash \varepsilon^{*} a^{\tau \dagger}: A^{*} \mid \Delta^{*}}}{\text { where } \boldsymbol{y}}=\operatorname{FV}\left(a^{\tau}\right)
$$ 


$$
\begin{aligned}
& \left(\frac{\frac{\varepsilon}{\Gamma \vdash A \mid \Delta, A}}{(\Gamma \vdash \Delta, A)}\right)^{*}=\frac{\frac{\vdots}{\boldsymbol{x}: \boldsymbol{\tau}, \Gamma^{*} \vdash \varepsilon^{*}: A^{*} \mid \Delta^{*}, \alpha: A^{*}}}{[\alpha] \varepsilon^{*}:\left(\boldsymbol{x}: \boldsymbol{\tau}, \Gamma^{*} \vdash \Delta^{*}, \alpha: A^{*}\right)}
\end{aligned}
$$

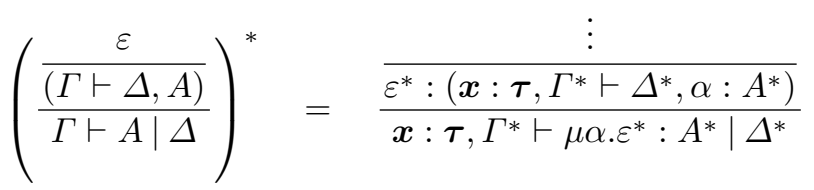

Theorem A.10. Let $\frac{\varepsilon}{\Gamma \vdash A \mid \Delta}$ in $\mathrm{PA}^{\omega}$, with $F V(\Gamma, A, \Delta) \subseteq\left\{x_{1}^{\tau_{1}}, \ldots, x_{k}^{\tau_{k}}\right\}$, $\Gamma=A_{1}, \ldots, A_{n}$ and $\Delta=B_{1}, \ldots, B_{m}$.

Then

$$
x_{1}: \tau_{1}, \ldots, x_{k}: \tau_{k}, y_{1}: A_{1}{ }^{*}, \ldots, y_{n}: A_{n}{ }^{*} \vdash \varepsilon^{*}: A^{*} \mid \alpha_{1}: B_{1}{ }^{*}, \ldots, \alpha_{m}: B_{m}{ }^{*}
$$

is such that for all $c_{1} \in \tau_{1}{ }^{\mathrm{t}}, \ldots, c_{k} \in \tau_{k}{ }^{\mathrm{t}}$, all $a_{1} \in\left|A_{1}[\boldsymbol{c} / \boldsymbol{x}]\right|, \ldots, a_{n} \in\left|A_{n}[\boldsymbol{c} / \boldsymbol{x}]\right|$, and all $b_{1} \in\left\|B_{1}[\boldsymbol{c} / \boldsymbol{x}]\right\|, \ldots, b_{m} \in\left\|B_{m}[\boldsymbol{c} / \boldsymbol{x}]\right\|$, we have

$$
\boldsymbol{\lambda} k \cdot\left[\varepsilon^{*}\right][\boldsymbol{c} / \boldsymbol{x}, \boldsymbol{a} k / \boldsymbol{y}, \boldsymbol{b} k / \boldsymbol{\alpha}] \in|A[\boldsymbol{c} / \boldsymbol{x}]|
$$

Proof. By induction on the structure of the derivation:

$-\varepsilon=\overline{\Gamma, A \vdash A \mid \Delta}:$

$\boldsymbol{\lambda} k .\left[\varepsilon^{*}\right][\boldsymbol{c} / \boldsymbol{x}, \boldsymbol{a} k / \boldsymbol{y}, a k / x, \boldsymbol{b} k / \boldsymbol{\alpha}]=\boldsymbol{\lambda} k .[x][a k / x]=\boldsymbol{\lambda} k . a k=a \in|A[\boldsymbol{c} / \boldsymbol{x}]|$

$-\varepsilon=\frac{}{\Gamma \vdash A \mid \Delta}(A$ axiom $)$ : then by lemmas A.6 A.7 A.9 we have $\lambda k \cdot\left[\varepsilon^{*}\right]=$ $\boldsymbol{\lambda} k .\left[\xi_{A}\right] \in|A|=|A[\boldsymbol{c} / \boldsymbol{x}]|$ since $A$ is closed. Moreover, since $\xi_{A}$ is closed,

$$
\boldsymbol{\lambda} k \cdot\left[\varepsilon^{*}\right][\boldsymbol{c} / \boldsymbol{x}, \boldsymbol{a} k / \boldsymbol{y}, \boldsymbol{b} k / \boldsymbol{\alpha}]=\boldsymbol{\lambda} k \cdot\left[\varepsilon^{*}\right] \in|A[\boldsymbol{c} / \boldsymbol{x}]|
$$

$-\varepsilon=\frac{\varepsilon^{\prime}}{\Gamma \vdash \perp \mid \Delta} \frac{1}{\Gamma \vdash a^{\tau} \neq_{\tau} b^{\tau} \mid \Delta}$ : this comes from the fact that since $\left\|a \neq_{\tau} b\right\| \subseteq$ $\{\boldsymbol{\lambda} k . k\}=\left\|\underset{\varepsilon^{\prime}}{\Perp}\right\|$, we have $|\Perp| \subseteq\left|a \neq_{\tau} b\right|$

$-\varepsilon=\frac{\overline{\Gamma, A \vdash B \mid \Delta}}{\Gamma \vdash A \Rightarrow B \mid \Delta}$ : the induction hypothesis gives for any $c_{1} \in \tau_{1}{ }^{\mathrm{t}}, \ldots, c_{k} \in$ $\tau_{k}{ }^{\mathrm{t}}, a_{1} \in\left|A_{1}[\boldsymbol{c} / \boldsymbol{x}]\right|, \ldots, a_{n} \in\left|A_{n}[\boldsymbol{c} / \boldsymbol{x}]\right|, b_{1} \in\left\|B_{1}[\boldsymbol{c} / \boldsymbol{x}]\right\|, \ldots, b_{m} \in\left\|B_{m}[\boldsymbol{c} / \boldsymbol{x}]\right\|:$

$$
\forall a \in|A[\boldsymbol{c} / \boldsymbol{x}]|, \boldsymbol{\lambda} k \cdot\left[\varepsilon^{\prime *}\right][\boldsymbol{c} / \boldsymbol{x}, \boldsymbol{a} k / \boldsymbol{y}, a k / y, \boldsymbol{b} k / \boldsymbol{\alpha}] \in|B[\boldsymbol{c} / \boldsymbol{x}]|
$$

using lemma A.3, in order to prove:

$$
\boldsymbol{\lambda} k \cdot\left[\lambda y \cdot \varepsilon^{\prime *}\right][\boldsymbol{c} / \boldsymbol{x}, \boldsymbol{a} k / \boldsymbol{y}, \boldsymbol{b} k / \boldsymbol{\alpha}] \in|A[\boldsymbol{c} / \boldsymbol{x}] \Rightarrow B[\boldsymbol{c} / \boldsymbol{x}]|
$$


it suffices to prove:

$\forall a \in|A[\boldsymbol{c} / \boldsymbol{x}]|, \boldsymbol{\lambda} k \cdot[x y]\left[\left(\boldsymbol{\lambda} k^{\prime} \cdot\left[\lambda y \cdot \varepsilon^{\prime *}\right]\left[\boldsymbol{c} / \boldsymbol{x}, \boldsymbol{a} k^{\prime} / \boldsymbol{y}, \boldsymbol{b} k^{\prime} / \boldsymbol{\alpha}\right]\right) k / x, a k / y\right] \in|B[\boldsymbol{c} / \boldsymbol{x}]|$ but if $a \in|A[\boldsymbol{c} / \boldsymbol{x}]|$, then:

$$
\begin{aligned}
& \boldsymbol{\lambda} k \cdot[x y]\left[\left(\boldsymbol{\lambda} k^{\prime} \cdot\left[\lambda y \cdot \varepsilon^{\prime *}\right]\left[\boldsymbol{c} / \boldsymbol{x}, \boldsymbol{a}\left(k^{\prime}\right) / \boldsymbol{y}, \boldsymbol{b}\left(k^{\prime}\right) / \boldsymbol{\alpha}\right]\right) k / x, a k / y\right] \\
&=\boldsymbol{\lambda} k \cdot[x y]\left[\left[\lambda y \cdot \varepsilon^{\prime *}\right][\boldsymbol{c} / \boldsymbol{x}, \boldsymbol{a} k / \boldsymbol{y}, \boldsymbol{b} k / \boldsymbol{\alpha}] / x, a k / y\right] \\
&=\boldsymbol{\lambda} k \cdot\left[\left(\lambda y \cdot \varepsilon^{\prime *}\right) y\right][\boldsymbol{c} / \boldsymbol{x}, \boldsymbol{a} k / \boldsymbol{y}, \boldsymbol{b} k / \boldsymbol{\alpha}, a k / y] \\
&=\boldsymbol{\lambda} k \cdot\left[\left(\lambda y \cdot \varepsilon^{*}\right) y\right][\boldsymbol{c} / \boldsymbol{x}, \boldsymbol{a} k / \boldsymbol{y}, \boldsymbol{b} k / \boldsymbol{\alpha}, a k / y] \\
&=\boldsymbol{\lambda} k \cdot\left[\varepsilon^{\prime *}\right][\boldsymbol{c} / \boldsymbol{x}, \boldsymbol{a} k / \boldsymbol{y}, \boldsymbol{b} k / \boldsymbol{\alpha}, a k / y]
\end{aligned}
$$

so we conclude using the induction hypothesis.

$$
\begin{gathered}
-\varepsilon=\frac{\frac{\varepsilon^{\prime}}{\Gamma \vdash A \Rightarrow B \mid \Delta} \quad \frac{\zeta}{\Gamma \vdash A \mid \Delta}}{\Gamma \vdash B \mid \Delta}: \text { let } c_{1} \in \tau_{1}{ }^{\mathrm{t}}, \ldots, c_{k} \in \tau_{k}{ }^{\mathrm{t}}, a_{1} \in\left|A_{1}[\boldsymbol{c} / \boldsymbol{x}]\right|, \\
\ldots, a_{n} \in\left|A_{n}[\boldsymbol{c} / \boldsymbol{x}]\right|, b_{1} \in\left\|B_{1}[\boldsymbol{c} / \boldsymbol{x}]\right\|, \ldots, b_{m} \in\left\|B_{m}[\boldsymbol{c} / \boldsymbol{x}]\right\| . \text { By induction }
\end{gathered}
$$
hypothesis we have:

$$
\boldsymbol{\lambda} k \cdot\left[\varepsilon^{\prime *}\right][\boldsymbol{c} / \boldsymbol{x}, \boldsymbol{a} k / \boldsymbol{y}, \boldsymbol{b} k / \boldsymbol{\alpha}] \in|A[\boldsymbol{c} / \boldsymbol{x}] \Rightarrow B[\boldsymbol{c} / \boldsymbol{x}]|
$$

so by lemma A.3 we get:

$$
\forall a \in|A[\boldsymbol{c} / \boldsymbol{x}]|, \boldsymbol{\lambda} k \cdot[x y]\left[\left(\boldsymbol{\lambda} k^{\prime} \cdot\left[\varepsilon^{\prime *}\right]\left[\boldsymbol{c} / \boldsymbol{x}, \boldsymbol{a} k^{\prime} / \boldsymbol{y}, \boldsymbol{b} k^{\prime} / \boldsymbol{\alpha}\right]\right) k / x, a k / y\right] \in|B[\boldsymbol{c} / \boldsymbol{x}]|
$$

but:

$$
\begin{gathered}
\boldsymbol{\lambda} k \cdot[x y]\left[\left(\boldsymbol{\lambda} k^{\prime} \cdot\left[\varepsilon^{\prime *}\right]\left[\boldsymbol{c} / \boldsymbol{x}, \boldsymbol{a} k^{\prime} / \boldsymbol{y}, \boldsymbol{b} k^{\prime} / \boldsymbol{\alpha}\right]\right) k / x, a k / y\right] \\
=\boldsymbol{\lambda} k \cdot[x y]\left[\left[\varepsilon^{\prime *}\right][\boldsymbol{c} / \boldsymbol{x}, \boldsymbol{a} k / \boldsymbol{y}, \boldsymbol{b} k / \boldsymbol{\alpha}] / x, a k / y\right] \\
=\boldsymbol{\lambda} k \cdot\left[\varepsilon^{\prime *} y\right][\boldsymbol{c} / \boldsymbol{x}, \boldsymbol{a} k / \boldsymbol{y}, \boldsymbol{b} k / \boldsymbol{\alpha}, a k / y]
\end{gathered}
$$

and since again by induction hypothesis we have:

$$
\boldsymbol{\lambda} k \cdot\left[\zeta^{*}\right][\boldsymbol{c} / \boldsymbol{x}, \boldsymbol{a} k / \boldsymbol{y}, \boldsymbol{b} k / \boldsymbol{\alpha}] \in|A[\boldsymbol{c} / \boldsymbol{x}]|
$$

we get:

$$
\boldsymbol{\lambda} k .\left[\varepsilon^{\prime *} y\right]\left[\boldsymbol{c} / \boldsymbol{x}, \boldsymbol{a} k / \boldsymbol{y}, \boldsymbol{b} k / \boldsymbol{\alpha},\left(\boldsymbol{\lambda} k^{\prime} \cdot\left[\zeta^{*}\right]\left[\boldsymbol{c} / \boldsymbol{x}, \boldsymbol{a} k^{\prime} / \boldsymbol{y}, \boldsymbol{b} k^{\prime} / \boldsymbol{\alpha}\right]\right) k / y\right] \in|B[\boldsymbol{c} / \boldsymbol{x}]|
$$

so we can conclude since:

$$
\begin{aligned}
& \boldsymbol{\lambda} k .\left[\varepsilon^{\prime *} y\right]\left[\boldsymbol{c} / \boldsymbol{x}, \boldsymbol{a} k / \boldsymbol{y}, \boldsymbol{b} k / \boldsymbol{\alpha},\left(\boldsymbol{\lambda} k^{\prime} .\left[\zeta^{*}\right]\left[\boldsymbol{c} / \boldsymbol{x}, \boldsymbol{a} k^{\prime} / \boldsymbol{y}, \boldsymbol{b} k^{\prime} / \boldsymbol{\alpha}\right]\right) k / y\right] \\
& =\lambda k \cdot\left[\varepsilon^{\prime *} y\right]\left[\boldsymbol{c} / \boldsymbol{x}, \boldsymbol{a} k / \boldsymbol{y}, \boldsymbol{b} k / \boldsymbol{\alpha},\left[\zeta^{*}\right][\boldsymbol{c} / \boldsymbol{x}, \boldsymbol{a} k / \boldsymbol{y}, \boldsymbol{b} k / \boldsymbol{\alpha}] / y\right] \\
& =\boldsymbol{\lambda} k \cdot\left[\varepsilon^{\prime *} \zeta^{*}\right][\boldsymbol{c} / \boldsymbol{x}, \boldsymbol{a} k / \boldsymbol{y}, \boldsymbol{b} k / \boldsymbol{\alpha}] \\
& -\varepsilon=\frac{\frac{\varepsilon^{\prime}}{\Gamma \vdash A \mid \Delta} \quad \frac{\zeta}{\Gamma \vdash B \mid \Delta}}{\Gamma \vdash A \wedge B \mid \Delta} \text { : by induction hypothesis we have: }
\end{aligned}
$$

$$
\boldsymbol{\lambda} k \cdot\left[\varepsilon^{\prime *}\right][\boldsymbol{c} / \boldsymbol{x}, \boldsymbol{a} k / \boldsymbol{y}, \boldsymbol{b} k / \boldsymbol{\alpha}] \in|A[\boldsymbol{c} / \boldsymbol{x}]|
$$


and

$$
\boldsymbol{\lambda} k \cdot\left[\zeta^{*}\right][\boldsymbol{c} / \boldsymbol{x}, \boldsymbol{a} k / \boldsymbol{y}, \boldsymbol{b} k / \boldsymbol{\alpha}] \in|B[\boldsymbol{c} / \boldsymbol{x}]|
$$

Using lemma A.5, we have:

$$
\begin{aligned}
\boldsymbol{\lambda} k \cdot\left[\left\langle\varepsilon^{\prime *}, \zeta^{*}\right\rangle\right][\boldsymbol{c} / \boldsymbol{x}, \boldsymbol{a} k / \boldsymbol{y}, \boldsymbol{b} k / \boldsymbol{\alpha}] \in|A[\boldsymbol{c} / \boldsymbol{x}] \wedge B[\boldsymbol{c} / \boldsymbol{x}]| \\
\Leftrightarrow \boldsymbol{\lambda} k \cdot\left[\mathrm{p}_{1}(x)\right]\left[\left(\boldsymbol{\lambda} k^{\prime} \cdot\left[\left\langle\varepsilon^{\prime *}, \zeta^{*}\right\rangle\right]\left[\boldsymbol{c} / \boldsymbol{x}, \boldsymbol{a} k^{\prime} / \boldsymbol{y}, \boldsymbol{b} k^{\prime} / \boldsymbol{\alpha}\right]\right) k / x\right] \in A[\boldsymbol{c} / \boldsymbol{x}]^{*} \\
\quad \text { and } \boldsymbol{\lambda} k \cdot\left[\mathrm{p}_{2}(x)\right]\left[\left(\boldsymbol{\lambda} k^{\prime} \cdot\left[\left\langle\varepsilon^{\prime *}, \zeta^{*}\right\rangle\right]\left[\boldsymbol{c} / \boldsymbol{x}, \boldsymbol{a} k^{\prime} / \boldsymbol{y}, \boldsymbol{b} k^{\prime} / \boldsymbol{\alpha}\right]\right) k / x\right] \in B[\boldsymbol{c} / \boldsymbol{x}]^{*} \\
\left.\Leftrightarrow \boldsymbol{\lambda} k \cdot\left[\mathrm{p}_{1}(x)\right]\left[\left\langle\varepsilon^{\prime *}, \zeta^{*}\right\rangle\right][\boldsymbol{c} / \boldsymbol{x}, \boldsymbol{a} k / \boldsymbol{y}, \boldsymbol{b} k / \boldsymbol{\alpha}] / x\right] \in A[\boldsymbol{c} / \boldsymbol{x}]^{*} \\
\quad \text { and } \boldsymbol{\lambda} k \cdot\left[\mathrm{p}_{2}(x)\right]\left[\left[\left\langle\varepsilon^{\prime *}, \zeta^{*}\right\rangle\right][\boldsymbol{c} / \boldsymbol{x}, \boldsymbol{a} k / \boldsymbol{y}, \boldsymbol{b} k / \boldsymbol{\alpha}] / x\right] \in B[\boldsymbol{c} / \boldsymbol{x}]^{*} \\
\Leftrightarrow \boldsymbol{\lambda} k \cdot\left[\mathrm{p}_{1}\left(\left\langle\varepsilon^{\prime *}, \zeta^{*}\right\rangle\right)\right][\boldsymbol{c} / \boldsymbol{x}, \boldsymbol{a} k / \boldsymbol{y}, \boldsymbol{b} k / \boldsymbol{\alpha}] \in A[\boldsymbol{c} / \boldsymbol{x}]^{*} \\
\quad \text { and } \boldsymbol{\lambda} k \cdot\left[\mathrm{p}_{2}\left(\left\langle\varepsilon^{\prime *}, \zeta^{*}\right\rangle\right)\right][\boldsymbol{c} / \boldsymbol{x}, \boldsymbol{a} k / \boldsymbol{y}, \boldsymbol{b} k / \boldsymbol{\alpha}] \in B[\boldsymbol{c} / \boldsymbol{x}]^{*} \\
\Leftrightarrow \boldsymbol{\lambda} k \cdot\left[\varepsilon^{\prime *}\right][\boldsymbol{c} / \boldsymbol{x}, \boldsymbol{a} k / \boldsymbol{y}, \boldsymbol{b} k / \boldsymbol{\alpha}] \in A[\boldsymbol{c} / \boldsymbol{x}]^{*} \\
\quad \text { and } \boldsymbol{\lambda} k \cdot\left[\zeta^{*}\right][\boldsymbol{c} / \boldsymbol{x}, \boldsymbol{a} k / \boldsymbol{y}, \boldsymbol{b} k / \boldsymbol{\alpha}] \in B[\boldsymbol{c} / \boldsymbol{x}]^{*}
\end{aligned}
$$

which is true by induction hypothesis.

$-\varepsilon=\frac{\frac{\varepsilon^{\prime}}{\Gamma \vdash A_{1} \wedge A_{2} \mid \Delta}}{\Gamma \vdash A_{i} \mid \Delta}(i=1,2)$ : let us take $i=1$ (the other case is similar).

The induction hypothesis gives us:

$$
\boldsymbol{\lambda} k \cdot\left[\varepsilon^{\prime *}\right][\boldsymbol{c} / \boldsymbol{x}, \boldsymbol{a} k / \boldsymbol{y}, \boldsymbol{b} k / \boldsymbol{\alpha}] \in\left|A_{1}[\boldsymbol{c} / \boldsymbol{x}] \wedge A_{2}[\boldsymbol{c} / \boldsymbol{x}]\right|
$$

so we get by lemma A.5:

$$
\boldsymbol{\lambda} k \cdot\left[\mathrm{p}_{1}(x)\right]\left[\left(\boldsymbol{\lambda} k^{\prime} \cdot\left[\varepsilon^{\prime *}\right]\left[\boldsymbol{c} / \boldsymbol{x}, \boldsymbol{a} k^{\prime} / \boldsymbol{y}, \boldsymbol{b} k^{\prime} / \boldsymbol{\alpha}\right]\right) k / x\right] \in\left|A_{1}[\boldsymbol{c} / \boldsymbol{x}]\right|
$$

but:

$$
\begin{aligned}
\boldsymbol{\lambda} k \cdot\left[\mathrm{p}_{1}(x)\right]\left[\left(\boldsymbol{\lambda} k^{\prime} \cdot\left[\varepsilon^{\prime *}\right]\left[\boldsymbol{c} / \boldsymbol{x}, \boldsymbol{a} k^{\prime} / \boldsymbol{y}, \boldsymbol{b} k^{\prime} / \boldsymbol{\alpha}\right]\right) k / x\right] \\
=\boldsymbol{\lambda} k \cdot\left[\mathrm{p}_{1}(x)\right]\left[\left[\varepsilon^{\prime *}\right][\boldsymbol{c} / \boldsymbol{x}, \boldsymbol{a} k / \boldsymbol{y}, \boldsymbol{b} k / \boldsymbol{\alpha}] / x\right] \\
=\boldsymbol{\lambda} k \cdot\left[\mathrm{p}_{1}\left(\varepsilon^{\prime *}\right)\right][\boldsymbol{c} / \boldsymbol{x}, \boldsymbol{a} k / \boldsymbol{y}, \boldsymbol{b} k / \boldsymbol{\alpha}]
\end{aligned}
$$

so we can conclude.

$$
\begin{gathered}
-\varepsilon=\frac{\frac{\varepsilon^{\prime}}{\Gamma \vdash A \mid \Delta}}{\Gamma \vdash \forall x^{\tau} A \mid \Delta}(x \notin \mathrm{FV}(\Gamma, \Delta)) \text { : by induction hypothesis we have: } \\
\forall c \in \tau^{\mathrm{t}}, \boldsymbol{\lambda} k \cdot\left[\varepsilon^{\prime *}\right][\boldsymbol{c} / \boldsymbol{x}, \boldsymbol{a} k / \boldsymbol{y}, \boldsymbol{b} k / \boldsymbol{\alpha}] \in|A[c / x, \boldsymbol{c} / \boldsymbol{x}]|
\end{gathered}
$$

First, since $x$ is not free in $A_{1}, \ldots, A_{n}, B_{1}, \ldots, B_{m}$ we have $\left|A_{i}[c / x, \boldsymbol{c} / \boldsymbol{x}]\right|=$ $\left|A_{i}[\boldsymbol{c} / \boldsymbol{x}]\right|$ and $\left\|B_{i}[c / x, \boldsymbol{c} / \boldsymbol{x}]\right\|=\left\|B_{i}[\boldsymbol{c} / \boldsymbol{x}]\right\|$, so we still have $a_{i} \in\left|A_{i}[\boldsymbol{c} / \boldsymbol{x}]\right|$ and $b_{i} \in\left\|B_{i}[\boldsymbol{c} / \boldsymbol{x}]\right\|$. Using lemma A.1, we have:

$$
\begin{aligned}
\boldsymbol{\lambda} k . & {\left[\lambda x . \varepsilon^{\prime *}\right][\boldsymbol{c} / \boldsymbol{x}, \boldsymbol{a} k / \boldsymbol{y}, \boldsymbol{b} k / \boldsymbol{\alpha}] \in\left|\forall x^{\tau} A[\boldsymbol{c} / \boldsymbol{x}]\right| } \\
& \Leftrightarrow \forall c \in \tau^{\mathrm{t}}, \boldsymbol{\lambda} k \cdot[z x]\left[\left(\boldsymbol{\lambda} k^{\prime} \cdot\left[\lambda x \cdot \varepsilon^{\prime *}\right]\left[\boldsymbol{c} / \boldsymbol{x}, \boldsymbol{a} k^{\prime} / \boldsymbol{y}, \boldsymbol{b} k^{\prime} / \boldsymbol{\alpha}\right]\right) k / z, c / x\right] \in|A[\boldsymbol{c} / \boldsymbol{x}, c / x]| \\
& \Leftrightarrow \forall c \in \tau^{\mathrm{t}}, \boldsymbol{\lambda} k \cdot[z x]\left[\left[\lambda x \cdot \varepsilon^{\prime *}\right][\boldsymbol{c} / \boldsymbol{x}, \boldsymbol{a} k / \boldsymbol{y}, \boldsymbol{b} k / \boldsymbol{\alpha}] / z, c / x\right] \in|A[\boldsymbol{c} / \boldsymbol{x}, c / x]| \\
& \Leftrightarrow \forall c \in \tau^{\mathrm{t}}, \boldsymbol{\lambda} k .\left[\left(\lambda x . \varepsilon^{\prime *}\right) x\right][\boldsymbol{c} / \boldsymbol{x}, \boldsymbol{a} k / \boldsymbol{y}, \boldsymbol{b} k / \boldsymbol{\alpha}, c / x] \in|A[\boldsymbol{c} / \boldsymbol{x}, c / x]|
\end{aligned}
$$

and we conclude by induction hypothesis. 
$-\varepsilon=\frac{\frac{\varepsilon^{\prime}}{\Gamma \vdash \forall x^{\tau} A \mid \Delta}}{\Gamma \vdash A\left[a^{\tau} / x\right] \mid \Delta}$ : by induction hypothesis we have:

$$
\boldsymbol{\lambda} k \cdot\left[\varepsilon^{\prime *}\right][\boldsymbol{c} / \boldsymbol{x}, \boldsymbol{a} k / \boldsymbol{y}, \boldsymbol{b} k / \boldsymbol{\alpha}] \in\left|\forall x^{\tau} A[\boldsymbol{c} / \boldsymbol{x}]\right|
$$

Using lemma A.1, we have:

$$
\forall e \in \tau^{\mathrm{t}}, \boldsymbol{\lambda} k \cdot[z x]\left[\left(\boldsymbol{\lambda} k^{\prime} \cdot\left[\varepsilon^{\prime *}\right]\left[\boldsymbol{c} / \boldsymbol{x}, \boldsymbol{a} k^{\prime} / \boldsymbol{y}, \boldsymbol{b} k^{\prime} / \boldsymbol{\alpha}\right]\right) k / z, e / x\right] \in|A[\boldsymbol{c} / \boldsymbol{x}, e / x]|
$$

so by taking $e=\left[a^{\dagger}\right][\boldsymbol{c} / \boldsymbol{x}]$ we have:

$$
\begin{aligned}
\boldsymbol{\lambda} k . & {[z x]\left[\left(\boldsymbol{\lambda} k^{\prime} \cdot\left[\varepsilon^{\prime *}\right]\left[\boldsymbol{c} / \boldsymbol{x}, \boldsymbol{a} k^{\prime} / \boldsymbol{y}, \boldsymbol{b} k^{\prime} / \boldsymbol{\alpha}\right]\right) k / z, e / x\right] } \\
& =\boldsymbol{\lambda} k \cdot[z x]\left[\left[\varepsilon^{\prime *}\right][\boldsymbol{c} / \boldsymbol{x}, \boldsymbol{a} k / \boldsymbol{y}, \boldsymbol{b} k / \boldsymbol{\alpha}] / z,\left[a^{\dagger}\right][\boldsymbol{c} / \boldsymbol{x}] / x\right] \\
& =\boldsymbol{\lambda} k \cdot\left[\varepsilon^{\prime *} x\right]\left[\boldsymbol{c} / \boldsymbol{x}, \boldsymbol{a} k / \boldsymbol{y}, \boldsymbol{b} k / \boldsymbol{\alpha},\left[a^{\dagger}\right][\boldsymbol{c} / \boldsymbol{x}] / x\right] \\
& =\boldsymbol{\lambda} k \cdot\left[\varepsilon^{\prime *} a^{\dagger}\right][\boldsymbol{c} / \boldsymbol{x}, \boldsymbol{a} k / \boldsymbol{y}, \boldsymbol{b} k / \boldsymbol{\alpha}]
\end{aligned}
$$

since on the other hand we have $\left|A\left[\boldsymbol{c} / \boldsymbol{x},\left[a^{\dagger}\right][\boldsymbol{c} / \boldsymbol{x}] / x\right]\right|=\left|A\left[a^{\tau} / x\right][\boldsymbol{c} / \boldsymbol{x}]\right|$ we obtain:

$$
\boldsymbol{\lambda} k \cdot\left[\varepsilon^{\prime *} a^{\dagger}\right][\boldsymbol{c} / \boldsymbol{x}, \boldsymbol{a} k / \boldsymbol{y}, \boldsymbol{b} k / \boldsymbol{\alpha}] \in\left|A\left[a^{\tau} / x\right][\boldsymbol{c} / \boldsymbol{x}]\right|
$$

$-\varepsilon=\frac{\frac{\varepsilon^{\prime}}{\Gamma \vdash A \mid \Delta, A}}{(\Gamma \vdash \Delta, A)}$ : we prove here that for any $c_{1} \in \tau_{1}{ }^{\mathrm{t}}, \ldots, c_{k} \in \tau_{k}{ }^{\mathrm{t}}, a_{1} \in$ $\left|A_{1}[\boldsymbol{c} / \boldsymbol{x}]\right|, \ldots, a_{n} \in\left|A_{n}[\boldsymbol{c} / \boldsymbol{x}]\right|, b_{1} \in\left\|B_{1}[\boldsymbol{c} / \boldsymbol{x}]\right\|, \ldots, b_{m} \in\left\|B_{m}[\boldsymbol{c} / \boldsymbol{x}]\right\|$ and $b \in\|A[\boldsymbol{c} / \boldsymbol{x}]\|$, we have:

$$
\boldsymbol{\lambda} k \cdot\left[[\alpha] \varepsilon^{\prime *}\right][\boldsymbol{c} / x, \boldsymbol{a} k / \boldsymbol{y}, \boldsymbol{b} k / \boldsymbol{\alpha}, b k / \alpha] \in \Perp
$$

where $[[\alpha] t]$ is $[t] \alpha$. The induction hypothesis gives:

$$
\boldsymbol{\lambda} k \cdot\left[\varepsilon^{\prime *}\right][\boldsymbol{c} / x, \boldsymbol{a} k / \boldsymbol{y}, \boldsymbol{b} k / \boldsymbol{\alpha}, b k / \alpha] \in|A[\boldsymbol{c} / \boldsymbol{x}]|
$$

and we have:

$$
\begin{aligned}
& \boldsymbol{\lambda} k \cdot\left[[\alpha] \varepsilon^{\prime *}\right][\boldsymbol{c} / x, \boldsymbol{a} k / \boldsymbol{y}, \boldsymbol{b} k / \boldsymbol{\alpha}, b k / \alpha] \\
&=\boldsymbol{\lambda} k \cdot\left(\left[\varepsilon^{*}\right] \alpha\right)[\boldsymbol{c} / x, \boldsymbol{a} k / \boldsymbol{y}, \boldsymbol{b} k / \boldsymbol{\alpha}, b k / \alpha] \\
&=\boldsymbol{\lambda} k \cdot\left(\left[\varepsilon^{* *}\right](b k)\right)[\boldsymbol{c} / x, \boldsymbol{a} k / \boldsymbol{y}, \boldsymbol{b} k / \boldsymbol{\alpha}, b k / \alpha] \\
&=\boldsymbol{\lambda} k \cdot\left[\varepsilon^{\prime *}\right][\boldsymbol{c} / x, \boldsymbol{a} k / \boldsymbol{y}, \boldsymbol{b} k / \boldsymbol{\alpha}, b k / \alpha](b k) \\
&=\boldsymbol{\lambda} k \cdot\left(\boldsymbol{\lambda} k^{\prime} \cdot\left[\varepsilon^{\prime *}\right]\left[\boldsymbol{c} / x, \boldsymbol{a} k^{\prime} / \boldsymbol{y}, \boldsymbol{b} k^{\prime} / \boldsymbol{\alpha}, b k^{\prime} / \alpha\right]\right) k(b k)
\end{aligned}
$$

which is in $\Perp$ using the induction hypothesis and the fact that $b \in\|A[\boldsymbol{c} / \boldsymbol{x}]\|$.

$-\varepsilon=\frac{\frac{\varepsilon^{\prime}}{(\Gamma \vdash \Delta, A)}}{\Gamma \vdash A \mid \Delta}$ : the previous point gives us:

$$
\forall b \in\|A[\boldsymbol{c} / \boldsymbol{x}]\|, \boldsymbol{\lambda} k \cdot\left[\varepsilon^{\prime *}\right][\boldsymbol{c} / x, \boldsymbol{a} k / \boldsymbol{y}, \boldsymbol{b} k / \boldsymbol{\alpha}, b k / \alpha] \in \Perp
$$

so if we define $[\mu \alpha . t]=\boldsymbol{\lambda} \alpha .[t]$ (so we still have $[\mu \alpha .[\beta] t]=\boldsymbol{\lambda} \alpha[t] \beta$ ), we prove:

$$
\boldsymbol{\lambda} k \cdot\left[\mu \alpha \cdot \varepsilon^{\prime *}\right][\boldsymbol{c} / x, \boldsymbol{a} k / \boldsymbol{y}, \boldsymbol{b} k / \boldsymbol{\alpha}] \in|A[\boldsymbol{c} / \boldsymbol{x}]|
$$


In order to do that we choose $b \in\|A[\boldsymbol{c} / \boldsymbol{x}]\|$ and we prove:

$$
\boldsymbol{\lambda} k .\left(\boldsymbol{\lambda} k^{\prime} \cdot\left[\mu \alpha \cdot \varepsilon^{\prime *}\right]\left[\boldsymbol{c} / x, \boldsymbol{a} k^{\prime} / \boldsymbol{y}, \boldsymbol{b} k^{\prime} / \boldsymbol{\alpha}\right]\right) k(b k) \in \Perp
$$

but since we have:

$$
\begin{aligned}
\boldsymbol{\lambda} k . & \left(\boldsymbol{\lambda} k^{\prime} \cdot\left[\mu \alpha \cdot{\varepsilon^{\prime *}}^{*}\right]\left[\boldsymbol{c} / x, \boldsymbol{a} k^{\prime} / \boldsymbol{y}, \boldsymbol{b} k^{\prime} / \boldsymbol{\alpha}\right]\right) k(b k) \\
& =\boldsymbol{\lambda} k \cdot\left[\mu \alpha \cdot \varepsilon^{\prime *}\right][\boldsymbol{c} / x, \boldsymbol{a} k / \boldsymbol{y}, \boldsymbol{b} k / \boldsymbol{\alpha}](b k) \\
& =\boldsymbol{\lambda} k \cdot\left[\mu \alpha \cdot \varepsilon^{\prime *}\right](b k)[\boldsymbol{c} / x, \boldsymbol{a} k / \boldsymbol{y}, \boldsymbol{b} k / \boldsymbol{\alpha}] \\
& =\boldsymbol{\lambda} k \cdot\left[\mu \alpha \cdot \varepsilon^{\prime *}\right] \alpha[\boldsymbol{c} / x, \boldsymbol{a} k / \boldsymbol{y}, \boldsymbol{b} k / \boldsymbol{\alpha}, b k / \alpha] \\
& =\boldsymbol{\lambda} k \cdot\left(\boldsymbol{\lambda} \alpha \cdot\left[\varepsilon^{\prime *}\right]\right) \alpha[\boldsymbol{c} / x, \boldsymbol{a} k / \boldsymbol{y}, \boldsymbol{b} k / \boldsymbol{\alpha}, b k / \alpha] \\
& =\boldsymbol{\lambda} k \cdot\left[\varepsilon^{\prime *}\right][\boldsymbol{c} / x, \boldsymbol{a} k / \boldsymbol{y}, \boldsymbol{b} k / \boldsymbol{\alpha}, b k / \alpha]
\end{aligned}
$$

which is in $\Perp$ by the previous point.

\section{B Computational Adequacy}

The correctness of the machine of Sect. 5 (i.e. reduction preserves semantics) can be proved as usual (see e.g. [18]). Note that since the model Fam $(\mathcal{G})$ is typed, this would involve typing rules for environments and stacks.

For extraction, we acutally only need to show Proposition 7.3:

(i) If $\vdash t: \iota \mid$ in $\Lambda_{\Psi}$, then for all $n \in \mathbb{N}$ we have $(t, \varepsilon, \star) \succ(\bar{n}, e, \star)$ if $[t]=[n]$.

(ii) Let $\vdash t: \iota \rightarrow \iota$ in $\Lambda_{\Psi}$. For all $n, m \in \mathbb{N}$, if $\lambda k \cdot[t]\langle[\bar{n}], k\rangle=[\bar{m}]$ then $(t \bar{n}, \varepsilon, \star) \succ(\bar{m}, e, \star)$.

We prove this property here.

We use the usual technique of logical relations, and deal with Bar-Recursion using the usual technique for the PCF fixpoint operator (see e.g. [2]). As suggested by the interpretation of the calculus in $\mathrm{R}^{\mathrm{Fam}(\mathcal{G})}$, and similarly to what we have done for realizability (see Sect. 6 and App A), our logical relations will be build by orthogonality.

Idealy, we would process as follows: We would fix a binary relation $\pi$ between $\left(\Lambda_{\Psi} \times \mathrm{E} \times \Pi\right)$ and (strategies on) $[\iota]$, and as usual assume that $\pi$ is closed under anti-evaluation, i.e.

$$
(t, e, \pi) \pi a \quad \Longrightarrow \quad\left(t^{\prime}, e^{\prime}, \pi^{\prime}\right) \succ(t, e, \pi) \quad \Longrightarrow \quad\left(t^{\prime}, e^{\prime}, \pi^{\prime}\right) \pi a
$$

and that for all $(t, e, \pi)$ we have

$$
(t, e, \pi) \Pi \perp_{[\iota]}
$$

We would then devise a relation $\mathcal{R}_{\tau} \subseteq \Pi \times \llbracket \tau \rrbracket$ for each simple type $\tau$, and obtain by orthogonality a relation $\mathcal{R}_{\tau}^{\pi} \subseteq \mathrm{C} \times[\tau]$. However, we face a similar problem as with realizability in Sect. 6: we want to observe termination at $[\iota]=\mathrm{R} \llbracket \iota \rrbracket$, which is not a basic type. Moreover, the only strategy on $\llbracket \iota \rrbracket$ is the empty strategy; and applying it to (the interpretation of) a numeral $[\bar{n}]$ gives the empty strategy 
on $\mathrm{R}$ (which is the only possible one since $\mathrm{R}$ is the arena with just one (initial) oponent move).

Hence, we preceed similarly as for realizability, and consider for each simple type $\tau$ relations $\mathcal{R}_{\tau} \subseteq \Pi \times(\llbracket \iota \rrbracket \rightarrow \llbracket \tau \rrbracket)$ and $\mathcal{R}_{\tau}^{\pi} \subseteq \mathrm{C} \times(\llbracket \iota \rrbracket \rightarrow \llbracket \tau \rrbracket)$. The main point, given by Lemma B.5, is that we can define $\mathcal{R}_{\iota}$ so that for all $n \in \mathbb{N}$, we have $((\bar{n}, e), \boldsymbol{\lambda} k \cdot[\bar{n}]) \in \mathcal{R}_{\iota}^{\pi}$.

\section{B.1 The Logical Relation}

Let $\pi$ be a binary relation between $\left(\Lambda_{\Psi} \times \mathrm{E} \times \Pi\right)$ and (strategies on) $[\iota]$. We assume that $\pi$ is closed under anti-evaluation, i.e.

$$
(t, e, \pi) \pi a \quad \Longrightarrow \quad\left(t^{\prime}, e^{\prime}, \pi^{\prime}\right) \succ(t, e, \pi) \quad \Longrightarrow \quad\left(t^{\prime}, e^{\prime}, \pi^{\prime}\right) \pi a
$$

and that for all $(t, e, \pi)$ we have

$$
(t, e, \pi) \pi \perp_{[\iota]}
$$

Recall from Section 4 that we use a simply-typed $\boldsymbol{\lambda}$-calculus with constants in $\operatorname{Fam}(\mathcal{G})$.

To each simple type $\tau$, we will associate two binary relations

$$
\mathcal{R}_{\tau} \subseteq \Pi \times(\llbracket \iota \rrbracket \rightarrow \llbracket \tau \rrbracket) \quad \text { and } \quad \mathcal{R}_{\tau}^{\pi} \subseteq \mathrm{C} \times(\llbracket \iota \rrbracket \rightarrow[\tau])
$$

First, given any $\mathcal{A} \subseteq \Pi \times(\llbracket \iota \rrbracket \rightarrow \llbracket \tau \rrbracket)$, we let $\mathcal{A}^{\pi} \subseteq \mathrm{C} \times(\llbracket \iota \rrbracket \rightarrow[\tau])$ be

$$
\{((t, e), a) \mid \forall(\pi, b) \in \mathcal{A},(t, e, \pi) \pi \boldsymbol{\lambda} k \cdot a k(b k)\}
$$

If moreover $\mathcal{B} \subseteq \Pi \times(\llbracket \iota \rrbracket \rightarrow \llbracket \sigma \rrbracket)$, we define $\left(\mathcal{A}^{\pi} \cdot \mathcal{B}\right) \subseteq \Pi \times(\llbracket \iota \rrbracket \rightarrow \llbracket \tau \rightarrow \sigma \rrbracket)$ as

$$
\left\{(\langle c, \pi\rangle, \boldsymbol{\lambda} k \cdot\langle a k, b k\rangle) \mid(c, a) \in \mathcal{A}^{\pi} \text { and }(\pi, b) \in \mathcal{B}\right\}
$$

For the moment, we assume given some $\mathcal{R}_{\iota} \subseteq \Pi \times(\llbracket \iota \rrbracket \rightarrow \llbracket \iota \rrbracket)$, and define $\mathcal{R}_{\tau}$ by induction on $\tau$ as follows:

$$
\begin{aligned}
\mathcal{R}_{\sigma \times \tau} & :=\left\{\left(\mathrm{kp}_{1}(\pi), \boldsymbol{\lambda} k \cdot \mathbf{i n}_{1}(a k)\right) \mid(\pi, a) \in \mathcal{R}_{\sigma}\right\} \\
& \cup\left\{\left(\mathrm{kp}_{2}(\pi), \boldsymbol{\lambda} k \cdot \mathbf{i n}_{2}(a k)\right) \mid(\pi, a) \in \mathcal{R}_{\tau}\right\} \\
\mathcal{R}_{\sigma \rightarrow \tau} & :=\left(\mathcal{R}_{\sigma}^{\pi} \cdot \mathcal{R}_{\tau}\right)
\end{aligned}
$$

This definition of $\mathcal{R}_{\tau}$ with $\mathcal{R}_{\iota}$ arbitrary is sufficient to deal with the $\lambda \mu$ calculus with products. The actual definition on $\mathcal{R}_{\iota}$ will be given in App. B.2, when discussing arithmetic constants.

\section{Theorem B.1 (Adequacy for the $\lambda \mu$-Calculus with Products). If}

$$
x_{1}: \tau_{1}, \ldots, x_{n}: \tau_{n} \vdash t: \tau \mid \alpha_{1}: \sigma_{1}, \ldots, \alpha_{m}: \sigma_{m}
$$

then for all

$$
\left(u_{1}, e_{1}\right) \mathcal{R}_{\tau_{1}}^{\pi} b_{1}, \ldots,\left(u_{n}, e_{n}\right) \mathcal{R}_{\tau_{n}}^{\pi} b_{n}
$$


and all

$$
\pi_{1} \mathcal{R}_{\sigma_{1}} a_{1}, \ldots, \pi_{m} \mathcal{R}_{\sigma_{m}} a_{m}
$$

we have

$$
(t, e) \mathcal{R}_{\tau}^{\Pi} \boldsymbol{\lambda} k . t(k)
$$

where

$$
e \quad:=\left(x_{1},\left(u_{1}, e_{1}\right)\right):: \cdots::\left(x_{n},\left(u_{n}, e_{n}\right)\right)::\left(\alpha_{1}, \pi_{1}\right):: \cdots::\left(\alpha_{m}, \pi_{m}\right):: \varepsilon
$$

and

$$
\boldsymbol{t}(k):=[t]\left[b_{1}(k) / x_{1}, \ldots, b_{n}(k) / x_{n}, a_{1}(k) / \alpha_{1}, \ldots, a_{m}(k) / \alpha_{m}\right]
$$

In particular, if $\vdash t: \tau \mid$, then we have $((t, \varepsilon), \boldsymbol{\lambda} k .[t]) \in \mathcal{R}_{\tau}^{\Pi}$.

Proof. By induction on typing judgments. In the following, we let $\Gamma$ be the context $x_{1}: \tau_{1}, \ldots, x_{n}: \tau_{n}$ and $\Delta$ be $\alpha_{1}: \sigma_{1}, \ldots, \alpha_{m}: \sigma_{m}$. Unless stated otherwise, we will always assume given $\left(u_{i}, e_{i}, b_{i}\right)_{1 \leq i \leq n}$ and $\left(\pi_{j}, a_{j}\right)_{1 \leq j \leq m}$ as in the statement of the theorem.

We reason by cases on the last applied typing rule.

$$
\overline{\Gamma, x_{0}: \tau \vdash x_{0}: \tau \mid \Delta}
$$

Let $\left(\left(u_{0}, e_{0}\right), b_{0}\right) \in \mathcal{R}_{\tau}^{\pi}$ and $(\pi, a) \in \mathcal{R}_{\tau}$. We have to show

$$
\left(\left(u_{0}, e_{0}\right), b_{0}\right) \pi \boldsymbol{\lambda} k . \boldsymbol{t}(k)(a k)
$$

We have

$$
(t, e, \pi) \succ\left(u_{0}, e_{0}, \pi\right)
$$

and

$$
\boldsymbol{\lambda} k . t(k)=\lambda k \cdot b_{0} k=b_{0}
$$

We are done since by assumption,

$$
\begin{gathered}
\left(u_{0}, e_{0}, \pi\right) \pi \lambda k . b_{0} k(a k) \\
\frac{\Gamma, x: \tau \vdash t: \sigma \mid \Delta}{\Gamma \vdash \lambda x . t: \tau \rightarrow \sigma \mid \Delta}
\end{gathered}
$$

Let $\left(\left(u_{0}, e_{0}\right), b_{0}\right) \in \mathcal{R}_{\tau}^{\pi}$ and $(\pi, a) \in \mathcal{R}_{\sigma}$. We have to show

$$
\left(\lambda x . t, e,\left\langle\left(u_{0}, e_{0}\right), \pi\right\rangle\right) \pi \boldsymbol{\lambda} k .\left(\boldsymbol{\lambda}\left\langle x, k^{\prime}\right\rangle . \boldsymbol{t}(k) k^{\prime}\right)\left\langle b_{0} k, a k\right\rangle
$$

We have

$$
\left(\lambda x . t, e,\left\langle\left(u_{0}, e_{0}\right), \pi\right\rangle\right) \succ\left(t,\left(\left(x,\left(u_{0}, e_{0}\right)\right):: e, \pi\right)\right.
$$

and

$$
\boldsymbol{\lambda} k .\left(\boldsymbol{\lambda}\left\langle x, k^{\prime}\right\rangle . \boldsymbol{t}(k) k^{\prime}\right)\left\langle b_{0} k, a k\right\rangle=\boldsymbol{\lambda} k . \boldsymbol{t}(k)\left[b_{0} k / x\right](a k)
$$


and we are donce since by induction hypothesis

$$
\begin{gathered}
\left(t,\left(x,\left(u_{0}, e_{0}\right)\right):: e, \pi\right) \pi \boldsymbol{\lambda} k \cdot \boldsymbol{t}(k)\left[b_{0} k / x\right](a k) \\
\frac{\Gamma \vdash t: \sigma \rightarrow \tau|\Delta \quad \Gamma \vdash u: \sigma| \Delta}{\Gamma \vdash t u: \tau \mid \Delta}
\end{gathered}
$$

Let $(\pi, a) \in \mathcal{R}_{\tau}$. We have to show

$$
(t u, e, \pi) \pi \boldsymbol{\lambda} k .\left(\boldsymbol{\lambda} k^{\prime} . \boldsymbol{t}(k)\left\langle\boldsymbol{u}(k), k^{\prime}\right\rangle\right)(a k)
$$

We have

$$
(t u, e, \pi) \quad \succ \quad(t, e,\langle(u, e), \pi\rangle)
$$

and

$$
\boldsymbol{\lambda} k .\left(\boldsymbol{\lambda} k^{\prime} \cdot \boldsymbol{t}(k)\left\langle\boldsymbol{u}(k), k^{\prime}\right\rangle\right)(a k)=\boldsymbol{\lambda} k \cdot \boldsymbol{t}(k)\langle\boldsymbol{u}(k), a k\rangle
$$

We are done, since on the one hand by induction hypothesis

$$
((t, e), \boldsymbol{\lambda} k . t(k)) \in \mathcal{R}_{\sigma \rightarrow \tau}^{\Pi}
$$

and on the other hand

$$
(\langle(u, e), \pi\rangle, \boldsymbol{\lambda} k \cdot\langle\boldsymbol{u}(k), a k\rangle) \in \mathcal{R}_{\sigma \rightarrow \tau}
$$

since by induction hypothesis

$$
\begin{gathered}
((u, e), \boldsymbol{\lambda} k \cdot \boldsymbol{u}(k)) \in \mathcal{R}_{\sigma}^{\pi} \\
\frac{\Gamma \vdash t_{1}: \tau_{1}\left|\Delta \quad \Gamma \vdash t_{2}: \tau_{2}\right| \Delta}{\Gamma \vdash\left\langle t_{1}, t_{2}\right\rangle: \tau_{1} \times \tau_{2} \mid \Delta}
\end{gathered}
$$

Let $(\pi, a) \in \mathcal{R}_{\tau_{i}}$. Hence $\left(\operatorname{kp}_{i}(\pi), \boldsymbol{\lambda} k . \mathbf{i n}_{i}(a k)\right) \in \mathcal{R}_{\tau_{1} \times \tau_{2}}$. We have to show

$$
\left(\left\langle t_{1}, t_{2}\right\rangle, e, \mathrm{kp}_{i}(\pi)\right) \pi \boldsymbol{\lambda} k \cdot\left(\boldsymbol{\lambda} k^{\prime} \text {.case } k^{\prime}\left\{\boldsymbol{t}_{1}(k), \boldsymbol{t}_{2}(k)\right\}\right)\left(\mathbf{i n}_{i}(a k)\right)
$$

We have

$$
\left(\left\langle t_{1}, t_{2}\right\rangle, e, \mathrm{kp}_{i}(\pi)\right) \succ\left(t_{i}, e, \pi\right)
$$

and

$$
\boldsymbol{\lambda} k \cdot\left(\boldsymbol{\lambda} k^{\prime} . \text { case } k^{\prime}\left\{\boldsymbol{t}_{1}(k), \boldsymbol{t}_{2}(k)\right\}\right)\left(\mathbf{i n}_{i}(a k)\right)=\boldsymbol{\lambda} k \cdot \boldsymbol{t}_{i}(k)(a k)
$$

and we are donce since by induction hypothesis

$$
\begin{gathered}
\left(\left(t_{i}, e\right), \boldsymbol{\lambda} k \cdot \boldsymbol{t}_{i}(k)\right) \in \mathcal{R}_{\tau_{i}}^{\Pi} \\
\frac{\Gamma \vdash t: \tau_{1} \times \tau_{2} \mid \Delta}{\Gamma \vdash \mathrm{p}_{i}(t): \tau_{i} \mid \Delta}(i=1,2)
\end{gathered}
$$

Let $(\pi, a) \in \mathcal{R}_{\tau_{i}}$. We have have to show

$$
\left(\mathrm{p}_{i}(t), e, \pi\right) \pi \boldsymbol{\lambda} k \cdot\left(\boldsymbol{\lambda} k^{\prime} \cdot \boldsymbol{t}(k)\left(\mathbf{i n}_{i} k^{\prime}\right)\right)(a k)
$$


We have

$$
\left(\mathrm{p}_{i}(t), e, \pi\right) \succ\left(t, e, \mathrm{kp}_{i}(\pi)\right)
$$

and we are done since

$$
\left(\mathrm{kp}_{i}(\pi), \boldsymbol{\lambda} k \cdot \mathbf{i n}_{i}(a k)\right) \in \mathcal{R}_{\tau_{1} \times \tau_{2}}
$$

and since by induction hypothesis

$$
\begin{gathered}
((t, e), \boldsymbol{\lambda} k . t(k)) \in \mathcal{R}_{\tau_{1} \times \tau_{2}}^{\Pi} \\
\frac{\Gamma \vdash t: \tau \mid \Delta, \alpha: \tau}{[\alpha] t:(\Gamma \vdash \Delta, \alpha: \tau)} \quad \frac{t:(\Gamma \vdash \Delta, \alpha: \tau)}{\Gamma \vdash \mu \alpha . t: \tau \mid \Delta}
\end{gathered}
$$

It is actually sufficent to consider the case of

$$
\frac{\frac{\Gamma \vdash t: \sigma \mid \Delta, \beta: \sigma}{\vdots}}{\Gamma \vdash \mu \alpha \cdot[\beta] t: \tau \mid(\Delta, \beta: \sigma) \backslash\{\alpha: \tau\}}
$$

Let $(\pi, a) \in \mathcal{R}_{\tau}$. Note that we can have either $\alpha=\beta$ (in which case $\tau=\sigma$ ) or $\alpha=\beta$. In both cases, by assumption we can assume given $\left(\pi^{\prime}, a^{\prime}\right) \in \mathcal{R}_{\sigma}$. We have to show

$$
(\mu \alpha .[\beta] t, e, \pi) \pi \boldsymbol{\lambda} k \cdot\left(\boldsymbol{\lambda} \alpha \cdot \boldsymbol{t}(k)\left(a^{\prime} k\right)\right)(a k)
$$

We have

$$
(\mu \alpha .[\beta] t, e, \pi) \quad \succ \quad\left(t,(\alpha, \pi):: e, \pi^{\prime}\right)
$$

and we are done since by induction hypothesis

$$
((t,(\alpha, \pi):: e), \boldsymbol{\lambda} k \cdot \boldsymbol{t}(k)[a k / \alpha]) \in \mathcal{R}_{\sigma}^{\Pi}
$$

\section{B.2 Adequacy for Arithmetical Constants}

From now on, we let $(t, e, \pi) \pi a$ iff either $a=\perp_{[\iota]}$ or $a=[\bar{n}]$ and $(t, e, \pi) \succ$ $\left(\bar{n}, e^{\prime}, \star\right)$ for some $n \in \mathbb{N}$ and $e^{\prime} \in \mathrm{E}$.

We now proceed to the definition of $\mathcal{R}_{\iota}$. It will be defined as a least fixpoint in the complete lattice $\mathcal{P}(\Pi \times(\llbracket \iota \rrbracket \rightarrow \llbracket \iota \rrbracket))$. Given $X \subseteq \Pi \times(\llbracket \iota \rrbracket \rightarrow \llbracket \iota \rrbracket)$, let $F(X) \subseteq \Pi \times(\llbracket \iota \rrbracket \rightarrow \llbracket \iota \rrbracket)$ be

$$
\begin{aligned}
& \{(\star, \lambda k . k)\} \\
\cup & \{(\operatorname{ksucc}(\pi), \boldsymbol{\lambda} k \cdot \widetilde{\operatorname{ksucc}}(a k)) \mid(\pi, a) \in X\} \\
\cup & \bigcup_{\mathcal{A} \subseteq \Pi \times(\llbracket \iota \rrbracket \rightarrow \llbracket \tau \rrbracket)}\{(\operatorname{krec}(u, v, e, \pi), \boldsymbol{\lambda} k \cdot \operatorname{krec}(b k)(c k)(a k)) \mid \\
& \left.((u, e), b) \in \mathcal{A}^{\mathbb{N}},((v, e), c) \in\left(X^{\pi} \cdot \mathcal{A}^{\pi} \cdot \mathcal{A}\right)^{\pi} \&(\pi, a) \in \mathcal{A}\right\}
\end{aligned}
$$


where

$$
\begin{aligned}
& \widetilde{\mathrm{ksucc}}:=\boldsymbol{\lambda} a \cdot \boldsymbol{\lambda} n \cdot a(\widetilde{\operatorname{succ}} n) \\
& \widetilde{\mathrm{krec}}:=\boldsymbol{\lambda} b \cdot \boldsymbol{\lambda} c \cdot \boldsymbol{\lambda} a \cdot \boldsymbol{\lambda} n \cdot \widetilde{\mathrm{rec}} b\left(\boldsymbol{\lambda} m . c^{\bullet}(\boldsymbol{\lambda} k . k m)\right) n a \\
& \text { where } c^{\bullet}:=\boldsymbol{\lambda} x \cdot \boldsymbol{\lambda} y \cdot \boldsymbol{\lambda} z . c\langle x, y, z\rangle
\end{aligned}
$$

Note that

$$
\begin{aligned}
\text { succ } & =\boldsymbol{\lambda} n \boldsymbol{\lambda} k \cdot n(\widetilde{\mathrm{ksucc}} k) \\
\text { rec } & =\boldsymbol{\lambda} u \cdot \boldsymbol{\lambda} v \cdot \boldsymbol{\lambda} n \cdot \boldsymbol{\lambda} k \cdot n(\widetilde{\mathrm{krec}} u v k)
\end{aligned}
$$

Lemma B.2. If $X \subseteq Y$ then $F(X) \subseteq F(Y)$.

Proof. Let $(\pi, a) \in F(X)$. We show $(c, a) \in F(Y)$ by cases on the form of $\pi$. If $\pi$ is of the form $\star$ or $\operatorname{ksucc}\left(\pi^{\prime}\right)$ then the result is trivial. The remaining case follows from the following (usual) observation: since $X \subseteq Y$ implies $Y^{\pi} \subseteq X^{\pi}$ we have $\left(Y^{\pi} \cdot \mathcal{A}^{\pi} \cdot \mathcal{A}\right) \subseteq\left(X^{\pi} \cdot \mathcal{A}^{\pi} \cdot \mathcal{A}\right)$ hence $\left(X^{\pi} \cdot \mathcal{A}^{\pi} \cdot \mathcal{A}\right)^{\pi} \subseteq\left(Y^{\pi} \cdot \mathcal{A}^{\pi} \cdot \mathcal{A}\right)^{\pi}$.

Using Tarski's fixpoint theorem, we let $\mathcal{R}_{\iota}$ be least fixed-point of $F$. Hence,

$$
\mathcal{R}_{\iota}=F^{\lambda}(\emptyset)
$$

for some ordinal $\lambda$. For ordinals $\alpha \leq \lambda$, let

$$
\mathcal{R}_{\iota}^{\alpha}:=F^{\alpha}(\emptyset)
$$

Note that

$$
\mathcal{R}_{\iota}^{\beta+1}=F\left(\mathcal{R}_{\iota}^{\beta}\right)
$$

In other words, $\mathcal{R}_{\iota}$ is the smallest subset of $\Pi \times(\llbracket \iota \rrbracket \rightarrow \llbracket \iota \rrbracket)$ such that

$-(\star, \lambda k . k) \in \mathcal{R}_{\iota}$,

- if $(\pi, a) \in \mathcal{R}_{\iota}$, then $(\operatorname{ksucc}(\pi), \lambda k \cdot \widetilde{\operatorname{ksucc}}(a k)) \in \mathcal{R}_{\iota}$, and

- if $\mathcal{A} \subseteq \Pi \times(\llbracket \iota \rrbracket \rightarrow \llbracket \tau \rrbracket)$, then for all $u, v, e, \pi$ and all $a, b, c$ such that $((u, e), b) \in \mathcal{A}^{\pi},((v, e), c) \in\left(\mathcal{R}_{\iota}^{\pi} \cdot \mathcal{A}^{\pi} \cdot \mathcal{A}\right)^{\pi}$, and $(\pi, a) \in \mathcal{A}$, we have

$$
(\operatorname{krec}(u, v, e, \pi), \lambda k \cdot \widetilde{\operatorname{krec}}(b k)(c k)(a k))) \in \mathcal{R}_{\iota}
$$

Lemma B.3. ((succ, $e), \boldsymbol{\lambda} k$. [succ $]) \in \mathcal{R}_{\iota \rightarrow \iota}^{\Pi}$

Proof. We have to show

$$
\text { (succ, } e, \pi) \pi \boldsymbol{\lambda} k \cdot \boldsymbol{\lambda}\langle n, y\rangle \cdot(\operatorname{succ} n y)(a k)
$$

for all $(\pi, a) \in \mathcal{R}_{\iota \rightarrow \iota}$. Since $\mathcal{R}_{\iota \rightarrow \iota}=\mathcal{R}_{\iota}^{\Pi} \cdot \mathcal{R}_{\iota}$, this amounts to show

$$
\text { (succ, } \left.e,\left\langle\left(t, e^{\prime}\right), \pi\right\rangle\right) \pi \boldsymbol{\lambda} k \cdot \operatorname{succ}(a k)(b k)
$$

for all $\left(\left(t, e^{\prime}\right), a\right) \in \mathcal{R}_{\iota}^{\Pi}$ and all $(\pi, b) \in \mathcal{R}_{\iota}$. But

$$
\text { (succ, } \left.e,\left\langle\left(t, e^{\prime}\right), \pi\right\rangle\right) \quad \succ \quad\left(t, e^{\prime}, \operatorname{ksucc}(\pi)\right)
$$

On the other hand,

$$
\begin{aligned}
\lambda k \cdot \operatorname{succ}(a k)(b k) & =\boldsymbol{\lambda} k \cdot a k(\boldsymbol{\lambda} n \cdot b k(\widetilde{\operatorname{succ}} n)) \\
& =\boldsymbol{\lambda} k \cdot(a k)(\operatorname{ksucc}(b k))
\end{aligned}
$$

and we are done since $\left(\left(t, e^{\prime}\right), a\right) \in \mathcal{R}_{\iota}^{\pi}$ and $(\operatorname{ksucc}(\pi), \boldsymbol{\lambda} k \widetilde{\operatorname{ksucc}}(b k)) \in \mathcal{R}_{\iota}$. 
Lemma B.4. Let $\tau \in \mathcal{T}, \mathcal{A} \subseteq \Pi \times(\llbracket \iota \rrbracket \rightarrow \llbracket \tau \rrbracket)$, and $\alpha \leq \lambda$. For all $u, v \in \Lambda, e \in$ $\mathrm{E}, b \in \llbracket \iota \rrbracket \rightarrow[\tau]$, and $c \in \llbracket \iota \rrbracket \rightarrow[\iota \rightarrow \tau \rightarrow \tau]$ such that $((u, e), b) \in \mathcal{A}^{\pi}$ and $((v, e), c) \in\left(\mathcal{R}_{\iota}^{\alpha \pi} \cdot \mathcal{A}^{\pi} \cdot \mathcal{A}\right)^{\pi}$, we have

$$
((\operatorname{rec}(u, v), e), \boldsymbol{\lambda} k \cdot \boldsymbol{\lambda}\langle n, y\rangle \cdot \mathbf{r e c}(b k)(c k) n y) \in\left(\mathcal{R}_{\iota}^{\alpha \pi} \cdot \mathcal{A}\right)^{\pi}
$$

In partcular, if $\mathcal{A}=\mathcal{R}_{\tau}$ and $\alpha=\lambda$, then we get

$$
((\operatorname{rec}(u, v), e), \boldsymbol{\lambda} k \cdot \boldsymbol{\lambda}\langle n, y\rangle \cdot \operatorname{rec}(b k)(c k) n y) \in \mathcal{R}_{\iota \rightarrow \tau}^{\pi}
$$

Proof. We have to show

$$
\left(\operatorname{rec}(u, v), e,\left\langle\left(t, e^{\prime}\right), \pi\right\rangle\right) \pi \boldsymbol{\lambda} k \cdot \operatorname{rec}(b k)(c k)(a k)(d k)
$$

for all $\left(\left(t, e^{\prime}\right), a\right) \in \mathcal{R}_{\iota}^{\alpha \pi}$ and all $(\pi, d) \in \mathcal{A}$. We have

$$
\left(\operatorname{rec}(u, v), e,\left\langle\left(t, e^{\prime}\right), \pi\right\rangle\right) \quad \succ \quad\left(t, e^{\prime}, \operatorname{krec}(u, v, e, \pi)\right)
$$

On the other hand,

$$
\begin{aligned}
\boldsymbol{\lambda} k \cdot \operatorname{rec}(b k)(c k)(a k)(d k) & =\boldsymbol{\lambda} k \cdot a k\left(\boldsymbol{\lambda} n \cdot \widetilde{\operatorname{rec}}(b k)\left(\boldsymbol{\lambda} y \cdot(c k)^{\bullet}\left(\boldsymbol{\lambda} k^{\prime} \cdot k^{\prime} y\right)\right) n(d k)\right) \\
& \quad \boldsymbol{w h e r e}(c k)^{\bullet}:=\boldsymbol{\lambda} x \cdot \boldsymbol{\lambda} y \cdot \boldsymbol{\lambda} z \cdot(c k)\langle x, y, z\rangle \\
& \boldsymbol{\lambda} k \cdot a k(\overline{\operatorname{krec}}(b k)(c k)(d k))
\end{aligned}
$$

We are done as $\left(\left(t, e^{\prime}\right), a\right) \in \mathcal{R}_{\iota}^{\pi}$ and $(\operatorname{krec}(u, v, e, \pi), \boldsymbol{\lambda} k \cdot \widetilde{\operatorname{krec}}(b k)(c k)(d k)) \in \mathcal{R}_{\iota}$.

Lemma B.5. For all $n \in \mathbb{N}$ and all $e \in \mathrm{E}$, we have $((\bar{n}, e), \boldsymbol{\lambda} k \cdot[\bar{n}]) \in \mathcal{R}_{\iota}^{\Pi}$.

Proof. We show by induction on ordinals $\alpha \leq \lambda$ that for all $(\pi, a) \in \mathcal{R}_{\iota}^{\alpha}$, we have $(\bar{n}, e, \pi) \pi \boldsymbol{\lambda} k \cdot[\bar{n}](a k)$ for all $n \in \mathbb{N}$ and all $e \in \mathrm{E}$.

First, if $\alpha$ is a limit ordinal, then

$$
\mathcal{R}_{\iota}^{\alpha}=\bigcup_{\beta<\alpha} \mathcal{R}_{\iota}^{\beta}
$$

Hence $(\pi, a) \in \mathcal{R}_{\iota}^{\alpha}$ iff $(\pi, a) \in \mathcal{R}_{\iota}^{\beta}$ for some $\beta<\alpha$ and the result follows directly by induction hypothesis.

Otherwise, $\alpha$ is either $\emptyset$ or a limit ordinal $\beta+1$. We reason by cases on $(\pi, a) \in \mathcal{R}_{\iota}^{\alpha}$.

- Case of $(\star, \lambda k . k)$. Given $n \in \mathbb{N}$ and $e \in \mathrm{E}$, we have to show

$$
(\bar{n}, e, \star) \Pi \boldsymbol{\lambda} k \cdot[\bar{n}]\left(\left(\boldsymbol{\lambda} k^{\prime} \cdot k^{\prime}\right) k\right)
$$

But we are done since

$$
\boldsymbol{\lambda} k \cdot[\bar{n}]\left(\left(\boldsymbol{\lambda} k^{\prime} \cdot k^{\prime}\right) k\right)=\boldsymbol{\lambda} k \cdot[\bar{n}] k=[\bar{n}]
$$


- Case of $(\operatorname{ksucc}(\pi), \boldsymbol{\lambda} k \cdot \widetilde{\operatorname{ksucc}}(a k))$. In this case we have $\alpha=\beta+1$ and $(\pi, a) \in$ $\mathcal{R}_{\iota}^{\beta}$. Given $n \in \mathbb{N}$ and $e \in \mathrm{E}$, we have to show

$$
(\bar{n}, e, \operatorname{ksucc}(\pi)) \pi \boldsymbol{\lambda} k \cdot[\bar{n}]\left(\left(\boldsymbol{\lambda} k^{\prime} \cdot \widetilde{\operatorname{ksucc}}\left(a k^{\prime}\right)\right) k\right)
$$

First, we have

$$
(\bar{n}, e, \operatorname{ksucc}(\pi)) \succ(\overline{n+1}, e, \pi)
$$

On the other hand

$$
\begin{aligned}
\boldsymbol{\lambda} k \cdot[\bar{n}]\left(\left(\boldsymbol{\lambda} k^{\prime} \cdot \widetilde{\operatorname{ksucc}}\left(a k^{\prime}\right)\right) k\right) & =\boldsymbol{\lambda} k \cdot[\bar{n}](\widetilde{\operatorname{ksucc}}(a k)) \\
& =\boldsymbol{\lambda} k \cdot\left(\boldsymbol{\lambda} k^{\prime} \cdot k^{\prime} \widetilde{n}\right)(\widetilde{\operatorname{ksucc}}(a k)) \\
& =\boldsymbol{\lambda} k \cdot \widetilde{\operatorname{kucc}}(a k) \widetilde{n} \\
& =\boldsymbol{\lambda} k \cdot(\boldsymbol{\lambda} a \cdot \boldsymbol{\lambda} n \cdot a(\widetilde{\operatorname{succ}} n))(a k) \widetilde{n} \\
& =\boldsymbol{\lambda} k \cdot a k(\widetilde{\operatorname{succ} \tilde{n})} \\
& =\boldsymbol{\lambda} k \cdot a k \tilde{n+1} \\
& =\boldsymbol{\lambda} k \cdot\left(\boldsymbol{\lambda} k^{\prime} \cdot k^{\prime} \widetilde{n+1}\right)(a k) \\
& =\boldsymbol{\lambda} k \cdot[\overline{n+1}](a k)
\end{aligned}
$$

Now we are done since by induction hypothesis, we have

$$
(\overline{n+1}, e, \pi) \pi \boldsymbol{\lambda} k \cdot[\overline{n+1}](a k)
$$

- Case of $(\operatorname{krec}(u, v, e, \pi), \boldsymbol{\lambda} k \cdot \widetilde{\operatorname{krec}}(b k)(c k)(a k))$. In this case we have $\alpha=\beta+1$ and $((u, e), b) \in \mathcal{A}^{\pi},((v, e), c) \in\left(\mathcal{R}_{\iota}^{\beta^{\pi}} \cdot \mathcal{A}^{\pi} \cdot \mathcal{A}\right)^{\pi}$, and $(\pi, a) \in \mathcal{A}$ for some $\mathcal{A} \subseteq \Pi \times(\llbracket \iota \rrbracket \rightarrow \llbracket \tau \rrbracket)$.

We have to show that for all $n \in \mathbb{N}$ and all $e^{\prime} \in \mathrm{E}$, we have

$$
\left(\bar{n}, e^{\prime}, \operatorname{krec}(u, v, e, \pi)\right) \pi \boldsymbol{\lambda} k \cdot[\bar{n}](\widetilde{\operatorname{krec}}(b k)(c k)(a k))
$$

We reason by cases on $n \in \mathbb{N}$.

- If $n=0$, then we have

$$
\left(\overline{0}, e^{\prime}, \operatorname{krec}(u, v, e, \pi)\right) \quad \succ \quad(u, e, \pi)
$$

On the other hand,

$$
\begin{aligned}
\boldsymbol{\lambda} k \cdot[\overline{0}](\widetilde{\operatorname{krec}}(b k)(c k)(a k))= & \boldsymbol{\lambda} k \cdot \widetilde{\operatorname{rec}}(b k)\left(\boldsymbol{\lambda} m \cdot(c k)^{\bullet}\left(\boldsymbol{\lambda} k^{\prime} \cdot k^{\prime} m\right)\right) \widetilde{0}(a k) \\
& \quad \text { where }(c k) \bullet:=\boldsymbol{\lambda} x \cdot \boldsymbol{\lambda} y \cdot \boldsymbol{\lambda} z \cdot c k\langle x, y, z\rangle \\
= & \boldsymbol{\lambda} k \cdot b k(a k)
\end{aligned}
$$

We are done since $((u, e), b) \in \mathcal{A}^{\pi}$ and $(\pi, a) \in \mathcal{A}$ by assumption.

- Otherwise, $n=m+1$. We have

$$
\left(\overline{m+1}, e^{\prime}, \operatorname{krec}(u, v, e, \pi)\right) \quad \succ \quad\left(v, e,\left\langle\left(\bar{m}, e^{\prime}\right),\langle(\operatorname{rec}(u, v) \bar{m}, e), \pi\rangle\right\rangle\right)
$$


On the other hand,

$$
\begin{aligned}
& \boldsymbol{\lambda} k \cdot[\overline{m+1}](\widetilde{\operatorname{krec}}(b k)(c k)(a k))=\boldsymbol{\lambda} k \cdot[\overline{m+1}]\left(\boldsymbol{\lambda} x \cdot \widetilde{\operatorname{rec}}(b k)\left(\boldsymbol{\lambda} m \cdot(c k)^{\bullet}\left(\boldsymbol{\lambda} k^{\prime} \cdot k^{\prime} m\right)\right) x(a k)\right) \\
&=\boldsymbol{\lambda} k \cdot \widetilde{\operatorname{rec}}(b k)\left(\boldsymbol{\lambda} m \cdot(c k)^{\bullet}\left(\boldsymbol{\lambda} k^{\prime} \cdot k^{\prime} m\right)\right)(m+1(a k) \\
&=\boldsymbol{\lambda} k \cdot(c k)^{\bullet}[\bar{m}]\left(\widetilde{\operatorname{rec}}(b k)\left(\boldsymbol{\lambda} m \cdot(c k)^{\bullet}\left(\boldsymbol{\lambda} k^{\prime} \cdot k^{\prime} m\right)\right) \widetilde{m}\right)(a k) \\
&=\boldsymbol{\lambda} k \cdot(c k)^{\bullet}[\bar{m}]\left(\boldsymbol{\lambda} y \cdot \widetilde{\operatorname{rec}}(b k)\left(\boldsymbol{\lambda} m \cdot(c k)^{\bullet}\left(\boldsymbol{\lambda} k^{\prime} \cdot k^{\prime} m\right)\right) \widetilde{m} y\right)(a k) \\
&=\boldsymbol{\lambda} k \cdot(c k)^{\bullet}[\bar{m}]\left(\boldsymbol{\lambda} y \cdot[\bar{m}]\left(\boldsymbol{\lambda} x \cdot \widetilde{\operatorname{rec}}(b k)\left(\boldsymbol{\lambda} m \cdot(c k)^{\bullet}\left(\boldsymbol{\lambda} k^{\prime} \cdot k^{\prime} m\right)\right) x y\right)\right)(a k) \\
&=\boldsymbol{\lambda} k \cdot(c k)\langle[\bar{m}],\langle\boldsymbol{\lambda} y \cdot \operatorname{rec}(b k)(c k)[\bar{m}] y, a k\rangle\rangle \\
& \text { where }(c k)^{\bullet}:=\boldsymbol{\lambda} x \cdot \boldsymbol{\lambda} y \cdot \boldsymbol{\lambda} z \cdot c k\langle x,\langle y, z\rangle\rangle
\end{aligned}
$$

Since $((v, e), c) \in\left(\mathcal{R}_{\iota}^{\beta^{\pi}} \cdot \mathcal{A}^{\pi} \cdot \mathcal{A}\right)^{\pi}$, we are done if

$\left(\left\langle\left(\bar{m}, e^{\prime}\right),\langle(\operatorname{rec}(u, v) \bar{m}, e), \pi\rangle\right\rangle, \boldsymbol{\lambda} k \cdot\langle[\bar{m}],\langle\boldsymbol{\lambda} y \cdot \operatorname{rec}(b k)(c k)[\bar{m}] y, a k\rangle\rangle\right) \in \mathcal{R}_{\iota}^{\beta^{\pi}} \cdot \mathcal{A}^{\pi} \cdot \mathcal{A}$

Now, by induction hypothesis we have $((\bar{m}, e), \boldsymbol{\lambda} k \cdot[\bar{m}]) \in \mathcal{R}_{\iota}^{\beta^{\pi}}$. We thus have to show

$$
(\langle(\operatorname{rec}(u, v) \bar{m}, e), \pi\rangle, \boldsymbol{\lambda} k \cdot\langle\boldsymbol{\lambda} y \cdot \operatorname{rec}(b k)(c k)[\bar{m}] y, a k\rangle) \in \mathcal{A}^{\pi} \cdot \mathcal{A}
$$

Since $(\pi, a) \in \mathcal{A}$, we are done if

$$
((\operatorname{rec}(u, v) \bar{m}, e), \boldsymbol{\lambda} k \cdot \boldsymbol{\lambda} y \cdot \operatorname{rec}(b k)(c k)[\bar{m}] y) \in \mathcal{A}^{\pi}
$$

Let $\left(\pi^{\prime}, a^{\prime}\right) \in \mathcal{A}$. Note that

$$
\left(\operatorname{rec}(u, v) \bar{m}, e, \pi^{\prime}\right) \quad \succ \quad\left(\operatorname{rec}(u, v), e,\left\langle(\bar{m}, e), \pi^{\prime}\right\rangle\right)
$$

On the other hand,

$$
\boldsymbol{\lambda} k \cdot(\boldsymbol{\lambda} y \cdot \boldsymbol{r e c}(b k)(c k)[\bar{m}] y)\left(a^{\prime} k\right)=\boldsymbol{\lambda} k \cdot(\boldsymbol{\lambda}\langle x, y\rangle \cdot \boldsymbol{r e c}(b k)(c k) x y)\left\langle[\bar{m}], a^{\prime} k\right\rangle
$$

Now, we are done since by induction hypothesis we have $((\bar{m}, e), \boldsymbol{\lambda} k \cdot[\bar{m}]) \in$ $\mathcal{R}_{\iota}^{\beta \pi}$, hence

$$
\left(\left\langle(\bar{m}, e), \pi^{\prime}\right\rangle, \lambda k \cdot\left\langle[\bar{m}], a^{\prime} k\right\rangle\right) \in \mathcal{R}_{\iota}^{\beta \pi} \cdot \mathcal{A}
$$

and by Lemma B.4:

$$
((\operatorname{rec}(u, v), e), \boldsymbol{\lambda} k \cdot \boldsymbol{\lambda}\langle x, y\rangle \cdot \operatorname{rec}(b k)(c k) x y) \in\left(\mathcal{R}_{\iota}^{\beta}{ }^{\pi} \cdot \mathcal{A}\right)^{\pi}
$$

It is now easy to extend computational adequacy (Thm. B.1) to the type system of the $\lambda \mu$-calculus with products extended with the typing rules for arithmetical constants of Section 5. We only detail the case of the recursor:

$$
\frac{\Gamma \vdash t: \tau|\Delta \quad \Gamma \vdash u: \iota \rightarrow \tau \rightarrow \tau| \Delta}{\Gamma \vdash \operatorname{rec}(t, u): \iota \rightarrow \tau \mid \Delta}
$$

Assuming the conventions used in the proof of Thm. B.1, we have to show

$$
\left.(\operatorname{rec}(t, u), e), \boldsymbol{\lambda} k \cdot \boldsymbol{\lambda}\left\langle n, k^{\prime}\right\rangle \cdot \operatorname{rec} \boldsymbol{t}(k) \boldsymbol{u}(k) n k^{\prime}\right) \in \mathcal{R}_{\iota \rightarrow \tau}^{\pi}
$$

This directly follows from Lemma B.4, since by hypothesis we have $((t, e), \boldsymbol{\lambda} k . \boldsymbol{t}(k)) \in$ $\mathcal{R}_{\tau}^{\Pi}$ and $((u, e), \boldsymbol{\lambda} k . \boldsymbol{u}(k)) \in \mathcal{R}_{\iota \rightarrow \tau \rightarrow \tau}^{\Pi}$. 


\section{B.3 Adequacy for Bar-Recursion}

We now discuss computational adequacy for the bar-recursor $\Psi$. We use the wellknown technique of fixpoint induction, as in the usual proofs of computational adequacy for PCF (see e.g. [2]).

We rely on the following remarks (for all type $\sigma$ ):

1. For all $(t, e)$ we have $\left((t, e), \perp_{\llbracket \iota \rrbracket \rightarrow[\sigma]}\right) \in \mathcal{R}_{\sigma}^{\pi}$.

Proof. Given $(\pi, a) \in \mathcal{R}_{\sigma}$, we have

$$
((t, e), \pi) \pi \boldsymbol{\lambda} k \cdot \perp_{\llbracket \iota \rrbracket \rightarrow[\sigma]} k(a k)
$$

since $\boldsymbol{\lambda} k . \perp_{\llbracket \iota \rrbracket \rightarrow[\sigma]} k(a k)=\perp_{[\iota]}$.

2. Let $(t, e)$ and let $\left(b_{m}\right)_{m \in \mathbb{N}} \in \llbracket \iota \rrbracket \rightarrow[\sigma]$ be a directed family such that $\left((t, e), b_{m}\right) \in \mathcal{R}_{\sigma}^{\Pi}$ for all $m \in \mathbb{N}$. Then $\left((t, e), \bigvee_{m \in \mathbb{N}} b_{m}\right) \in \mathcal{R}_{\sigma}^{\Pi}$.

Proof. Given $(\pi, a) \in \mathcal{R}_{\sigma}$, we have to show

$$
(t, e, \pi) \pi \boldsymbol{\lambda} k \cdot\left(\bigvee_{m \in \mathbb{N}} b_{m}\right) k(a k)
$$

We have

$$
\lambda k . \bigvee_{m \in \mathbb{N}}\left(b_{m} k(a k)\right)
$$

If $\boldsymbol{\lambda} k \cdot b_{m} k(a k)=\perp_{[\iota]}$ for all $m$, then $\boldsymbol{\lambda} k \cdot \bigvee_{m \in \mathbb{N}}\left(b_{m} k(a k)\right)=\perp_{[\iota]}$ and we are done.

Otherwise, there is some $m \in \mathbb{N}$ such that $\boldsymbol{\lambda} k \cdot b_{m} k(a k)=[\bar{n}]$ for some $n \in \mathbb{N}$, and we have $\boldsymbol{\lambda} k . \bigvee_{m \in \mathbb{N}}\left(b_{m} k(a k)\right)=[\bar{n}]$. But we are done since by assumption, $\boldsymbol{\lambda} k . b_{m} k(a k)=[\bar{n}]$ implies $(t, e, \pi) \succ\left(\bar{n}, e^{\prime}, \star\right)$.

We now fix $\tau \in \mathcal{T}, e \in \mathrm{E},((t, e), a) \in \mathcal{R}_{\iota \rightarrow(\tau \rightarrow \iota) \rightarrow \tau}^{\Pi}$ and $((u, e), b) \in \mathcal{R}_{(\iota \rightarrow \tau) \rightarrow \tau}^{\pi}$.

Given $c_{0}, \ldots, c_{n} \in[\tau]$, we let

$$
\widetilde{\Psi}_{\left\langle c_{0}, \ldots, c_{n}\right\rangle}^{m}:=\widetilde{\Psi}_{n+1}^{m}\left\langle c_{0}, \ldots, c_{n}\right\rangle
$$

We show that for all $m \in \mathbb{N}$, we have

$$
\left(\left(\Psi_{\tau}(t, u)\left\langle s_{0}, \ldots, s_{n}\right\rangle, e\right), \boldsymbol{\lambda} k \cdot \widetilde{\Psi}_{\left\langle a_{0} k, \ldots, a_{n} k\right\rangle}^{m}(a k)(b k)\right) \in \mathcal{R}_{\iota}^{\Pi}
$$

for all $n \in \mathbb{N}$ and all $\left(\left(s_{0}, e\right), b_{0}\right), \ldots,\left(\left(s_{n}, e\right), b_{n}\right) \in \mathcal{R}_{\tau}^{\pi}$.

We reason by induction on $m \in \mathbb{N}$. The base case $m=0$ follows from Rem (1) above.

For the induction step, first note that thanks to the results of App. B.1 and B.2, we have the adequacy for the construction $\langle\ldots .\rangle$.$@ :$

$$
\left(\left(\left\langle s_{0}, \ldots, s_{n}\right\rangle @ c, e\right), \lambda k \cdot\left\langle a_{0} k, \ldots, a_{n} k\right\rangle @(c k)\right) \in \mathcal{R}_{\iota \rightarrow \tau}^{\pi} \quad\left(((v, e), c) \in \mathcal{R}_{\iota \rightarrow \tau}^{\pi}\right)
$$


Let now $(\pi, d) \in \mathcal{R}_{\iota}$. We have to show:

$$
\left(\Psi_{\tau}(t, u)\left\langle s_{0}, \ldots, s_{n}\right\rangle, e, \pi\right) \pi \lambda k . \widetilde{\Psi}_{\left\langle a_{0} k, \ldots, a_{n} k\right\rangle}^{m+1}(a k)(b k)(d k)
$$

We have

$$
\begin{aligned}
& \left(\Psi_{\tau}(t, u)\left\langle s_{0}, \ldots, s_{n}\right\rangle, e, \pi\right) \succ \\
& \left(u, e,\left\langle\left(\left\langle s_{0}, \ldots, s_{n}\right\rangle @ \lambda_{-} . \operatorname{ex}_{\tau}\left(t \overline{n+1} \lambda x . \Psi_{\tau}(t, u)\left\langle s_{0}, \ldots, s_{n}, x\right\rangle\right), e\right), \pi\right\rangle\right)
\end{aligned}
$$

and $\boldsymbol{\lambda} k . \widetilde{\Psi}_{\left\langle a_{0} k, \ldots, a_{n} k\right\rangle}^{m+1}(a k)(b k)(d k)=$

$\boldsymbol{\lambda} k .(b k)\left\langle\left\langle a_{0} k, \ldots, a_{n} k\right\rangle @\right.$

$\left.\boldsymbol{\lambda}\left\langle_{-}, k_{0}\right\rangle \cdot\left[\operatorname{ex}_{\tau}\right]\left\langle\boldsymbol{\lambda} k_{1} \cdot(a k)\left\langle[\overline{n+1}], \boldsymbol{\lambda}\left\langle x, k_{2}\right\rangle . \widetilde{\Psi}_{\left\langle a_{0} k, \ldots, a_{n} k, x\right\rangle}^{m}(b k)(c k) k_{2}, k_{1}\right\rangle, k_{0}\right\rangle,(d k)\right\rangle$

Now we are done since by induction hypothesis:

$$
\left(\lambda x . \Psi_{\tau}(t, u)\left\langle s_{0}, \ldots, s_{n}, x\right\rangle, \lambda k . \lambda\left\langle x, k^{\prime}\right\rangle . \widetilde{\Psi}_{\left\langle a_{0} k, \ldots, a_{n} k, x\right\rangle}^{m} k^{\prime}\right) \in \mathcal{R}_{\tau \rightarrow \iota}^{\Pi}
$$

Using Rem (2) above we conclude:

Lemma B.6. Let $\tau \in \mathcal{T}, e \in \mathrm{E},((t, e), a) \in \mathcal{R}_{\iota \rightarrow(\tau \rightarrow \iota) \rightarrow \tau}^{\Pi}$ and $((u, e), b) \in$ $\mathcal{R}_{(\iota \rightarrow \tau) \rightarrow \tau}^{\prod}$.

Let moreover $n \in \mathbb{N}$ and $\left(\left(s_{0}, e\right), b_{0}\right), \ldots,\left(\left(s_{n}, e\right), b_{n}\right) \in \mathcal{R}_{\tau}^{\Pi}$.

We have:

$$
\left(\left(\Psi_{\tau}(t, u)\left\langle s_{0}, \ldots, s_{n}\right\rangle, e\right), \boldsymbol{\lambda} k . \widetilde{\Psi}_{\left\langle a_{0} k, \ldots, a_{n} k\right\rangle}^{\tau}(a k)(b k)\right) \in \mathcal{R}_{\iota}^{\Pi}
$$

\section{B.4 Proof of Proposition 7.3}

Corollary B.7. (i) If $\vdash t: \iota \mid$ in $\Lambda_{\Psi}$, then for all $n \in \mathbb{N}$ we have $(t, \varepsilon, \star) \succ$ $(\bar{n}, e, \star)$ if $[t]=[n]$.

(ii) Let $\vdash t: \iota \rightarrow \iota$ in $\Lambda_{\Psi}$. For all $n, m \in \mathbb{N}$, if $\boldsymbol{\lambda} k \cdot[t]\langle[\bar{n}], k\rangle=[\bar{m}]$ then $(t \bar{n}, \varepsilon, \star) \succ(\bar{m}, e, \star)$.

Proof. (i) By Thm. B.1, together with Lemmas B.3, B.4, B.5 and B.6, we have $(t, \varepsilon) \mathcal{R}_{\iota}^{\pi} \boldsymbol{\lambda} k .[n]$. Since $(\star, \boldsymbol{\lambda} k . k) \in \mathcal{R}_{\iota}$, it follows that

$$
(t, \varepsilon, \star) \pi \boldsymbol{\lambda} k \cdot[\bar{n}]\left(\left(\boldsymbol{\lambda} k^{\prime} \cdot k^{\prime}\right) k\right)
$$

and we are done by definition of $\pi$ since $\boldsymbol{\lambda} k \cdot[\bar{n}]\left(\left(\boldsymbol{\lambda} k^{\prime} \cdot k^{\prime}\right) k\right)=\boldsymbol{\lambda} k \cdot[\bar{n}] k=[\bar{n}]$ (note that the later holds even without using the $\eta$-rule, since $[\bar{n}]=\lambda k . k \widetilde{n}$ ).

(ii) Follows from (i) since for all $n \in \mathbb{N}$, we have $((\bar{n}, \varepsilon), \boldsymbol{\lambda} k \cdot[\bar{n}]) \in \mathcal{R}_{\iota}^{\Pi}$ by Lemma B.5. 


\section{Realization of Classical Choice Using Bar-Recursion}

In this Appendix, we prove Proposition 7.1. We recall its statement:

Proposition C.1. $\lambda k \cdot\left[t_{\mathrm{CAC}}^{\tau, A}\right] \in$

$\left|\forall x^{\iota}\left(\forall y^{\tau}(A \Rightarrow \perp) \Rightarrow \perp\right) \Rightarrow \forall f^{\iota \rightarrow \tau}\left(\forall x^{\iota} A[f x / y] \Rightarrow \perp\right) \Rightarrow \perp\right|$

Recall that

where

$$
\begin{aligned}
t_{\mathrm{CAC}}^{\tau, A} & =\lambda z \cdot \lambda c \cdot \Psi_{\tau \times A^{*}}\left(t_{\neg \neg \exists} z, \lambda a \cdot c\left(\lambda x \cdot \mathrm{p}_{1}(a x)\right)\left(\lambda x \cdot \mathrm{p}_{2}(a x)\right)\right)\langle\rangle \\
t_{\neg \neg \exists} & =\lambda a \cdot \lambda x \cdot \lambda k \cdot a x(\lambda y \cdot \lambda z \cdot k\langle y, z\rangle)
\end{aligned}
$$

The main point is to decompose the notion of realizability proposed in Sect. 6 w.r.t. the relativization of quantifications. It is convenient to extend the formulas defined in Sect. 2:

$$
A, B \quad:=\ldots\left|\tilde{\forall} x^{\tau} A \quad\right| \quad\left(\mathrm{r}_{\tau}\left(a^{\tau}\right) \times A\right) \Rightarrow B
$$

Hence, in extended formulas, the construction $\left(\mathrm{r}_{\tau}(a) \times A\right)$ is only allowed to appear to the left of an implication. The definition of realizability is extended as follows:

$$
\begin{aligned}
\left\|\tilde{\forall} x^{\tau} A\right\| & :=\bigcup_{a \in \tau^{\mathrm{\tau}}}\|A\|[a / x] \\
\left\|\left(\mathrm{r}_{\tau}(c) \times A\right) \Rightarrow B\right\| & :=\quad\left\{\boldsymbol{\lambda} k .\left\langle\boldsymbol{\lambda} k^{\prime} . \text { case } k^{\prime}\{c, a k\}, b k\right\rangle|a \in| A \mid \& b \in\|B\|\right\}
\end{aligned}
$$

Extended formulas and their realizability interpretation are inspired from ideas used in Krivine's Realizability [10]. We also extend the mapping (-) ${ }^{*}$ of Section 6, mapping extended formulas to simple types:

$$
\begin{aligned}
\left(\tilde{\forall} x^{\tau} A\right)^{*} & :=A^{*} \\
\left(\left(\mathrm{r}_{\tau}(a) \times A\right) \Rightarrow B\right)^{*} & :=\tau \times A^{*} \rightarrow B^{*}
\end{aligned}
$$

The following is the key for Proposition C.1. The argument is the usual one for bar-recursion, see e.g. [3, 4].

Lemma C.2. Let $B$ such that $B \Rightarrow \perp$ is an extended formula. Assume

$$
b \in\left|\forall x^{\iota}\left(\tilde{\forall} y^{\tau}(B \Rightarrow \perp) \Rightarrow \perp\right)\right| \quad \text { and } \quad c \in\left|\tilde{\forall} f^{\iota \rightarrow \tau}\left(\forall x^{\iota} B[f x / y] \Rightarrow \perp\right)\right|
$$

Then $\boldsymbol{\lambda} k . \widetilde{\Psi}_{\langle\rangle}^{B^{*}}(b k)(c k) \in|\perp|$.

Recall that we use notations (_- $)^{\bullet}$ and $\left({ }_{-}\right)^{\circ}$ for resp. currfication and uncurryfication. Recall also that the amount to which an expression is curryfied/uncurryfied depends on the context, and moreover that in $\mathcal{G},\left(_{-}\right)^{\bullet}$ and $\left({ }_{-}\right)^{\circ}$ are the identity.

Proof. First, note that for all extended formula $A$, we have $\operatorname{ex}_{A^{*}} \in|\tilde{\forall} \boldsymbol{x}(\perp \Rightarrow A)|$. This can be easily proved by induction on $A$. 
Recall that for all $a_{0}, \ldots, a_{n} \in\left[B^{*}\right]$, we have

$$
\begin{aligned}
\widetilde{\Psi}_{\left\langle a_{0}, \ldots, a_{n}\right\rangle}^{B^{*}}(b k)(c k)= & (c k)^{\bullet}\left(\left\langle a_{0}, \ldots, a_{n}\right\rangle @\right. \\
& \left.\boldsymbol{\lambda}_{-\cdot}\left[\operatorname{ex}_{B^{*}}\right]^{\bullet}\left((b k)^{\bullet}[\overline{n+1}]\left(\boldsymbol{\lambda} x . \widetilde{\Psi}_{\left\langle a_{0}, \ldots, a_{n}, x\right\rangle}^{B^{*}}(b k)(c k)\right)^{\circ}\right)\right)
\end{aligned}
$$

Assume now that $\boldsymbol{\lambda} k . \widetilde{\Psi}_{\langle\rangle}^{B^{*}}(b k)(c k) \notin|\perp|$. Since $\|\perp\|=\{\boldsymbol{\lambda} k . k\}$, this means that $\boldsymbol{\lambda} k . \widetilde{\Psi}_{\langle\rangle}^{B^{*}}(b k)(c k) k \notin \Perp$. By assumption on $c$, again since $\|\perp\|=\{\boldsymbol{\lambda} k . k\}$, this implies that there is $f \in(\iota \rightarrow \tau)^{\mathrm{t}}$ such that

$$
\boldsymbol{\lambda} k . \boldsymbol{\lambda}_{-}\left[\operatorname{ex}_{B^{*}}\right]^{\bullet}\left((b k)^{\bullet}[\overline{0}]\left(\boldsymbol{\lambda} x . \widetilde{\Psi}_{\langle x\rangle}^{B^{*}}(b k)(c k)\right)^{\circ}\right) \quad \notin \quad\left|\forall x^{\iota} B[f x / y]\right|
$$

By assumption on $b$, this implies that

$$
\lambda k \cdot \lambda\left\langle x, k^{\prime}\right\rangle . \widetilde{\Psi}_{\langle x\rangle}^{B^{*}}(b k)(c k) k^{\prime} \quad \notin|\tilde{\forall} y(B[[\overline{0}] / x] \Rightarrow \perp)|
$$

Hence, there is $d_{0} \in \tau^{\mathrm{t}}$ and $e_{0} \in \llbracket \iota \rrbracket \rightarrow\left[B^{*}\right]$ such that $e_{0} \in\left|B\left[[\overline{0}] / x, d_{0} / y\right]\right|$ and

$$
\boldsymbol{\lambda} k .\left(\boldsymbol{\lambda}\left\langle x, k^{\prime}\right\rangle . \widetilde{\Psi}_{\langle x\rangle}^{B^{*}}(b k)(c k) k^{\prime}\right)\left\langle e_{0} k,\left(\boldsymbol{\lambda} k^{\prime \prime} . k^{\prime \prime}\right) k\right\rangle \quad \notin \quad \Perp
$$

that is $\boldsymbol{\lambda} k \cdot \widetilde{\Psi}_{\left\langle e_{0} k\right\rangle}^{B^{*}}(b k)(c k) k \notin \Perp$.

By iterating the argument (using classical choice), we obtain a sequence $\left(d_{n}, e_{n}\right)_{n \in \mathbb{N}}$ such that for all $n \in \mathbb{N}$,

(i) $d_{n} \in \tau^{\mathrm{t}}$,

(ii) $e_{n} \in\left|B\left[[\bar{n}] / x, d_{n} / y\right]\right|$,

(iii) $\lambda k \cdot \widetilde{\Psi}_{\left\langle e_{0} k, \ldots, e_{n} k\right\rangle}^{B^{*}}(b k)(c k) k \notin \Perp$.

Let now $f \in[\iota \rightarrow \tau]$ be an innocent strategy such that $f[\bar{n}]=d_{n}$ for all $n \in \mathbb{N}$. Note that $f \in(\iota \rightarrow \tau)^{\mathrm{t}}$. Let moreover $g$ be such that $g[\bar{n}]=e_{n}$ for all $n \in \mathbb{N}$. It follows that

$$
h \quad:=\boldsymbol{\lambda} k \cdot \boldsymbol{\lambda}\left\langle x, k^{\prime}\right\rangle \cdot g^{\circ}\left\langle x, k^{\prime}\right\rangle k \in\left|\forall x^{\iota} B[f x / y]\right|
$$

By assumption on $c$, we have $\boldsymbol{\lambda} k \cdot \boldsymbol{\lambda} k^{\prime} . c k\left\langle h k, k^{\prime}\right\rangle \in|\perp|$. By continuity of $c$, this implies that there is $n \in \mathbb{N}$ such that

$$
\begin{aligned}
\boldsymbol{\lambda} k .(c k)^{\bullet} & \left(\left\langle e_{0} k, \ldots, e_{n} k\right\rangle @\right. \\
& \left.\boldsymbol{\lambda}_{-\cdot}\left[\operatorname{ex}_{B^{*}}\right]^{\bullet}\left((b k)^{\bullet}[\overline{n+1}]\left(\boldsymbol{\lambda} x . \widetilde{\Psi}_{\left\langle e_{0} k, \ldots, e_{n} k, x\right\rangle}^{B^{*}}(b k)(c k)\right)^{\circ}\right)\right) k \quad \in \quad \Perp
\end{aligned}
$$

It follows that

$$
\lambda k . \widetilde{\Psi}_{\left\langle e_{0} k, \ldots, e_{n} k\right\rangle}^{B^{*}}(b k)(c k) \quad \in \quad \Perp
$$

a contradiction.

We can now prove Proposition C.1. 
Proof (of Prop. C.1). Let $A$ be a formula. We have to show that $\lambda k$. $\left[t_{\text {CAC }}^{\tau, A}\right]$ realizes the formula

$$
\left|\forall x^{\iota}\left(\forall y^{\tau}(A \Rightarrow \perp) \Rightarrow \perp\right) \Rightarrow \forall f^{\iota \rightarrow \tau}\left(\forall x^{\iota} A[f x / y] \Rightarrow \perp\right) \Rightarrow \perp\right|
$$

We apply Lemma C.2 with $B:=\left(\mathrm{r}_{\tau}(y) \times A\right)$, and obtain that $\boldsymbol{\lambda} k . \widetilde{\Psi}_{\langle\rangle}^{\tau \times A^{*}}(b k)(c k) \in$ $|\perp|$ provided

$$
\begin{aligned}
& b \in\left|\forall x^{\iota}\left(\tilde{\forall} y^{\tau}\left(\left(\mathrm{r}_{\tau}(y) \times A\right) \Rightarrow \perp\right) \Rightarrow \perp\right)\right| \\
& \text { and } \quad c \in\left|\tilde{\forall} f^{\iota \rightarrow \tau}\left(\forall x^{\iota}\left(\mathrm{r}_{\tau}(f x) \times A[f x / y]\right) \Rightarrow \perp\right)\right|
\end{aligned}
$$

In order to conclude, it remains to show the two following points:

$$
\begin{aligned}
& \lambda k \cdot[\lambda a \cdot \lambda x \cdot \lambda b \cdot a x(\lambda y \cdot \lambda z \cdot b\langle y, z\rangle)] \\
&\left|\forall x^{\iota}\left(\forall y^{\tau}(A \Rightarrow \perp) \Rightarrow \perp\right) \Rightarrow \forall x^{\iota}\left(\tilde{\forall} y^{\tau}\left(\left(\mathrm{r}_{\tau}(y) \times A\right) \Rightarrow \perp\right) \Rightarrow \perp\right)\right|
\end{aligned}
$$

and

$\lambda k \cdot\left[\lambda c \cdot \lambda a . c\left(\lambda x \cdot \mathrm{p}_{1}(a x)\right)\left(\lambda x \cdot \mathrm{p}_{2}(a x)\right)\right] \in$

$$
\left|\forall f^{\iota \rightarrow \tau}\left(\forall x^{\iota} A[f x / y] \Rightarrow \perp\right) \Rightarrow \tilde{\forall} f^{\iota \rightarrow \tau}\left(\forall x^{\iota}\left(\mathrm{r}_{\tau}(f x) \times A[f x / y]\right) \Rightarrow \perp\right)\right|
$$

1. Let $a \in\left|\forall x^{\iota}\left(\forall y^{\tau}(A \Rightarrow \perp) \Rightarrow \perp\right)\right|, n \in \mathbb{N}$ and $b \in\left|\tilde{\forall} y^{\tau}\left(\left(\mathbf{r}_{\tau}(y) \times A\right) \Rightarrow \perp\right)\right|$. We have to show that

$$
\lambda k \cdot[\lambda a . \lambda x \cdot \lambda b . a x(\lambda y . \lambda z \cdot b\langle y, z\rangle)]\langle a k,[\bar{n}], b k, k\rangle \in \Perp
$$

i.e. that

$$
\boldsymbol{\lambda} k . a k\left\langle[\bar{n}], \boldsymbol{\lambda}\left\langle y, z, k^{\prime}\right\rangle . b k\left\langle\boldsymbol{\lambda} k^{\prime \prime} \text {.case } k^{\prime \prime}\{y, z\}, k^{\prime}\right\rangle, k\right\rangle \in \Perp
$$

Hence we are done if

$$
\boldsymbol{\lambda} k . \boldsymbol{\lambda}\left\langle y, z, k^{\prime}\right\rangle . b k\left\langle\boldsymbol{\lambda} k^{\prime \prime} \text {.case } k^{\prime \prime}\{y, z\}, k^{\prime}\right\rangle \in\left|\forall y^{\tau}(A \Rightarrow \perp)\right|
$$

But if $c \in \tau^{\mathrm{t}}$ and $d \in|A|$, we have

$$
\boldsymbol{\lambda} k .\left\langle\boldsymbol{\lambda} k^{\prime} \text {.case } k^{\prime}\{c, d k\}, k\right\rangle \in\left\|\left(\mathrm{r}_{\tau}(c) \times A\right) \Rightarrow \perp\right\|
$$

and we are done by assumption on $b$.

2. The proof is similar to that of (1). 


\section{Table of Contents}

On Bar Recursion and Choice in a Classical Setting .............. 1 Valentin Blot and Colin Riba 